\title{
Phase Stability of the Tin monochalcogenides SnS and SnSe: a Quasi-Harmonic Lattice-Dynamics
}

\author{
Study
}

\author{
Ioanna Pallikara ${ }^{1}$ and Jonathan M. Skelton ${ }^{1 *}$ \\ ${ }^{1}$ Department of Chemistry, University of Manchester, Oxford Road, Manchester M13 9PL, UK
}

\section{ABSTRACT}

The tin monochalcogenides $\mathrm{SnS}$ and SnSe adopt four different crystal structures, viz. orthorhombic Pnma and $\mathrm{Cmcm}$ and cubic rocksalt and $\pi$-cubic $\left(P 2{ }_{1} 3\right)$ phases, each of which has optimal properties for a range of potential applications. This rich phase space makes it challenging to identify the conditions under which the different phases are obtained. We have performed firstprinciples quasi-harmonic lattice-dynamics calculations to assess the relative stabilities of the four phases of $\mathrm{SnS}$ and $\mathrm{SnSe}$. We investigate dynamical stability through the presence or absence of imaginary modes in the phonon dispersion curves, and we compute Helmholtz and Gibbs free energies to evaluate the thermodynamic stability. We also consider applied pressures up to $15 \mathrm{GPa}$ to obtain simulated temperature-pressure phase diagrams. Finally, the relationships between the orthorhombic crystal phases are investigated by explicitly mapping the potential-energy surfaces along the imaginary phonon modes in the $\mathrm{Cmcm}$ phase, and the relationships between the cubic phases by transition-state modelling using the climbing-image nudged elastic-band method. 


\section{INTRODUCTION}

The binary chalcogenides $\mathrm{SnS}$ and $\mathrm{SnSe}$ have been of continuous scientific interest in recent years due to their wide range of applications in next-generation optoelectronic systems. ${ }^{1,2}$ Their semiconducting nature and outstanding electronic properties ${ }^{1-8}$ give rise to potential applications in solar cells, nanowires, superconductors and rechargeable batteries, ${ }^{9-13}$ and they are considered strong candidates for use in sustainable energy technologies as they are formed of nontoxic and earth-abundant elements. ${ }^{14-16}$

There are five reported crystal phases for $\mathrm{SnS}$ and $\mathrm{SnSe}$ in the recent literature, viz. orthorhombic Pnma and Cmcm structures and cubic $F m \overline{3} m$ (rocksalt), $P 2_{1} 3$ ( $\pi$-cubic) and $F \overline{4} 3 m$ (zincblende) structures. The Pnma phase is the ground-state structure of both materials and transitions to the $\mathrm{Cmcm}$ phase at elevated temperature. ${ }^{1,2}$ The $\pi$-cubic structure was recently reported as a metastable phase of both materials. ${ }^{17-19}$ There has been a substantial debate in the literature over the zincblende and rocksalt phases of $\mathrm{SnS}$, but zincblende $\mathrm{SnS}$ has been shown to be a high-energy, dynamically-unstable phase, ${ }^{14,20}$ and reports of zincblende SnS were likely misassignments of the recently-discovered $\pi$-cubic phase. ${ }^{18,21}$ Rocksalt $\mathrm{SnS}$ can be stabilized under certain conditions such as epitaxial growth, ${ }^{20,22}$ whereas rocksalt $\mathrm{SnSe}$ is a dynamically stable phase under ambient conditions. ${ }^{23} \mathrm{We}$ therefore focus on the Pnma, Cmcm, rocksalt and $\pi$ cubic phases, the structures of which are illustrated in Figure 1.

Among the most important applications of the tin monochalcogenides is in optoelectronic devices. SnS and SnSe are natural p-type semiconductors and promising absorber materials for photovoltaic (PV) devices. ${ }^{1,6} \mathrm{PV}$ materials are a significant contemporary research area due to the global demand for clean energy and ever-decreasing reserves of fossil fuels. ${ }^{14}$ Pnma SnS has a direct optical band gap between 1.3-1.43 eV, depending on the preparation method, and an indirect 
bandgap of $1.07 \mathrm{eV},{ }^{14}$ which are within the Shockley-Queisser limit for maximum PV efficiency. ${ }^{14}$ Pnma SnS also has an optical absorption coefficient of $10^{4}-10^{5} \mathrm{~cm}^{-1}$, which is larger than CdTe and many other established PV materials, ${ }^{14,24}$ together with a relatively high intrinsic free carrier concentration of $10^{17} \mathrm{~cm}^{-3} \cdot{ }^{25}$ Combined, these properties could lead to theoretical PV efficiencies of up to $24 \%,{ }^{25}$ which are large for thin-film solar cells, although there are some notable barriers to achieving this in practice. ${ }^{14,26}$ Pnma SnSe exhibits similar properties, with a direct a band gap of $0.9 \mathrm{eV},{ }^{27}$ an optical absorption coefficient around $10^{5} \mathrm{~cm}^{-1}$ in the visible region, and a similar intrinsic free carrier concentration around $10^{17} \mathrm{~cm}^{-3} \cdot 1,27$

(a) $\mathrm{SnSe}($ Pnma)

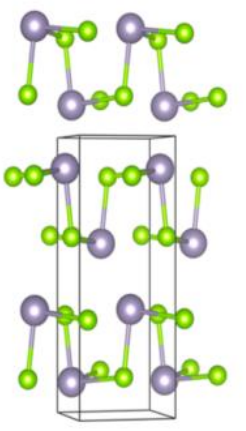

(b) $\mathrm{SnSe}(\mathrm{Cmcm})$

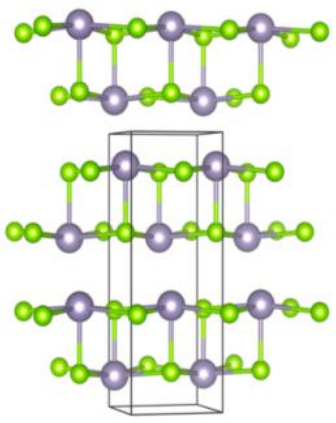

(c) SnSe (Rocksalt)

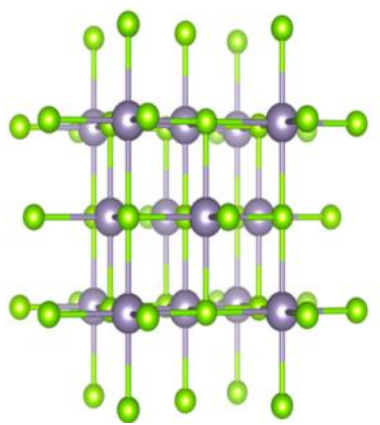

(d) $\mathrm{SnSe}(\pi$-cubic)

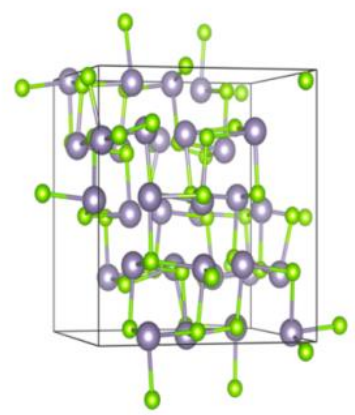

Figure 1. Representative structures of Pnma (a), Cmcm (b), rocksalt (c) and $\pi$-cubic SnSe (d). The images were produced using the VESTA software. ${ }^{28}$ The corresponding phases of SnS are isostructural and are shown in Figure S1 (Supporting Information).

The $\pi$-cubic polymorphs of $\mathrm{SnS}$ and $\mathrm{SnSe}$ also have electronic and optical properties that make them suitable for use in PV devices. ${ }^{1,18}$ The optical band gap of $\pi$-SnS is in the range of 1.66$1.72 \mathrm{eV}$ and its electrical conductivity is in the range of $10^{-6} \mathrm{~S} \mathrm{~cm}^{-1},{ }^{29}$ while $\pi$-SnSe has an optical band gap of approximately $1.4 \mathrm{eV}$ and an electrical conductivity around $5 \times 10^{-5} \mathrm{~S} \mathrm{~cm}^{-1}$. 
Another widely-studied potential application of the tin monochalcogenides is as thermoelectric materials. SnSe is one of the current champion thermoelectrics due to its high thermoelectric figure of merit $Z T$ of $2.6 \pm 0.3$ at $923 \mathrm{~K}$ along the crystallographic $b$-axis. Given that a thermoelectric system with a $Z T$ above 3 will exceed the efficiency of a traditional heat engine, ${ }^{30,31}$ this is to be considered outstanding. The high $Z T$ is partly due to the ultralow thermal conductivity associated with the anisotropy in the orthorhombic structures, which leads to strong phonon anharmonicity and large Grüneisen parameters. ${ }^{1}$ Indeed, both $\mathrm{SnS}$ and SnSe have ultralow reported thermal conductivities of $0.46 \mathrm{~W} \mathrm{~m}^{-1} \mathrm{~K}^{-1}$ and $0.23 \pm 0.3 \mathrm{~W} \mathrm{~m}^{-1} \mathrm{~K}^{-1}$ at $T=873$ and 973 $\mathrm{K}$, respectively. ${ }^{31} \mathrm{SnS}$ has also been explored as a potential thermoelectric material, but has so far only achieved a $Z T$ of 0.4 at $873 \mathrm{~K} .{ }^{31}$ The thermoelectric efficiency of both materials can however be further optimized, for example by chemical doping. ${ }^{1,32}$ It has been shown that the hightemperature phase transition from the Pnma to $\mathrm{Cmcm}$ phases alters the sample density and leads to enhanced phonon scattering, further lowering the thermal conductivity. ${ }^{33}$ The indirect band gap also decreases through the phase transition to $0.42 \mathrm{eV}$ in $\mathrm{SnS}$ and $0.39 \mathrm{eV}$ in SnSe. ${ }^{1,33}$ Finally, a recent study on SnSe revealed that the rocksalt phase has an even lower lattice thermal conductivity than the orthorhombic phase, suggesting this as a potential high-performance thermoelectric. ${ }^{34}$

As well as their applications to primary renewable energy generation, the tin monochalcogenides have also found uses in energy storage. Materials for high-performance rechargeable batteries have drawn considerable scientific interest lately, and one of the main focusses has been optimizing sodium-ion battery (SIB) chemistry. The carbonaceous anode materials typically used in lithium-ion batteries (LIBs) are not suitable for SIBs due the large volume changes during $\mathrm{Na}^{+}$ion extraction and insertion, ${ }^{35}$ but studies using $\mathrm{SnS} / \mathrm{SnSe}$ have demonstrated promising enhancements in surface area-to-volume ratio and improved electron 
transport, ${ }^{35}$ and SnSe composites with carbon, graphene oxide and carbon nanofiber matrices have shown promise in both LIBs and SIBs. ${ }^{1,35}$ The monochalcogenides may also have potential applications as supercapacitors: ${ }^{36}$ in 2014 Wang et al. demonstrated that spray-painted SnSe nanocrystals retained a capacity of $676 \mathrm{mAh} \mathrm{g}^{-1}$ after 80 cycles at a current density of $200 \mathrm{~mA} \mathrm{~g}^{-}$ ${ }^{1,1,37}$ while in 2017 Patil et al. reported that SnS thin films deposited using a low-cost chemical bath route had a maximum specific capacitance of $1375 \mathrm{~F} \mathrm{~g} \mathrm{~g}^{-1}$, an energy density of $60 \mathrm{Wh} \mathrm{kg}^{-1}$, and a power density of $8 \mathrm{KW} \mathrm{kg}^{-1} \cdot 38$

Finally, in addition to their numerous applications as energy materials, the tin monochalcogenides collectively also display a range of other interesting physical phenomena. Topological crystalline insulators (TCIs) have an insulating bulk and conducting surface states that are protected by both time-reversal symmetry and crystalline symmetries ${ }^{39}$ and are independent of spin-orbit interactions. ${ }^{1}$ Pnma $\mathrm{SnS}$ and SnSe both show weak spin-obit interactions and hence could possibly function as TCIs, ${ }^{1}$ while the rocksalt phases have also been proposed as native TCIs without the need for compression or alloying. ${ }^{40}$ The $P 2{ }_{1} 3$ spacegroup of the $\pi$-cubic structure is non-centrosymmetric, and $\pi$-SnS/SnSe are examples of a limited range of compounds whose piezoelectric moduli do not vanish and are chiral (optically active) and thus display secondharmonic generation. ${ }^{18}$

Despite considerable progress in recent years, widespread applications of these materials require some key challenges to be addressed. The existence of several different metastable phases leads to uncertainty over which phases are obtained under different synthesis conditions. Phase impurities have been shown to affect both $\mathrm{SnS}$ and SnSe-based PV devices as well as devices based on more complex multi-component semiconductors containing Sn and S/Se, where tin chalcogenides often form as impurities during synthesis. ${ }^{1,20}$ Such impurities can also reduce the 
thermal performance and affect electrical properties such as the Seebeck coefficient and electrical conductivity. ${ }^{1}$ A better fundamental understanding of the phase stability is thus essential to overcoming these obstacles. ${ }^{9}$

Various studies have investigated the structural properties and phase transitions in a subset of the tin monochalcogenides. We previously used harmonic lattice-dynamics calculations to investigate the $\mathrm{Sn}_{x} \mathrm{~S}_{y}$ phase space including the five reported monochalcogenide phases. ${ }^{20}$ The nature of the high-temperature $\mathrm{Cmcm}$ phase and its links to the ultralow thermal conductivity have been extensively explored. ${ }^{41-43}$ There are also various examples of studies investigating the effect of pressure on the monochalcogenides. For example, Yang et al. investigated the influence of pressure on the phase transition and the electronic and thermoelectric properties of $\mathrm{SnSe},{ }^{44}$ and Pal et al. performed Raman measurements and first-principles modelling to investigate pressureinduced phase transitions between the Pnma and $\mathrm{Cmcm}$ phases. ${ }^{45}$ Similarly, Liu et al. employed first-principles modelling to investigate the structural, thermodynamic, elastic and electronic properties of Pnma SnS at high pressure. ${ }^{46}$ However, most studies have focused on the orthorhombic phases of $\mathrm{SnS}$ or $\mathrm{SnSe}$, and there has not yet been a comprehensive study covering all four monochalcogenide phases of both materials.

In this work, we present a systematic theoretical investigation of the energetic and dynamical stability of the Pnma, Cmcm, rocksalt and $\pi$-cubic phases of SnS and SnSe using firstprinciples quasi-harmonic lattice-dynamics calculations. Using the quasi-harmonic approximation (QHA), we assess the dynamical stability of the different monochalcogenide phases through the presence or absence of imaginary modes in the phonon dispersion, and we use the harmonic Helmholtz and quasi-harmonic Gibbs free energies to compare the energetic stability as a function of temperature and pressure. We also establish the structural relationships between the 
orthorhombic phases by mapping the structural potential-energy surfaces (PESs) spanned by imaginary harmonic modes in the high-temperature $\mathrm{Cmcm}$ phases, and between the cubic phases by transition-state searching. This comprehensive characterization of the tin monochalcogenide phase space provides a reference point for future experimental work to obtain high-quality, phasepure samples, thereby helping to realize the many potential applications of these systems.

\section{AB INITIO LATTICE DYNAMICS CALCULATIONS}

\section{a. The Harmonic Approximation}

The theory of lattice dynamics provides a framework for modelling the lattice vibrations (phonons) in solids. Using the harmonic approximation, the second-order interatomic forceconstant matrices $\phi\left(l \kappa, l^{\prime} \kappa^{\prime}\right)$ are calculated as:

$$
\phi_{\alpha \beta}\left(l \kappa, l^{\prime} \kappa^{\prime}\right)=-\frac{\partial F_{\alpha}(l \kappa)}{\partial u_{\beta}\left(l^{\prime} \kappa^{\prime}\right)}=\frac{\partial^{2} \varphi}{\partial u_{\alpha}(l \kappa) \partial u_{\beta}\left(l^{\prime} \kappa^{\prime}\right)}
$$

where $\varphi$ is the potential energy of the crystal, $u_{\alpha}(l \kappa)=r_{\alpha}(l \kappa)-r_{\alpha, 0}(l \kappa)$ is the displacement of the $\kappa$ th atom in the $l$ th unit cell from its equilibrium position, $F_{\alpha}(l \kappa)$ is the corresponding restoring force, and the subscripts $\alpha$ and $\beta$ label the Cartesian directions.

Application of the Bloch theorem yields the dynamical matrix $\boldsymbol{D}(\boldsymbol{q})$, which after diagonalization give the $3 n_{a}$ phonon frequencies $\omega(\boldsymbol{q}, j)$ and corresponding displacement vectors $\boldsymbol{W}(\boldsymbol{q}, j)$ at the phonon wavevector $\boldsymbol{q}:{ }^{47,48}$

$$
\boldsymbol{D}(\boldsymbol{q})_{\kappa \kappa^{\prime}}^{\alpha \beta}=\sum_{l^{\prime}} \frac{1}{\sqrt{m_{\kappa} m_{\kappa^{\prime}}}} \phi_{\alpha \beta}\left(0 \kappa, l^{\prime} \kappa^{\prime}\right) e^{i \boldsymbol{k} \cdot\left[\boldsymbol{r}\left(l^{\prime} \kappa^{\prime}\right)-r\left(0 \kappa^{\prime}\right)\right]}
$$

The resulting phonon frequencies can then be used to evaluate the phonon density of states (DoS) $g(\omega)$ and phonon dispersion curves (band structures). 
The thermodynamic partition function $Z$ is related to the phonon frequencies via:

$$
Z(T)=\exp \left(-U_{L} / k_{B} T\right) \times \prod_{\boldsymbol{q} j} \frac{\exp \left(-\hbar \omega(\boldsymbol{q}, j) / 2 k_{B} T\right)}{1-\exp \left(-\hbar \omega(\boldsymbol{q}, j) / k_{B} T\right)}
$$

where $U_{L}$ is the lattice internal energy, equivalent to the $\varphi$ in Eq. 1, and the product runs over wavevectors $\boldsymbol{q}$ and band indices $j .{ }^{47}$ The constant-volume (Helmholtz) free energy $F$ can be derived from the partition function via the bridge relation: ${ }^{47}$

$$
F=-k_{B} T \ln Z(T)=U_{L}+U_{V}(T)-T S_{V}(T)
$$

where $U_{V}$ is the vibrational internal energy and $S_{V}$ is the vibrational entropy. The terms $U_{V}(T)$ and $S_{V}(T)$ are temperature-dependent via the partition function, whereas $U_{L}$ is assumed to be temperature independent and is given by the (athermal) total energy from e.g. a density-functional theory (DFT) calculation.

\section{b. The Quasi-Harmonic Approximation}

The harmonic approximation is relatively accurate at low-to-moderate temperatures but is not sufficient to describe quantities that are volume dependent such as the Gibbs free energy and thermal expansion coefficients. The quasi-harmonic approximation (QHA) takes into account the volume dependence of the phonon frequencies by applying the harmonic approximation at a series of compressions and expansions. ${ }^{49,50}$

The Gibbs free energy $G(T, p)^{38}$ is generally a more experimentally-relevant free energy and is calculated as the minimum of $F(T ; V)+p V$ over a range of volumes:

$$
G(T, p)=\min _{V}[F(T ; V)+p V]
$$

In practice, this is achieved by fitting the Helmholtz free energy versus volume $F(T ; V)$ at each temperature $T$ to an expression such as the Birch-Murnaghan equation of state (EoS), which also 
yields the equilibrium free energy $F_{0}$, equilibrium volume $V_{0}$, the bulk modulus $B_{0}$ and the derivative of the bulk modulus with respect to pressure $B_{0}^{\prime}$ at the target temperature: ${ }^{51}$

$$
F(T ; V)=F_{0}(T)+\frac{9 V_{0} B_{0}}{16}\left\{\left[\left(\frac{V_{0}}{V}\right)^{\frac{2}{3}}-1\right]^{3} B_{0}^{\prime}+\left[\left(\frac{V_{0}}{V}\right)^{\frac{2}{3}}-1\right]^{2}\left[6-4\left(\frac{V_{0}}{V}\right)^{\frac{2}{3}}\right]\right\}
$$

Once these quantities are known, other thermal properties can then be derived. ${ }^{47}$

\section{COMPUTATIONAL MODELLING}

First-principles calculations were performed using pseudopotential plane-wave density functional theory (DFT) as implemented in the Vienna $A b$ initio Simulation Package (VASP) code. ${ }^{52}$ Electron exchange and correlation were modelled using the PBEsol functional ${ }^{53}$ with the DFT-D3 dispersion correction, ${ }^{54}$ which previous studies have shown to yield accurate results for the structure and vibrational properties of the tin sulfides. ${ }^{20}$ Initial models of the Pnma ${ }^{55}$ and rocksalt ${ }^{56}$ phases of SnS were taken from Materials Project database, ${ }^{57}$ while the structures of the $\mathrm{Cmcm}$ and $\pi$-cubic phases were obtained from a previous study. ${ }^{20}$ The corresponding phases of SnSe were built from these models by replacing the chalcogen atoms. The models were fully optimized to a tolerance of $10^{-2} \mathrm{eV} \AA^{-1}$ on the forces, with a plane-wave cut-off of $600 \mathrm{eV}$ and the $\boldsymbol{k}$-point sampling summarized in Table 1. An energy convergence criterion of $10^{-8} \mathrm{eV}$ was applied during the electronic self-consistency cycles. The core electrons were modelled using the projector augmented-wave (PAW) pseudopotential method, with the Sn 5s, 5p, and $4 d$ and the $S 3 s / 3 p$ and Se $4 \mathrm{~s} / 4 \mathrm{p}$ electrons included in the valence shell..$^{58}$

Electronic-structure calculations were performed using PBEsol + D3 to investigate the chemical bonding from the composition of the valence band. Self-consistent calculations of the charge density was first performed using the same $\boldsymbol{k}$-point meshes as used for the optimization. To 
obtain the total and atom- and orbital-projected electronic density of states (DoS), eigenvalues were computed non-self-consistently on denser $\boldsymbol{k}$-point mesh with $16 \times$ the number of points, and the tetrahedron method with Blöchl corrections was used for the Brillouin zone integration. To obtain electronic band structures, Brillouin zone paths were generated using the VASPKIT code, ${ }^{59}$ and non-self-consistent calculations performed to evaluate the band energies along strings of $\boldsymbol{k}$ points on the chosen path. The Sumo software was used to visualize and analyze the data. ${ }^{60}$

Lattice-dynamics calculations were carried out on the optimized structures using the Phonopy ${ }^{47}$ software package, which was used to set up and post process supercell finitedisplacement phonon calculations using the supercell expansions and $\boldsymbol{k}$-point sampling in Table 1. ${ }^{61}$ The forces in the displaced structures were calculated using VASP, and an additional chargedensity grid containing $8 \times$ the number of points as the standard grid was used to evaluate the augmentation charges to minimize numerical noise. The force constants of the Pnma and $\pi$-cubic phases were calculated using expansions of the 8- and 64-atom primitive cells, while those of the $\mathrm{Cmcm}$ and rocksalt phases were calculated using expansions of the 8-atom conventional cells and transformation matrices applied to map to the primitive cells during post-processing (Table 2). For the $\pi$-cubic phases of $\mathrm{SnS}$ and $\mathrm{SnSe}$, the $\boldsymbol{k}$-point sampling was reduced to the $\Gamma$ point for the 2 $\times 2 \times 2$ supercell calculations due to the large cells (512 atoms) and consequent computational demand. Tests comparing phonon spectra computed with force constants calculated in the single cells showed that the equivalent reduced $\boldsymbol{k}$-point mesh had a minimal effect on the phonon DoS and calculated free energy (see Figure S2, Supporting Information). 
Table 1. Summary of the technical parameters used for the geometry optimisations and phonon calculations on the four phases of SnS and SnSe: phonon supercell expansion, $\boldsymbol{k}$-point sampling (MP - Monkhorst-Pack $\boldsymbol{k}$-point grid; $\Gamma$-MP $=\Gamma$-centered MP $\boldsymbol{k}$-point grid $^{62}$ ), and plane-wave cutoff.

\begin{tabular}{|c|c|c|c|c|}
\hline \multirow[b]{2}{*}{ Phase } & \multirow{2}{*}{$\begin{array}{l}\text { Phonon SC } \\
\text { (no. atoms) }\end{array}$} & \multicolumn{2}{|c|}{$\boldsymbol{k}$-point sampling } & \multirow[b]{2}{*}{ Cutoff $[\mathrm{eV}]$} \\
\hline & & Optimization & Phonon & \\
\hline Rocksalt & $3 \times 3 \times 3(216)$ & $6 \times 6 \times 6 \Gamma-\mathrm{MP}$ & $2 \times 2 \times 2 \Gamma-\mathrm{MP}$ & 600 \\
\hline Pnma & $1 \times 6 \times 6(288)$ & $4 \times 8 \times 8 \Gamma-\mathrm{MP}$ & $4 \times 2 \times 2 \Gamma-\mathrm{MP}$ & 600 \\
\hline $\mathrm{Cmcm}$ & $6 \times 1 \times 6(288)$ & $8 \times 4 \times 8 \mathrm{MP}$ & $2 \times 4 \times 2 \mathrm{MP}$ & 600 \\
\hline$\pi$-cubic & $2 \times 2 \times 2(512)$ & $4 \times 4 \times 4 \Gamma-\mathrm{MP}$ & $1 \times 1 \times 1 \Gamma-\mathrm{MP}$ & 600 \\
\hline
\end{tabular}

Table 2. Transformation matrices and uniform $\Gamma$-centered $\boldsymbol{q}$-point meshes used to evaluate the phonon frequencies of $\mathrm{SnS}$ and SnSe during post-processing.

\begin{tabular}{|c|c|c|}
\hline Phase & Transformation matrix to primitive cell & $q$-point sampling \\
\hline Rocksalt & $\left(\begin{array}{ccc}0 & 1 / 2 & 1 / 2 \\
1 / 2 & 0 & 1 / 2 \\
1 / 2 & 1 / 2 & 0\end{array}\right)$ & $32 \times 32 \times 32$ Г-МР \\
\hline Pnma & - & $8 \times 48 \times 48 \Gamma-\mathrm{MP}$ \\
\hline $\mathrm{Cmcm}$ & $\left(\begin{array}{ccc}1 / 2 & -1 / 2 & 0 \\
1 / 2 & 1 / 2 & 0 \\
0 & 0 & 1\end{array}\right)$ & $48 \times 8 \times 48 \Gamma-\mathrm{MP}$ \\
\hline$\pi$-cubic & - & $32 \times 32 \times 32 \Gamma-\mathrm{MP}$ \\
\hline
\end{tabular}

Phonon DoS curves were obtained by interpolation of the phonon frequencies onto the $\boldsymbol{q}$ point meshes listed in Table 2, using the linear tetrahedron method for Brillouin-zone integration. Phonon dispersion curves were obtained by interpolation of the phonon frequencies along lines of $\boldsymbol{q}$-points passing between the high symmetry points in the Brillouin zones of the respective primitive cells. Thermodynamic properties were calculated using the phonon frequencies on the same $\boldsymbol{q}$-point grids as used to obtain the DoS curves. Quasi-harmonic calculations were carried out 
by performing phonon calculations on optimized structures with up to $27 \%$ compressions and 15 $\%$ expansions of the cell volume relative to the equilibrium structures. During post processing, the free energy and properties were computed over temperature and pressure ranges of $0-1000 \mathrm{~K}$ and 0-15 GPa respectively.

The ModeMap code ${ }^{63}$ was used to map out the PES along the imaginary harmonic modes in the $\mathrm{Cmcm}$ phases of $\mathrm{SnS}$ and SnSe. 1D and 2D maps for the two principal imaginary modes at the $\mathrm{Y}$ and $\Gamma$-points of the $\mathrm{Cmcm}$ phase were obtained in the conventional cell using a normal-mode coordinate $(Q)$ range of -7.5 to $7.5 \mathrm{amu}^{1 / 2} \AA$ in steps of $0.5 \mathrm{amu}^{1 / 2} \AA$. The $1 \mathrm{D}$ mode maps were used to compute renormalized harmonic frequencies for the imaginary modes using the approach in Ref. ${ }^{63}$. The anharmonic energy levels were computed by solving a 1D Schrödinger equation for an order-20 polynomial fit of the potential, over the $\pm 7.5 \mathrm{amu}^{1 / 2} \AA$ mapped, with 512 eigenstates, using the Fourier method described in Ref. ${ }^{64}$.

The rocksalt and $\pi$-cubic phases are not related by imaginary harmonic modes. A transition path was instead estimated by linearly interpolating the atomic positions in a $2 \times 2 \times 2$ supercell of the rocksalt conventional cell and the distorted $P 2_{1} 3$ structure and using the climbing-image nudged elastic-band (CI-NEB) $)^{65}$ method, starting with six images between the endpoints, to locate the minimum-energy path between them. Each image was optimized until the maximum force on all images was less than $5 \times 10^{-3} \mathrm{eV} \AA^{-1}$. 


\section{RESULTS AND DISCUSSION}

\section{a. Optimized Structures, Lattice Parameters and Lattice Energies}

Representative images of the structures of the four monochalcogenide phases are shown in Figure 1, and the optimized lattice constants and relative energies of the four phases of SnS and SnSe are collected in Tables 3 and 4. In general, our results on SnS are in good agreement with previous DFT calculations using the PBEsol + D3 functional, ${ }^{20}$ and our results on the Pnma, Cmcm and rocksalt phases of $\mathrm{SnSe}$ also agree reasonably well with other theoretical studies using PBE. ${ }^{40,66}$

Our optimized lattice parameters (Table 3) are generally within $0.25-4.99 \%$ of the experimental lattice parameters reported for $\mathrm{SnS}$ and $\mathrm{SnSe}{ }^{30,67}$ The calculated parameters for Pnma SnSe are in relatively good agreement with the DFT studies by Yang et al. ${ }^{44}$ and Pal et al. ${ }^{45}$ although in both studies the calculated lattice constants were overestimated relative to experiment. This can be put down to the tendency of the PBE functional used in these studies to overestimate lattice constants and cell volumes. ${ }^{68}$ Similarly, our calculations are a better match to experiment than PBE calculations on $\pi$-cubic SnSe. ${ }^{17}$

For both chalcogenides, the ground-state structure is the orthorhombic Pnma phase (Table 4, Figure 1a). In this structure, the $\mathrm{Sn}(\mathrm{II})$ atoms adopt a distorted tetrahedral configuration with one site occupied by the active Sn 5s lone pair, leading to a layered structure in which 2D sheets are weakly held together by van der Waals-like interactions. ${ }^{69}$ The adjacent layers in the unit cell stack perpendicular to the long axis, and the crystal is anisotropic due to the different chemical bonding along the three principal axes. ${ }^{1}$

At high temperatures of $878 \mathrm{~K}$ and $807 \mathrm{~K}$, respectively, $\mathrm{SnS}$ and $\mathrm{SnSe}$ both transform reversibly to the orthorhombic $\mathrm{Cmcm}$ structure (Figure 1b),$^{70}$ with a change in formal 
coordination from threefold to fivefold. ${ }^{69}$ The $\mathrm{Cmcm}$ phase also consists of distorted $\mathrm{Sn}$ (II) tetrahedra and 2D layers stacked along one crystal axis, ${ }^{1}$ and can be thought of as a more symmetric form of the Pnma structure. ${ }^{20}$ The lattice energies in Table 4 indicate that $\mathrm{Cmcm}$ SnS and SnSe are 1.73 and $1.39 \mathrm{~kJ} \mathrm{~mol}^{-1}$ per F.U. higher in energy than the Pnma phase. The optimized lattice constants are within 2.14-4.99\% of experimental measurements (Table 3), and we attribute the slightly larger deviation compared to the Pnma phase to thermal expansion at high temperature.

The third proposed phase of $\mathrm{SnS}$ and $\mathrm{SnSe}$ is the $F m \overline{3} m$ rocksalt phase (Figure 1c). Rocksalt $\mathrm{SnS}$ and SnSe were first reported by Mariano et al. in 1967 and were obtained by epitaxial growth on $\mathrm{NaCl}$ substrates. ${ }^{22}$ The observed lattice constants were 6.00 and $5.99 \AA$ for $\mathrm{SnS}$ and SnSe respectively, ${ }^{22}$ to which our optimized lattice constants of 5.71 and $5.91 \AA$ are a reasonable match (Table 3). We note however that we would not necessarily expect a bulk lattice constant to match an epitaxially-grown sample. Our calculated lattice energies suggest rocksalt $\mathrm{SnS}$ is unlikely to be stable in bulk under ambient conditions, at $10.65 \mathrm{~kJ} \mathrm{~mol}^{-1}$ per F.U. above the Pnma phase, whereas rocksalt SnSe is much closer to the convex hull at just $2.52 \mathrm{~kJ} \mathrm{~mol}^{-1}$ per F.U. above the Pnma phase (Table 4). This is in line with previous studies. ${ }^{20,23}$ Rocksalt SnS has also been shown to possess imaginary modes in the phonon dispersion, indicating it to be dynamically unstable in bulk, although these harden and become real under pressure. ${ }^{20}$

Both $\mathrm{SnS}$ and $\mathrm{SnSe}$ were recently reported in the $P 2_{1} 3$ ( $\pi$-cubic) phase (Figure 1d). ${ }^{17-19}$ This phase is accessible through several synthetic routes including aerosol-assisted chemical-vapor deposition, chemical-bath deposition and successive ionic-layer adsorption and reaction. ${ }^{16,19,71}$ Our optimized lattice constants are within $2 \%$ of the measured values of $a=11.7$ and $11.97 \AA$ (Table 3). ${ }^{17,18}$ Unlike the unit cells of the other three polymorphs, which contain up to 8 atoms, the $\pi$-cubic structure has a much larger 64 -atom primitive cell. ${ }^{18}$ The structure is based on a 
distorted rocksalt supercell with a similar local coordination to the Pnma and $\mathrm{Cmcm}$ phases but a $3 \mathrm{D}$ bonding network. ${ }^{20}$ Numerous experimental and theoretical studies have established that these are metastable phases with formation energies close to those of the ground-state Pnma phases. ${ }^{20}$ Our calculations confirm this, placing the lattice energies of $\pi$-cubic $\mathrm{SnS}$ and $\mathrm{SnSe}$ at 2.65 and $2.83 \mathrm{~kJ} \mathrm{~mol}^{-1}$ per F.U. above the corresponding Pnma phases.

Table 3. Optimized lattice parameters of the four phases of SnS (top) and SnSe (bottom) obtained using the PBEsol + D3 functional, with experimental data for comparison; the fourth column shows the $\%$ difference between the optimized and experimental values. ${ }^{\text {a }}$

\begin{tabular}{|c|c|c|c|}
\hline \multicolumn{4}{|c|}{$\mathrm{SnS}$} \\
\hline \multirow[t]{2}{*}{ Phase } & $\begin{array}{c}\text { PBEsol + D3 } \\
\text { (this work) }\end{array}$ & Experiment & \multirow[t]{2}{*}{$\%$ Difference } \\
\hline & $a, b, c(\AA)$ & $a, b, c(\AA)$ & \\
\hline Rocksalt & 5.71 & $5.80^{22}$ & -1.55 \\
\hline Pnma & $11.00,3.97,4.20$ & $11.18,3.98,4.33^{67}$ & $-1.70,-0.25,-3.00$ \\
\hline $\mathrm{Cmcm}$ & $4.02,11.18,4.01$ & $4.18,11.48,4.15^{72}$ & $-3.83,-2.61,-3.37$ \\
\hline$\pi$-cubic & 11.40 & $11.60^{18}$ & -1.72 \\
\hline \multicolumn{4}{|c|}{$\mathrm{SnSe}$} \\
\hline \multirow[t]{2}{*}{ Phase } & $\begin{array}{l}\text { PBEsol + D3 } \\
\text { (this work) }\end{array}$ & Experiment & \multirow[t]{2}{*}{$\%$ Difference } \\
\hline & $a, b, c(\AA)$ & $a, b, c(\AA)$ & \\
\hline Rocksalt & 5.91 & $5.99^{22}$ & -1.34 \\
\hline Pnma & $11.35,4.12,4.34$ & $11.57,4.19,4.46^{30}$ & $-1.90,-1.67,-2.69$ \\
\hline $\mathrm{Cmcm}$ & $4.19,11.45,4.17$ & $4.41,11.71,4.32^{72}$ & $-4.99,-2.22,-3.47$ \\
\hline$\pi$-cubic & 11.78 & $11.97^{17}$ & -1.59 \\
\hline
\end{tabular}

${ }^{a}$ Lattice constants in Ref. ${ }^{72}$ are reported at $905 \mathrm{~K}$ and $825 \mathrm{~K}$ and are therefore expected to include significant thermal expansion. 
Table 4. Lattice energy differences $\Delta U_{L}$ of the four phases of $\mathrm{SnS}$ and SnSe relative to the groundstate Pnma structure calculated using PBEsol + D3.

\begin{tabular}{lcc}
\hline \multirow{2}{*}{ Phase } & \multicolumn{2}{c}{$\Delta U_{L}\left[\mathrm{~kJ} \mathrm{~mol}^{-1}\right.$ per F.U.] } \\
\cline { 2 - 3 } & $\mathrm{SnS}$ & SnSe \\
\hline Pnma & 0.00 & 0.00 \\
Cmcm & 1.73 & 1.39 \\
\hline Rocksalt & 10.65 & 2.42 \\
\hline$\pi$-cubic & 2.65 & 2.83 \\
\hline
\end{tabular}

Based on the lattice energy differences per formula unit, $\Delta U_{L}$, listed in Table 4, we find the following energetic stability orderings: SnS - Pnma $<$ Cmcm $<\pi$-cubic $<$ rocksalt; SnSe - Pnma $<\mathrm{Cmcm}<$ rocksalt $<\pi$-cubic. This is expected given that the Pnma phase is the naturally-occurring form of both compounds, the $\mathrm{Cmcm}$ phase is obtained at elevated temperature, and the $\pi$-cubic phase is a known metastable structure. ${ }^{20}$ There is however a notable difference in the stabilities of the rocksalt phases. This structure is the least energetically-stable phase of SnS by a considerable margin, whereas rocksalt $\mathrm{SnSe}$ is much closer to the convex hull and more energetically stable than the $\pi$-cubic structure, suggesting it may compete with it as a metastable phase.

The comparatively rich monochalcogenide phase spaces result from the stereochemical activity of the Sn $5 \mathrm{~s}$ lone pair. Sn has the valence electronic configuration $4 \mathrm{~d}^{10} 5 \mathrm{~s}^{2} 5 \mathrm{p}^{2}$, while the chalcogens have the configuration $n \mathrm{~s}^{2} n \mathrm{p}^{4}$ with $n=3$ and 4 for S and Se, respectively. When these elements form covalent bonds, Sn loses two electrons to S/Se resulting in Sn and chalcogen configurations of $4 \mathrm{~d}^{10} 5 \mathrm{~s}^{2} 5 \mathrm{p}^{0}$ and $n \mathrm{~s}^{2} n \mathrm{p}^{6}$. The $\mathrm{Sn}(\mathrm{II})$ cation in both $\mathrm{SnS}$ and SnSe therefore has a chemically-inactive lone pair of electrons in the 5s orbital. A coupling between the $\mathrm{Sn} 5 \mathrm{~s}$ and $\mathrm{Sn}$ $5 \mathrm{p}$ orbitals, mediated through the antibonding interaction between the Sn $5 \mathrm{~s}$ and anion $\mathrm{p}$ orbitals, produces an asymmetric electron density on Sn and drives structural distortions away from a centrosymmetric environment. The energy gap between $\mathrm{Sn} 5 \mathrm{~s}$ and $\mathrm{Se} 4 \mathrm{p}$ is larger than that between 
the $\mathrm{Sn} 5 \mathrm{~s}$ and S 3p, which reduces the stability of the distorted structures relative to the symmetric structures with higher coordination numbers. ${ }^{9}$ This difference results in the centrosymmetric rocksalt SnS being strongly destabilized compared to the other distorted phases, whereas the energetic preference for the distorted structures is much smaller in SnSe.

\section{b. Electronic Structure}

In order to obtain more physical insight into the relative structural stability of the monochalcogenides, we performed electronic-structure calculations to study the bonding character from the compositions of the valence bands. Figure 2 shows calculated electronic band structures and density of states (DoS) curves for the eight systems.

The electronic structure of the tin chalcogenides has been analyzed in detail elsewhere. ${ }^{2,9,17,21,40,44,66,73-80}$ The electronic DoS and band structures of the four monochalcogenide phases possess some common features. The lower parts of the valence bands from around -8 to -4 $\mathrm{eV}$ comprise mainly $\mathrm{Sn} 5 \mathrm{~s}$ and $\mathrm{S} / \mathrm{Se} 3 \mathrm{p} / 4 \mathrm{p}$ states, whereas the upper parts of the valence bands from $\sim-4$ to $0 \mathrm{eV}$ are composed of $\mathrm{S} 3 \mathrm{p} / \mathrm{Se} 4 \mathrm{p}$ and $\mathrm{Sn} 5 \mathrm{p}$ states with smaller contributions from $\mathrm{Sn}$ 5s states. In all eight system the $\mathrm{S} / \mathrm{Se}$ valence s states and the $\mathrm{Sn} 4 \mathrm{~d}$ states occupy isolated bands well below the valence-band maximum (VBM). This indicates that the valence bonding is predominantly through the Sn and S/Se p states, and the projected DoS curves and band structures indicate a larger contribution from the chalcogenide anions. In keeping with this, the conduction band minima $(\mathrm{CBM})$ in the systems are composed of $\mathrm{Sn}$ and chalcogen $\mathrm{p}$ states, with a larger contribution from the metal states. These observations are in agreement with previous studies. ${ }^{2,9,76-}$ 80,17,21,40,44,66,73-75 The calculations clearly show an involvement of the $\mathrm{Sn} 5$ s states in the valence 
band, as predicted from the revised lone-pair model, ${ }^{9,81}$ and the presence of Sn 5s states at the valence band edge is a hallmark of the stereochemically-active lone pair. ${ }^{76}$

In principle, marked differences in the stability of rocksalt $\mathrm{SnS}$ and $\mathrm{SnSe}$ relative to the distorted phases could be explained by differences in the relative energies of the chalcogenide anion $\mathrm{p}$ states and the $\mathrm{Sn} 5 \mathrm{~s}$ states. Indeed, it can be seen that there is a slightly lower participation from the $\mathrm{Sn} 5 \mathrm{~s}$ states at the VBM in rocksalt $\mathrm{SnSe}$, which indicates a reduced driving force for structural distortions and thus less destabilization of the centrosymmetric structure. (For clarity, enlarged projected DoS plots for rocksalt $\mathrm{SnS}$ and SnSe are provided in Figure S3, Supporting Information.)

Some notable differences can also be observed between the different phases. The band dispersions of the $\mathrm{Cmcm}$ phases clearly show higher degeneracy than those of the Pnma phases. Similarly, the Rocksalt phases show high degeneracy in the dispersions and more sharply defined features in the DoS in comparison to the orthorhombic phases, reflecting its higher symmetry. We note that the bandgaps predicted in these calculations (Table S1, Supporting Information) are lower than the experimental values, and also that several of the structures are incorrectly predicted to be metallic with the VBM and CBM touching at some points in the Brillouin zone. This is due to the well-known failure of (semi-)local DFT functionals to predict accurate bandgaps. ${ }^{82}$ Despite this, the composition of the valence bands are in line with other theoretical studies, ${ }^{2,9,76-}$ $80,17,21,40,44,66,73-75$ including studies combining high-level theory and measurements, ${ }^{83}$ so we consider the present PBEsol + D3 calculations to be sufficient for discussing the chemical bonding. For completeness, the bandgaps obtained from our calculations are tabulated and compared to measurements and other theoretical studies in Table S1, Supporting Information. 

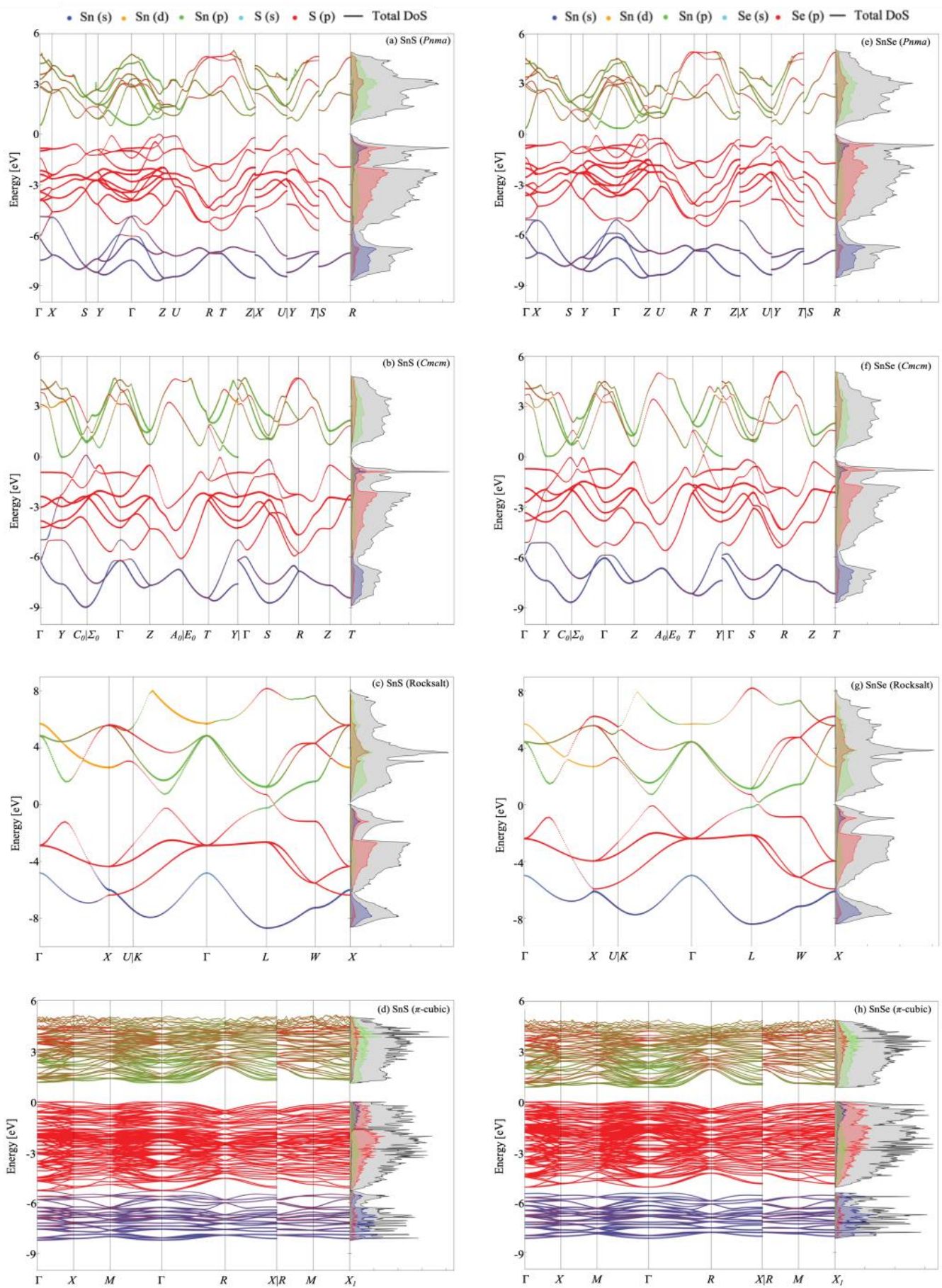

Figure 2. Calculated atom- and orbital-projected electronic band structures and density of states (DoS) curves of the four phases of SnS and SnSe: (a)/(e) Pnma, (b)/(f) Cmcm, (c)/(g) rocksalt and (d)/(h) $\pi$-cubic. The states are colored as follows: $\operatorname{Sn}(s)$ - blue, $\operatorname{Sn}(d)$ - yellow, Sn (p) - green, $\mathrm{S} / \mathrm{Se}(\mathrm{s})$ - cyan, S/Se(p) - red, and total DoS - black. 


\section{c. Harmonic Lattice Dynamics}

\section{i. Dynamical Stability}

Systems with imaginary harmonic modes (corresponding to negative eigenvalues of the dynamical matrix) are dynamically unstable and represent local maxima on the structural potentialenergy surface (PES). The phonon dispersion and DoS curves can therefore be used to assess the dynamical stability of a material.

Figure 3 shows the DoS and dispersion curves of each the four phases of SnS and SnSe. The phonon spectra of the analogous $\mathrm{SnS}$ and SnSe phases are similar, but the heavier chalcogen and weaker bonding interactions in the selenides results in a smaller range of phonon frequencies. Projections of the DoS onto the $\mathrm{Sn}$ and $\mathrm{S} / \mathrm{Se}$ atoms shows that the lower-frequency branches are mostly due to motions of the heavier Sn atoms, while the higher-frequency branches are associated with $\mathrm{S} / \mathrm{Se}$. For some of the systems, there is a clear separation between the low- and high-frequency branches in the dispersion, often referred to as a "phonon bandgap" by analogy to electronic band structures.

For the Pnma and $\pi$-cubic phases of SnS and SnSe there are no imaginary modes in the dispersion and DoS curves, indicating that these structures are dynamically stable under ambient conditions. This is expected given that they correspond to the low-temperature ground-state and known metastable phases, respectively. The phonon dispersions of the high-temperature $\mathrm{Cm} \mathrm{cm}$ phases of $\mathrm{SnS}$ and $\mathrm{SnSe}$ show two principal imaginary modes at the zone center $(\Gamma)$ and the zoneboundary wavevector $Y$. This is also expected and is consistent with previous experimental and theoretical studies. ${ }^{20,63}$ The imaginary modes constitute a very small part of the DoS and are restricted to well-defined phonon wavevectors in the dispersion. The $\mathrm{Cmcm}$ phase is a low-lying hilltop on the structural PES which links equivalent distorted Pnma global minima. At high 
temperatures the system has sufficient thermal energy to transition rapidly between these minima, and the $\mathrm{Cmcm}$ phase is observed crystallographically as an "average" structure. This corresponds to a second-order phase transition. ${ }^{49}$ The main difference between the two chalcogenides is again in the rocksalt phase. Rocksalt SnSe has no imaginary modes and is therefore dynamically stable, whereas $\mathrm{SnS}$ has an imaginary mode at the zone-boundary $X$ wavevector, indicating that it is dynamically unstable under ambient conditions. As for the $\mathrm{Cmcm}$ phase, the proportion of these modes is small in the overall DoS. The contrasting dynamical stability of rocksalt $\mathrm{SnS}$ and $\mathrm{SnSe}$ can be rationalized by the Sn lone pair activity, as discussed in the previous section.

Our conclusions on the dynamical stability of all the phases of $\mathrm{SnS}$ are consistent with our previous studies, ${ }^{20}$ and our findings on the dynamical stability of the orthorhombic phases of SnSe are consistent with the theoretical studies by Lu. et al. ${ }^{73}$ The dynamical stability of the rocksalt and $\pi$-cubic phases of SnSe have also been confirmed in theoretical studies by Wang et al. ${ }^{23}$ and Argaman et al. ${ }^{84}$ respectively.

\section{ii. Thermodynamic Stability: Helmholtz Free Energy}

The harmonic lattice dynamics calculations also allow the thermodynamic stability of the phases to be examined using the temperature-dependent Helmholtz free energy. Figure 4 compares the free energies of the $\mathrm{Cmcm}, \pi$-cubic and rocksalt phases of $\mathrm{SnS}$ and $\mathrm{SnSe}$ as a function of temperature relative to the ground-state Pnma phase. For both chalcogenides, the free energies predict a Pnma $\leftrightarrow \mathrm{Cmcm}$ phase transition at elevated temperature. The predicted transition temperatures are $T_{(P n m a \rightarrow C m c m)}=490$ and $460 \mathrm{~K}$ for $\mathrm{SnS}$ and SnSe respectively, compared to the literature values of 878 and $807 \mathrm{~K} .^{70}$ The calculations therefore correctly predict that the second- 
order phase transition of $\mathrm{SnS}$ occurs at a higher temperature than $\mathrm{SnSe}$, but the calculated temperatures are both lower than the experimental ones.

This difference could be appointed to the harmonic approximation and the neglect of thermal expansion at finite temperature, to small errors in the DFT energies and phonon frequencies, or to the second-order nature of the phase transition. In the latter case, since the $\mathrm{Cm} \mathrm{cm}$ phase is a maximum on the PES, the temperature at which it is detected will depend on the "hopping rate" between the Pnma minima, and thus the size of the energetic barrier, as well as the temporal resolution of the experimental measurements.

The $\pi$-cubic structure remains metastable up to $1000 \mathrm{~K}$. The energy difference with respect to the Pnma phases is approximately constant for $\mathrm{SnS}$, whereas for $\mathrm{SnSe}$ the energy difference decreases significantly at higher temperatures. The overall free-energy difference between the $\pi$ cubic and Pnma structure is lower in SnS, implying that this phase is relatively more stable for $\mathrm{SnS}$ than for SnSe.

A major difference between the two compounds is again seen for the rocksalt structure. The largest energy difference for rocksalt $\mathrm{SnS}$ is $\sim 10 \mathrm{~kJ} \mathrm{~mol}^{-1}$ per F.U. at $0 \mathrm{~K}$, similar to the lattice energy differences, whereas that for SnSe the difference is much smaller and reduced by $\sim 20 \%$ compared to the lattice energies to $2 \mathrm{~kJ} \mathrm{~mol}^{-1}$ per F.U. (i.e. rocksalt $\mathrm{SnSe}$ is stabilized relative to the Pnma phase due to its vibrational free energy). This places the rocksalt phase of SnSe closer to the Pnma phase than the known metastable $\pi$-cubic structure. Interestingly, rocksalt SnSe almost becomes lower in energy than Pnma at $1000 \mathrm{~K}$, although at this temperature the Cmcm phase is lower in energy than both competing phases. Our results for $\mathrm{SnS}$ are consistent with our previous theoretical study, albeit with a small difference in the predicted transition temperature to the $\mathrm{Cm} \mathrm{cm}$ phase. $^{20}$ 

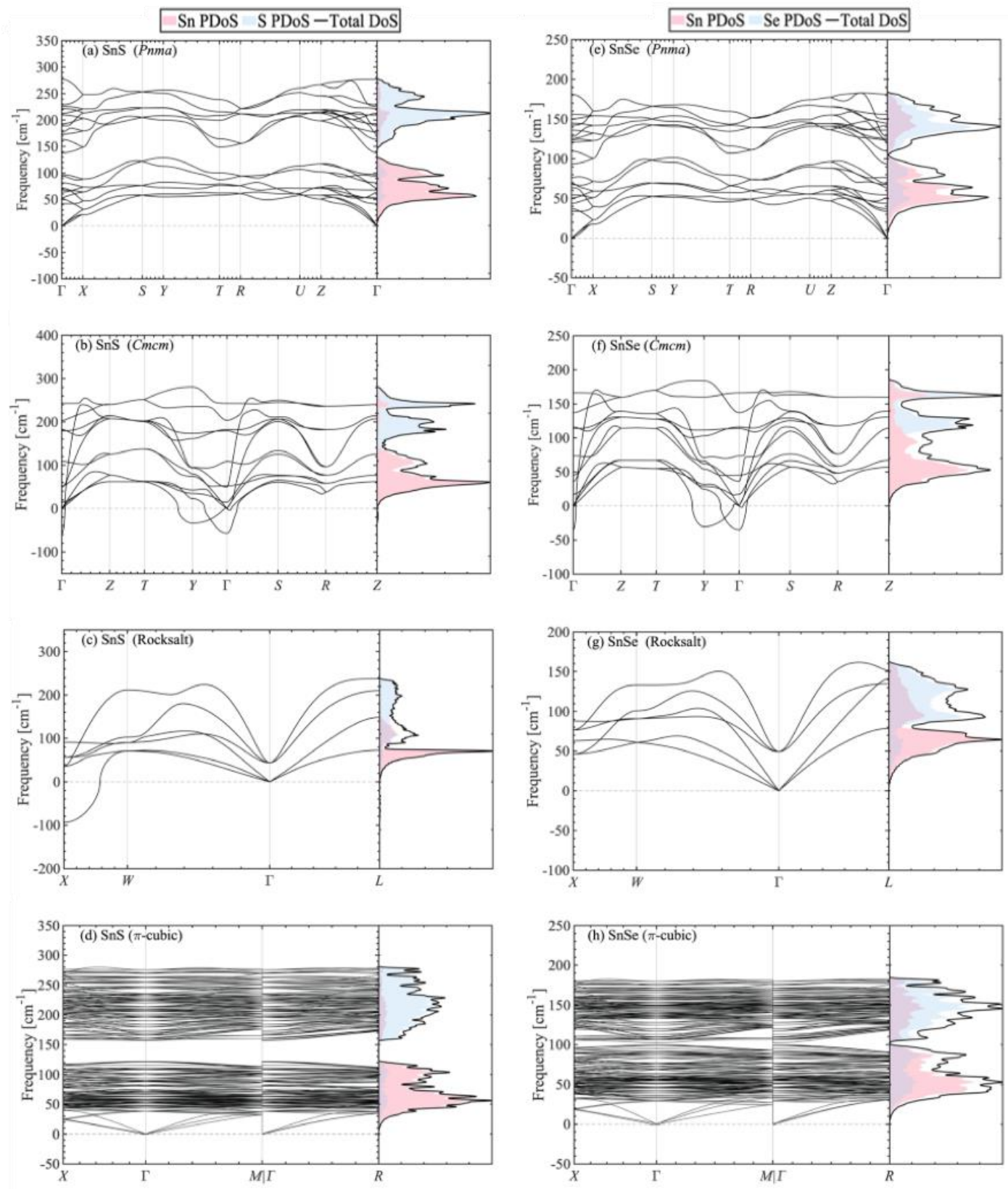

Figure 3. Calculated equilibrium phonon dispersion and density of states (DoS) curves of the four phases of SnS and SnSe: (a)/(e) Pnma, (b)/(f) Cmcm, (c)/(g) Rocksalt and (d)/(h) $\pi$-cubic. The partial density of states (PDoS) projected onto the $\mathrm{Sn}$ and $\mathrm{S} / \mathrm{Se}$ atoms is overlaid on the DoS plots as filled curves with pink and blue shading, respectively. 

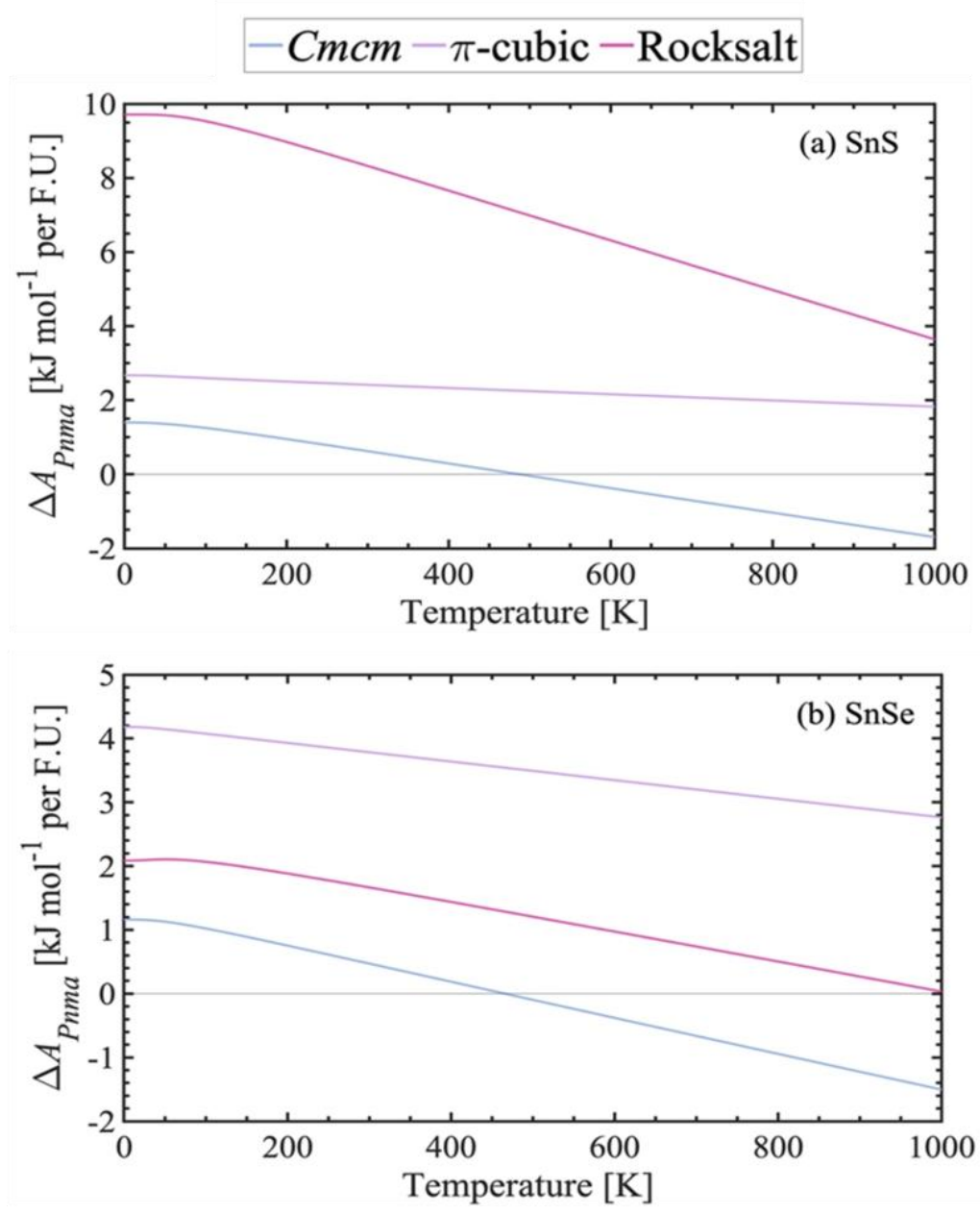

Figure 4. Helmholtz free energy differences $\triangle A$ Pnma between the Cmcm (blue), $\pi$-cubic (purple) and rocksalt (pink) phases of (a) SnS and (b) SnSe relative to the Pnma phase.

\section{iii. Impact of soft modes on the free energies}

The imaginary harmonic modes in the $\mathrm{Cmcm} \mathrm{SnS} / \mathrm{SnSe}$ and rocksalt $\mathrm{SnS}$ dispersions are excluded from the thermodynamic partition function and thus do not contribute to the Helmholtz energy. Since the imaginary modes in $\mathrm{Cmcm} \mathrm{SnS}$ and $\mathrm{SnSe}$ are restricted to well-defined phonon wavevectors and represent only a small fraction of the overall phonon density of states (DoS), we do not expect this to have a significant impact on the calculated thermodynamic properties. However, to quantify this we used the method outlined in Ref. ${ }^{63}$ to perform a basic phonon 
"renormalization" of these modes. In this scheme, the PES along the soft modes is mapped explicitly and the anharmonic energy levels are determined by solving a 1D Schrödinger equation. A temperature-dependent effective harmonic frequency that reproduces the contributions of the modes to the thermodynamic partition function is then obtained and used to correct the harmonic force constants. A similar approach has been shown to predict reasonable transition temperatures for the $P 21 / \mathrm{n} \rightarrow$ Immm phase transition in cryolite, which occurs via a similar mechanism to the Pnma $\leftrightarrow$ Cmcm transition in $\mathrm{SnS}$ and SnSe. ${ }^{85}$
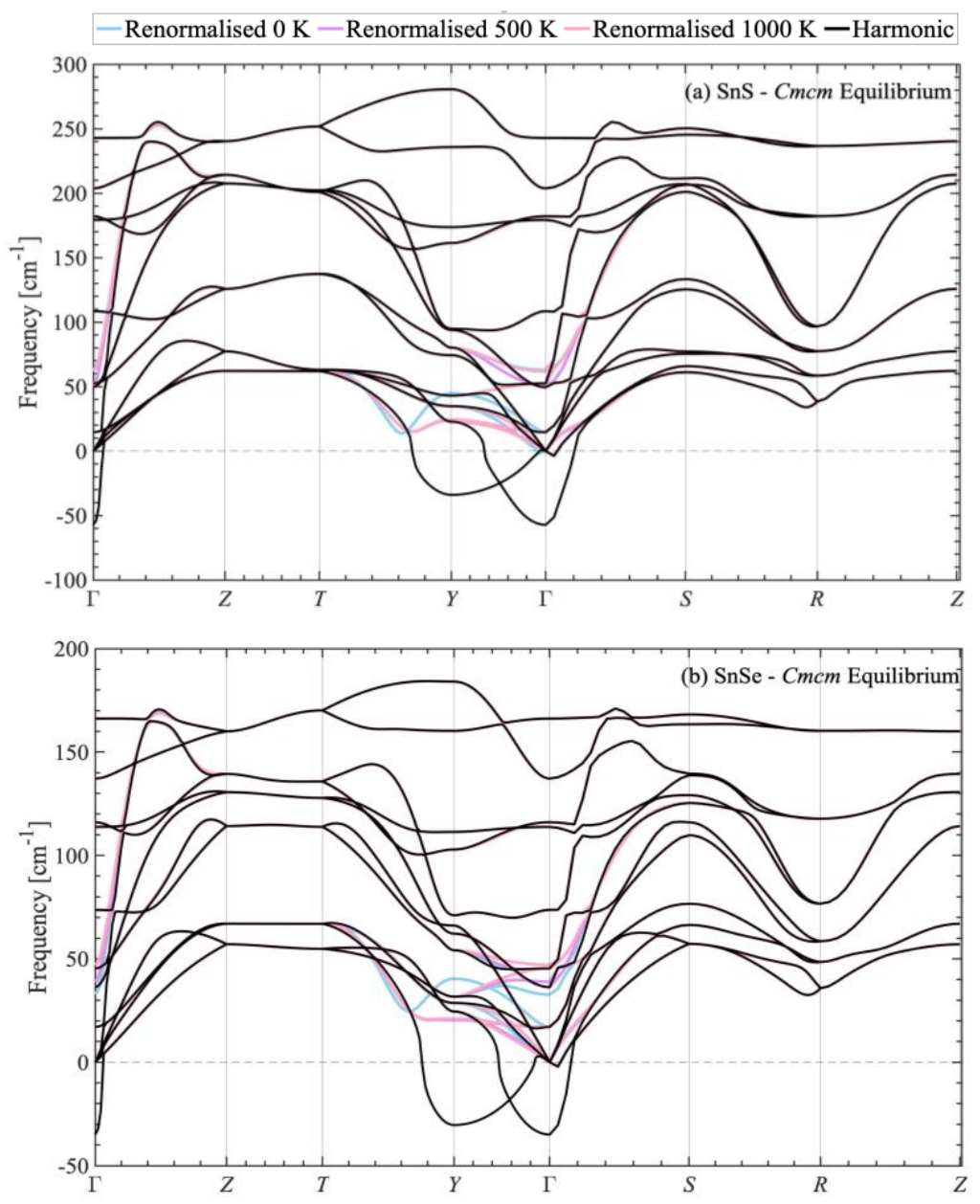

Figure 5. Comparison of the harmonic (black) and renormalized phonon dispersion curves of Cmcm (a) SnS and (b) SnSe (b) at $T=0 \mathrm{~K}$ (blue), $500 \mathrm{~K}$ (purple) and $1000 \mathrm{~K}$ (pink). 
Figure 5 compares the harmonic phonon dispersion curves of $\mathrm{Cmcm} \mathrm{SnS}$ and $\mathrm{SnSe}$ with dispersions computed after renormalizing the soft modes at 0,500 and $1000 \mathrm{~K}$. This demonstrates that the approach effectively shifts the imaginary modes to real frequencies. Figure $\mathbf{6}$ compares the Helmholtz free energy difference $\triangle A_{\text {Pnma }}$ between the Pnma and $\mathrm{Cmcm}$ phases before and after renormalization. For completeness, we also show, in the Supporting Information, the calculated renormalized frequencies of the two modes as a function of temperature (Figure S4) and the anharmonic double-well potentials along the two modes with the eigenvalues obtained from the solver (Figure S5). As can be seen, the energy differences are very similar, and the transition temperatures are changed by maximum of $20 \mathrm{~K}$, which confirms that neglecting the soft modes when computing the harmonic free energies does not significantly affect the result. In light of this finding, and given that rocksalt $\mathrm{SnS}$ is not energetically competitive with the other phases of $\mathrm{SnS}$, we did not investigate the impact of renormalizing the imaginary modes in this phase.

We note that this approach to renormalization is somewhat "crude" in comparison to schemes such as the temperature-dependent effective potential (TDEP) ${ }^{86}$ stochastic self-consistent harmonic approximation (SSCHA) ${ }^{87}$ and self-consistent $a b$ initio lattice dynamics (SCAILD) ${ }^{88}$ techniques. However, these more sophisticated methods are considerably more computationally demanding and calculate temperature-dependent frequencies for all the harmonic modes, which would mean that all the chalcogenide phases would have to be treated using the same approach for the calculated free energies to be comparable. Since the present study focusses on predictions from the QHA, we did not investigate any of these alternatives. 

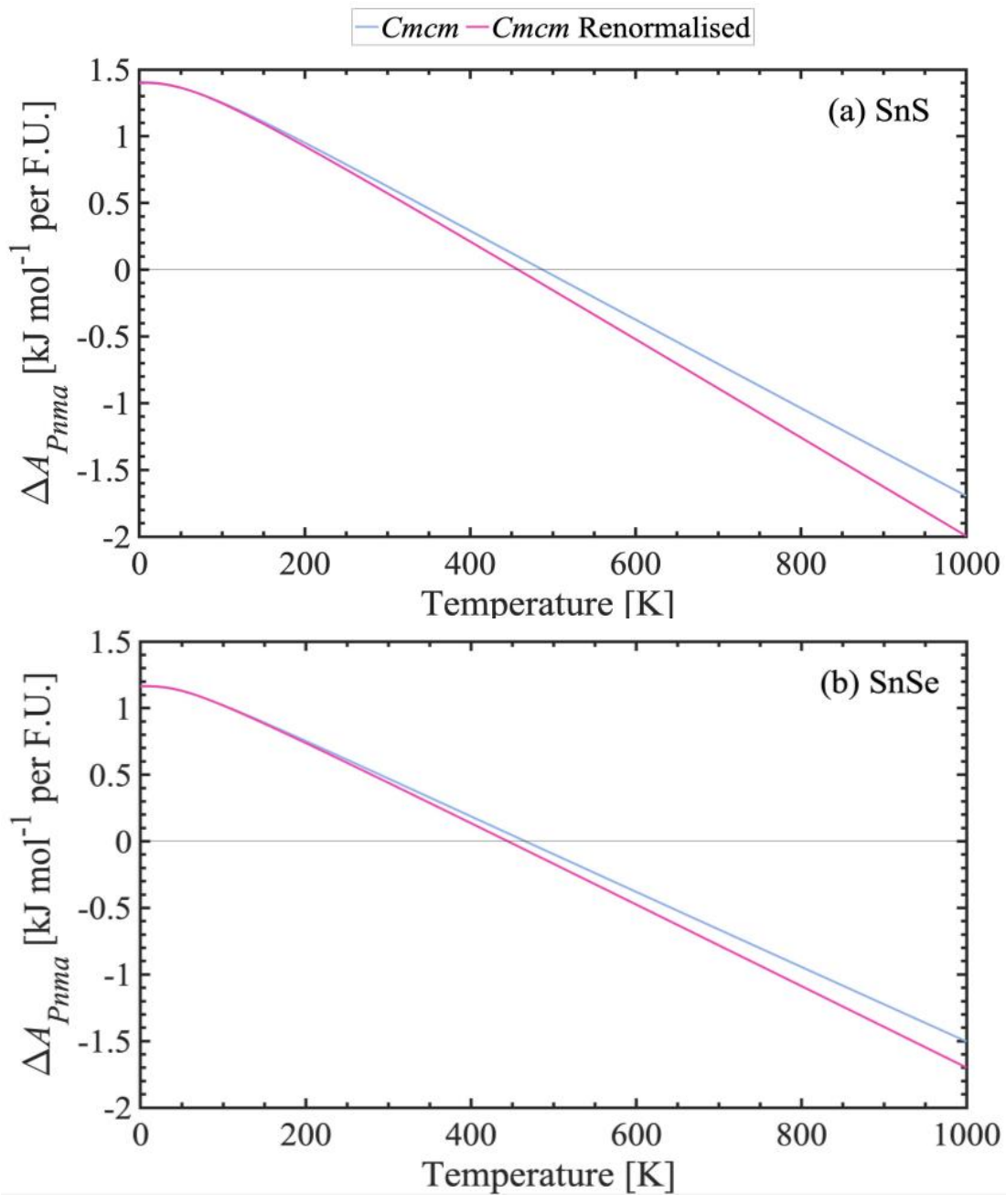

Figure 6. Helmholtz free energy differences $\triangle A$ Pnma between the Pnma and Cmcm phases of (a) $\mathrm{SnS}$ and (b) SnSe before (blue) and after renormalisation (pink).

d. Quasi-Harmonic Lattice Dynamics

To examine the volume dependence of the phonon frequencies, further calculations were performed on the eight compounds with a series of $27 \%$ compressions and $15 \%$ expansions about the optimized equilibrium volumes. 


\section{i. Volume Dependence of the Phonon Dispersion and DoS: Dynamical Stability}

The volume dependence of the phonon DoS and dispersion curves can be used to investigate the effect of pressure on the dynamical stability. Figure 7 compares the phonon spectra of the rocksalt and $\mathrm{Cmcm}$ phases of $\mathrm{SnS}$ as a function of volume, and similar comparisons for all four phases of $\mathrm{SnS}$ and $\mathrm{SnSe}$ are presented in Figure S6 (Supporting Information).
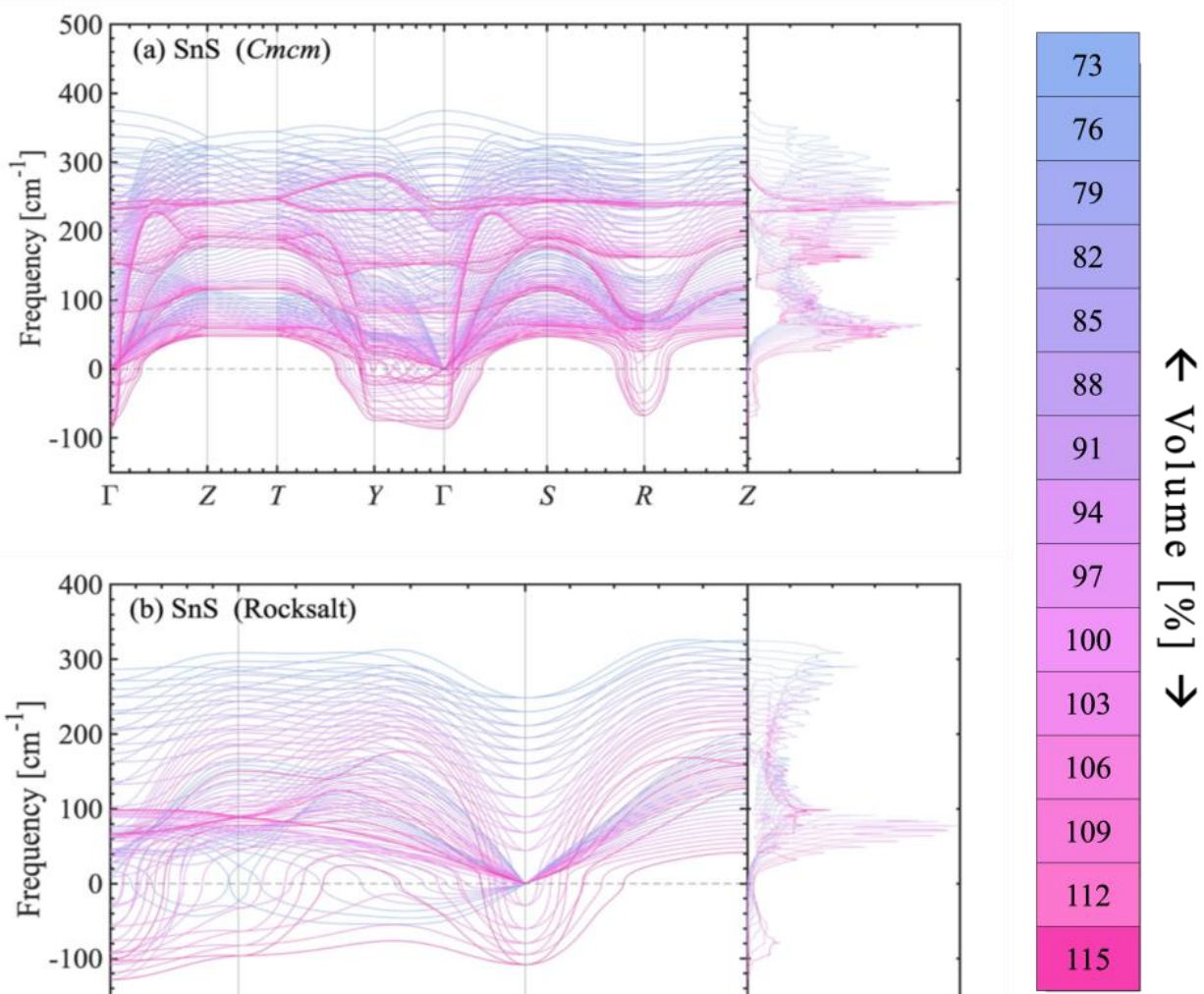

Figure 7. Phonon dispersion and density of states curves of (a) $\mathrm{Cmcm}$ and (b) rocksalt $\mathrm{SnS}$ computed for up to $27 \%$ compression and $15 \%$ expansion of the volume about the calculated equilibrium. Similar analyses for all four phases of $\mathrm{SnS}$ and $\mathrm{SnSe}$ are presented in Figure S6 (Supporting Information). 
The phonon frequencies generally decrease (soften) with increasing volume, as the interatomic forces decrease with increasing bond lengths, and increase (harden) under compression. The $\pi$-cubic phases remain dynamically stable over the full range of calculated volumes. The imaginary modes in the $\mathrm{Cmcm}$ phases become real under compression and disappear under $\sim 12 \%$ compression, indicating that this phase may be dynamically stabilized under moderate pressure, which we calculated to be around $8 \mathrm{GPa}$ for both structures. The imaginary modes in rocksalt $\mathrm{SnS}$ also harden upon compression, as reported in previous theoretical studies, ${ }^{20}$ which indicates that this phase may also be dynamically stabilized under pressure. The present calculations suggest that the imaginary mode at $X$ in the rocksalt phase fully hardens under $\sim 15 \%$ compression, which corresponds to a lattice constant of $5.43 \AA$. A similar effect could be achieved with a reduction in the lattice constant when grown epitaxially and lattice matching to a substrate.

\section{ii. Thermodynamic Stability at Ambient Pressure: Gibbs Energy}

The Gibbs free energies obtained from the QHA were used to assess the thermodynamic stability of the different monochalcogenide phases in the same way as the Helmholtz energies. The Gibbs free energy is at constant pressure, and as such provides a more experimentally relevant means of comparing stabilities.

Figure 8 compares the Gibbs energies of the $\mathrm{Cmcm}, \pi$-cubic and rocksalt phases of $\mathrm{SnS}$ and $\mathrm{SnSe}$ to the ground-state Pnma phase. The calculations again predict a phase-transition from the Pnma to the Cmcm phase, but this time at lower transition temperatures of $T_{(P n m a \rightarrow C m c m)}=440$ and $326 \mathrm{~K}$ for $\mathrm{SnS}$ and $\mathrm{SnSe}$ respectively. Both are lower than those predicted using the Helmholtz free energies and therefore deviate further from the experimental measurements. This suggests that 
the misprediction is likely due to the nature of the phase transition rather than to the approximation of using the Helmholtz energies of the equilibrium structures.

We note in passing that the QHA calculations predict an expansion in the volume of the $\mathrm{Cmcm}$ phases at higher temperature, resulting in better agreement with the experimental measurements. The predicted lattice constants are $a=4.02, b=11.42$ and $c=4.05 \AA$ for $C m c m$ SnS at $905 \mathrm{~K}$, and $a=4.23 b=11.70$ and $c=4.21 \AA$ for $C m c m$ SnSe at $825 \mathrm{~K}$, which decrease the error with respect to the measurements from $3.27 \%$ to $1.99 \%$ for $\mathrm{SnS}$ and from $2.83 \%$ to $1.44 \%$ for SnSe.

For both chalcogenides the Gibbs energies also predict that the $\pi$-cubic phases remain metastable over the $0-1000 \mathrm{~K}$ temperature range considered in the calculations. As for the Helmholtz free energies, the energy difference with respect to the Pnma structure is approximately constant for $\mathrm{SnS}$ but decreases at higher temperatures for SnSe. The overall Gibbs energy difference between the $\pi$-cubic and Pnma structures is lower for SnS, again indicating that the $\pi$ cubic phase of $\mathrm{SnS}$ is more stable than that of $\mathrm{SnSe}$.

On the other hand, we observe a notable difference in the Gibbs energies of the rocksalt phases compared to the Helmholtz energies. While the maximum value of $\Delta G_{P n m a}$ for rocksalt SnSe is around $2 \mathrm{~kJ} \mathrm{~mol}^{-1}$ per F.U. at $0 \mathrm{~K}$, a steeper decrease in the Gibbs energy with temperature leads to this phase becoming lower in energy than the Pnma phase at $440 \mathrm{~K}$. This is consistent with other theoretical studies, which showed that in SnSe the energy of the rocksalt phase only slightly deviates from that of the ground-state Pnma phase. ${ }^{62}$ This suggests that quasi-harmonic effects (i.e. thermal expansion) may lead to rocksalt SnSe being energetically competitive with the other phases at high temperature. Although the $\mathrm{Cmcm}$ phase has a lower free energy than the rocksalt phase, the $\mathrm{Cmcm}$ structure is dynamically unstable at ambient pressure; if the rocksalt 
phase corresponds to the stable phase with the lowest free energy at elevated temperature, and is accessible from the $\mathrm{Cmcm}$ phase on the structural PES, we might expect this phase to be observed at high temperature in SnSe. In contrast, rocksalt $\mathrm{SnS}$ remains higher in energy than the Pnma phase across the full range of temperatures examined.
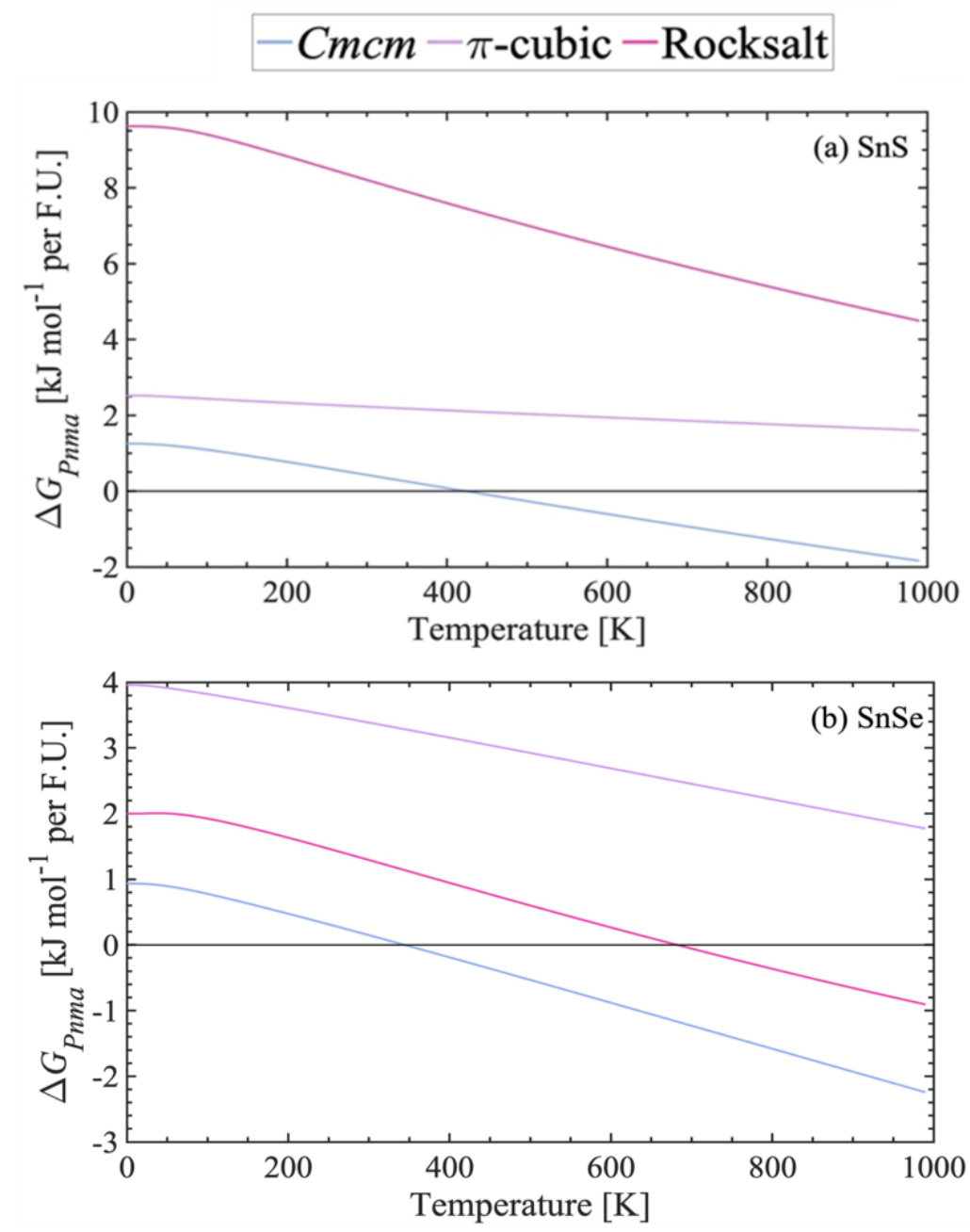

Figure 8. Gibbs energy difference $\Delta G_{P n m a}$ between the $C m c m$ (blue), $\pi$-cubic (purple) and rocksalt (pink) phases of (a) SnS and (b) SnSe relative to the Pnma phase.

We also calculated the Gibbs energy of the $\mathrm{Cm} \mathrm{cm}$ phases relative to the Pnma phases using the renormalization method used to correct the Helmholtz energies of the equilibrium structures 
(Supporting Information, Figure S7), and again find that the transition temperatures are only very slightly altered by this correction.

\section{iii. Temperature/Pressure Phase Diagrams}

By adding a $p V$ term to the quasi-harmonic Gibbs free energies, we can also use the QHA to study the effect of pressure on the relative stabilities of the materials and to compute, from first principles, 2D temperature-pressure phase diagrams. Phase diagrams for $\mathrm{SnS}$ and $\mathrm{SnSe}$, covering temperatures from 0-1000 $\mathrm{K}$ and pressures from 0-15 GPa, are presented in Figure 9. Based on the Gibbs energies, only the orthorhombic Pnma (pink) and Cmcm (blue) structures appear on these diagrams. For both $\mathrm{SnS}$ and $\mathrm{SnSe}$, the Pnma $\leftrightarrow \mathrm{Cmcm}$ transition temperature is predicted to decrease with increasing pressure, and the transition temperature is consistently lower for SnSe than for SnS. Beyond a certain pressure, which appears to be around $11 \mathrm{GPa}$ for $\mathrm{SnS}$ and $7 \mathrm{GPa}$ for SnSe, the $\mathrm{Cmcm}$ structure becomes lower in energy at all temperatures. We note that we checked the structures of both the Pnma and $\mathrm{Cmcm}$ phases at compressed volumes to confirm that there were no spontaneous transformations between them - optimised equilibrium structures of Pnma and $\mathrm{Cmcm}$ corresponding to pressures of approximately 0, 5, 10 and $15 \mathrm{GPa}$ are shown in Figure S8 in the Supporting Information.

We also show on the phase diagrams the regions where the $\mathrm{Cmcm}$ phase is predicted to be dynamically stable, which identify that this phase is both dynamically and thermodynamically stable above $11 \mathrm{GPa}$ for $\mathrm{SnS}$ and $9 \mathrm{GPa}$ for SnSe. We also find a small region of the SnSe phase diagram where the rocksalt structure is more thermodynamically stable than the Pnma phase and the $\mathrm{Cmcm}$ remains dynamically unstable. This region spans a temperature range of $\sim 700$ to 1000 
$\mathrm{K}$ and pressures up to $2 \mathrm{GPa}$. We note that the regions of the phase diagram where the $\mathrm{Cmcm}$ phase is energetically stable but dynamically unstable are those where we would expect crystallography to show an average $\mathrm{Cmcm}$ structure, but spectroscopic techniques sensitive to local structure, such as X-ray absorption spectroscopy and pair-distribution function measurements, to indicate a different local structure. The region of the SnSe phase diagram where the calculations predict the rocksalt phase to be lower in energy than the Pnma phase would therefore be particularly interesting to study.

Several studies have attempted to estimate the transition pressure from the Pnma to the $\mathrm{Cmcm}$ phase. By calculating relative enthalpies of formation, Yang et al. predicted a pressureinduced Pnma $\leftrightarrow \mathrm{Cmcm}$ phase transition in SnSe at around $22 \mathrm{GPa}^{44}$ The present calculations predicts a pressure-induced phase transition at $0 \mathrm{~K}$ to occur at a considerably lower $\sim 6 \mathrm{GPa}$. However, a number of other investigations of the pressure induced phase transition have yielded results much closer to our predictions. Loa et al. performed angle-dispersive synchrotron x-ray powder diffraction measurements and found the transition pressure to be around $10.5 \mathrm{GPa} .{ }^{89}$ Alptekin et al. also measured the enthalpy of formation and predicted the transition pressure to be around $7 \mathrm{GPa},{ }^{90}$ while Pal et al. predicted the a transition pressure of $\sim 6.2 \mathrm{GPa}$ based on Raman measurements and first-principles DFT calculations. ${ }^{45}$ Our predictions are a good match for these results. Yu et al. ${ }^{91}$ predicted a $0 \mathrm{~K}$ transition pressure of $8 \mathrm{GPa}$ based on calculated enthalpies, which is again in line with the present calculations, and also estimated a transition temperature of $500 \mathrm{~K}$ at $4 \mathrm{GPa}$ using $a b$ initio molecular dynamics (AIMD). Our work suggests a lower transition temperature of $\sim 150 \mathrm{~K}$ at this pressure, but results in the same qualitative conclusion that the phase transition temperature is reduced under pressure. 

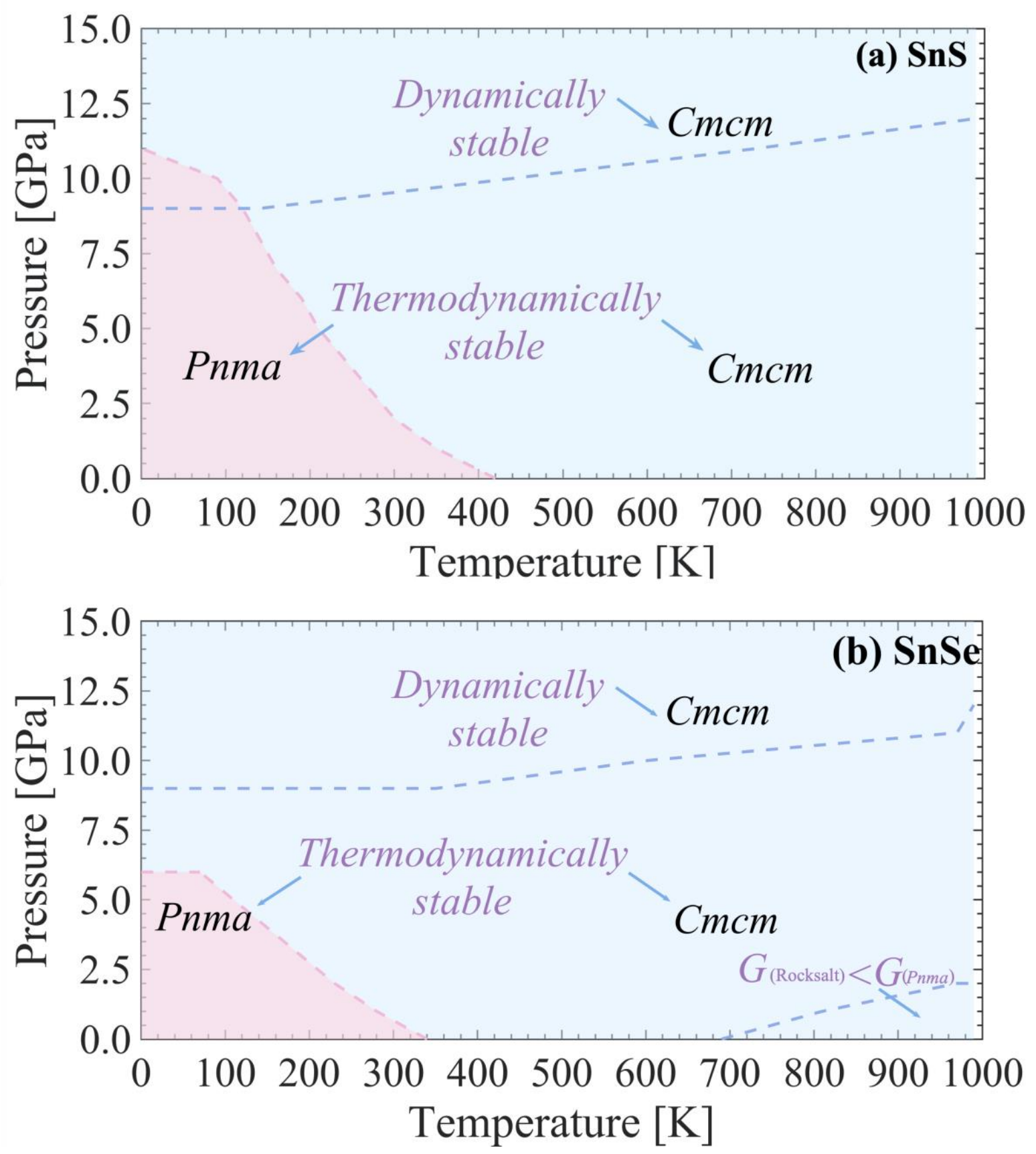

Figure 9. Calculated temperature-pressure phase diagrams of (a) $\mathrm{SnS}$ and (b) $\mathrm{SnSe}$, obtained based on the Gibbs energies calculated within the quasi-harmonic approximation. Shaded regions denote the most thermodynamically stable phases: pink - Pnma, blue - Cmcm. Contour lines outline regions where $\mathrm{Cmcm}$ is dynamically stable, and where rocksalt $\mathrm{SnSe}$ is more thermodynamically stable than Pnma SnSe and the Cmcm phase is dynamically unstable. 
A pressure-induced Pnma $\rightarrow \mathrm{Cmcm}$ phase transition has also been reported for SnS, albeit at higher pressures. Our QHA calculations suggest a transition to the $\mathrm{Cmcm}$ phase would occur around $11 \mathrm{GPa}$ at $0 \mathrm{~K}$, but calculations by Gashimzade et al. ${ }^{92}$ and Alptekin et al. ${ }^{69}$ predict 16 and 15 GPa respectively, although enthalpy calculations performed as part of the latter study predicted a much lower transition pressure of $4.5 \mathrm{GPa}$ at $0 \mathrm{~K}$. Nonetheless, the QHA calculations again make the same qualitative prediction that the transition pressure is likely to be higher in $\mathrm{SnS}$ than in SnSe.

Although the effect of renormalizing the imaginary modes in the $\mathrm{Cm} \mathrm{cm}$ phases appears to have a negligible impact on the on the Helmholtz and Gibbs free energy differences and hence the Pnma $\leftrightarrow \mathrm{Cmcm}$ phase-transition temperatures, we cannot rule out that anharmonic effects would have an impact on the predicted phase diagrams in Figure 9. Indeed, we would expect deviation from the QHA model at temperatures above $\sim 2 / 3$ the melting temperature $T_{\mathrm{m}} \cdot{ }^{93,94}$ However, as noted above, our finding that pressure stabilizes the $\mathrm{Cmcm}$ phase and reduces the transition temperature is in line with experiments and theoretical studies using other methods.

For comparison, we also investigated the simpler and less computationally-demanding quasi-harmonic Debye model, ${ }^{95}$ which used the $E / V$ curves and an approximate model of the phonon spectrum to make similar predictions to the QHA. We found that this model predicts $\mathrm{Cmcm} \mathrm{SnS}$ to be the most stable phase only above $30 \mathrm{GPa}$ and $850 \mathrm{~K}$, and $\mathrm{Cmcm}$ SnSe to be unstable at all the pressures and temperatures we tested. We therefore conclude that using the full phonon DoS at the harmonic and quasi-harmonic levels gives better predictions compared to experimental measurements. 
Finally, at the QHA level, issues with imaginary modes would only impact the boundaries of the region where the $\mathrm{Cmcm}$ phase is energetically stable but dynamically unstable. At high pressures, the $\mathrm{Cmcm}$ phase becomes dynamically stable, and rocksalt SnSe and the $\pi$-cubic phases of both monochalcogenides do not show imaginary modes in the dispersions. The latter implies that the observation that the two $\pi$-cubic phases do not appear on the phase diagram is correct within the QHA, which may be taken as evidence that the $\pi$-cubic phases are kinetically stabilized under the formation conditions.

\section{e. Structural Relationships Between Phases}

\section{i. The Pnma and Cmcm Phases}

Previous theoretical studies and experimental INS measurements have shown that the Pnma $\leftrightarrow \mathrm{Cmcm}$ phase transition is driven by condensation of a "soft" TO phonon mode of $\mathrm{Ag}_{\mathrm{g}}$ symmetry found at the Brillouin zone center in the Pnma phase and the $Y$ wavevector in the $\mathrm{Cmcm}$ phase. ${ }^{85}$ As noted previously, these soft modes manifest as negative eigenvalues in the dynamical matrix of the equilibrium structure, corresponding to imaginary harmonic frequencies, and indicate the structures to be local maximum on the structural PES. By "following" the eigenvectors corresponding to those modes, one can locate the nearest lower-energy stationary point, which may be a minimum or another maximum.

The atomic displacements $\boldsymbol{u}_{j l}$ along the mode eigenvector $\boldsymbol{W}_{\lambda}$ as a function of the normalmode coordinate (displacement amplitude) $Q_{\lambda}$ are given by: ${ }^{49}$

$$
\boldsymbol{u}_{j l}\left(Q_{\lambda}\right)=\frac{Q_{\lambda}}{\sqrt{N_{a} m_{j}}} \boldsymbol{w}_{\lambda} e^{i \boldsymbol{q} \cdot \boldsymbol{r}_{j l}}
$$


where we have replaced the wavevector and band index with a single index $\lambda$ for brevity. $N_{a}$ is the number of atoms in the supercell used to describe the vibration, which should be commensurate with the wavevector, $m_{j}$ is the atomic mass, $\boldsymbol{q}$ is the phonon wavevector and $\boldsymbol{r}_{j l}$ are the equilibrium positions. At small amplitudes the energy change $\Delta E$ with respect to the distortion amplitude is given by the usual harmonic relation, where $\omega$ corresponds to the harmonic frequency :63

$$
\Delta E=\frac{1}{2} \omega^{2} Q_{\lambda}{ }^{2}
$$

At larger amplitudes, the PES becomes anharmonic and typically resembles higher-order polynomials.

To investigate the link between the ground-state Pnma structure and the high-temperature $\mathrm{Cmcm}$ phase, the two imaginary modes at the $Y$ and $\Gamma$ wavevectors were mapped as a function of the corresponding normal-mode coordinates, using the ModeMap code,${ }^{63}$ to obtain a $1 \mathrm{D}$ PES along the two modes individually and a combined 2D PES where the structure is distorted along both modes simultaneously. The PES curves associated with $Y$ - and $\Gamma$-point imaginary modes in $\mathrm{Cmcm}$ $\mathrm{SnS}$, computed for a series of volumes including up to $6 \%$ compressions and $15 \%$ expansions about the equilibrium, are shown in Figure 10. Equivalent plots for SnSe are presented in Figure S9 in the Supporting Information.

The 1D plots show that both imaginary modes in $\mathrm{SnS}$ and SnSe are associated with a double-well PES. The mode at $Y$ has the deepest minima and leads to equivalent distorted Pnma structures, whereas the mode at $\Gamma$ has a steeper local curvature close to the $\mathrm{Cmcm}$ phase and thus a "more imaginary" harmonic frequency. The 2D PES shows the Cmcm phase to be a hilltop in the space spanned by the two imaginary modes. These plots confirm that the "true" minima lie along the $Y$-point mode, whereas the local minima along the $\Gamma$-point soft mode are saddle points. Under compression, the barriers from the minima to the $\mathrm{Cmcm}$ local maximum become smaller 
and eventually disappear, coinciding with the structure becoming dynamically stable under pressure. On the other hand, as the structure is expanded the 2D PES appears to become more symmetric.

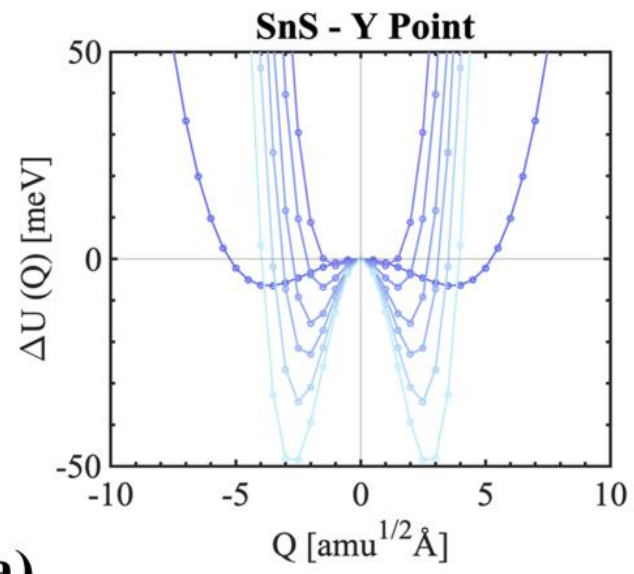

(a)
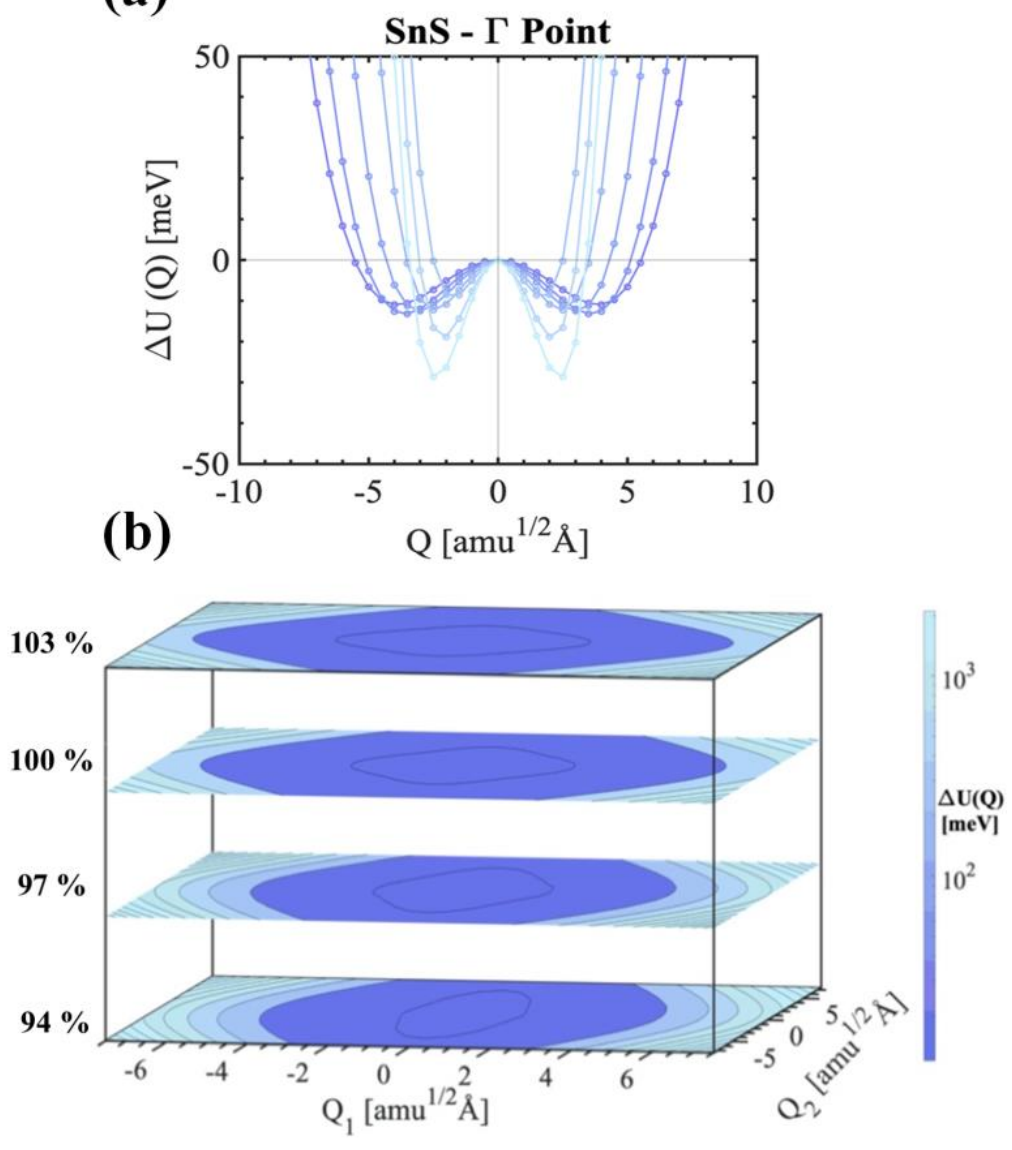

Figure 10. (a) Potential-energy surface (PES) along the two principal imaginary modes at the $Y$ and $\Gamma$ wavevectors in $\mathrm{Cmcm} \mathrm{SnS}$, evaluated for a series of volumes including up to $6 \%$ 
compressions and $15 \%$ expansions. (b) Two-dimensional PES spanned by both imaginary modes at four volumes ranging from $6 \%$ compressions to $3 \%$ expansions.

\section{ii. Rocksalt and $\pi$-Cubic Phases}

The mode mapping approach outlined above can be used to characterize the structural PES linking a local maximum with imaginary harmonic modes to the nearest local minima. However, the same method cannot be used to link the two cubic phases, since for SnSe both phases are dynamically-stable local minima. The $\pi$-cubic structure can be generated by a continuous distortion of a $2 \times 2 \times 2$ supercell of the eight-atom rocksalt conventional cell, so we instead performed transition-state searches using the climbing-image nudged elastic-band (CI-NEB) method.

The NEB method provides an efficient and accurate means of locating transition states. A series of structural snapshots ("images") are first defined which describe the reaction coordinate. Atoms in each image are connected to the equivalent atoms in adjacent images via harmonic strings ("elastic bands") with an associated force constant. The force acting on each atom has two components: the sum of the spring force projected along the path and the atomic force perpendicular to the path. ${ }^{96}$ The images are then optimized to minimize the force while maintaining equal spacing between neighboring images, thus refining the path to minimize the activation energy. The CI-NEB is a slight modification of the NEB that attempts to move the highest-energy image uphill to the saddle point, thereby providing a better estimate of the activation barrier. ${ }^{65}$

The energies along the pathways between the rocksalt and $\pi$-cubic phases of SnS and SnSe are shown in Figure 11. The structures of the initial rocksalt supercell, the transition state (highestenergy configuration along the path), and the final $\pi$-cubic structure are compared in Figure S11 in the Supporting Information. 

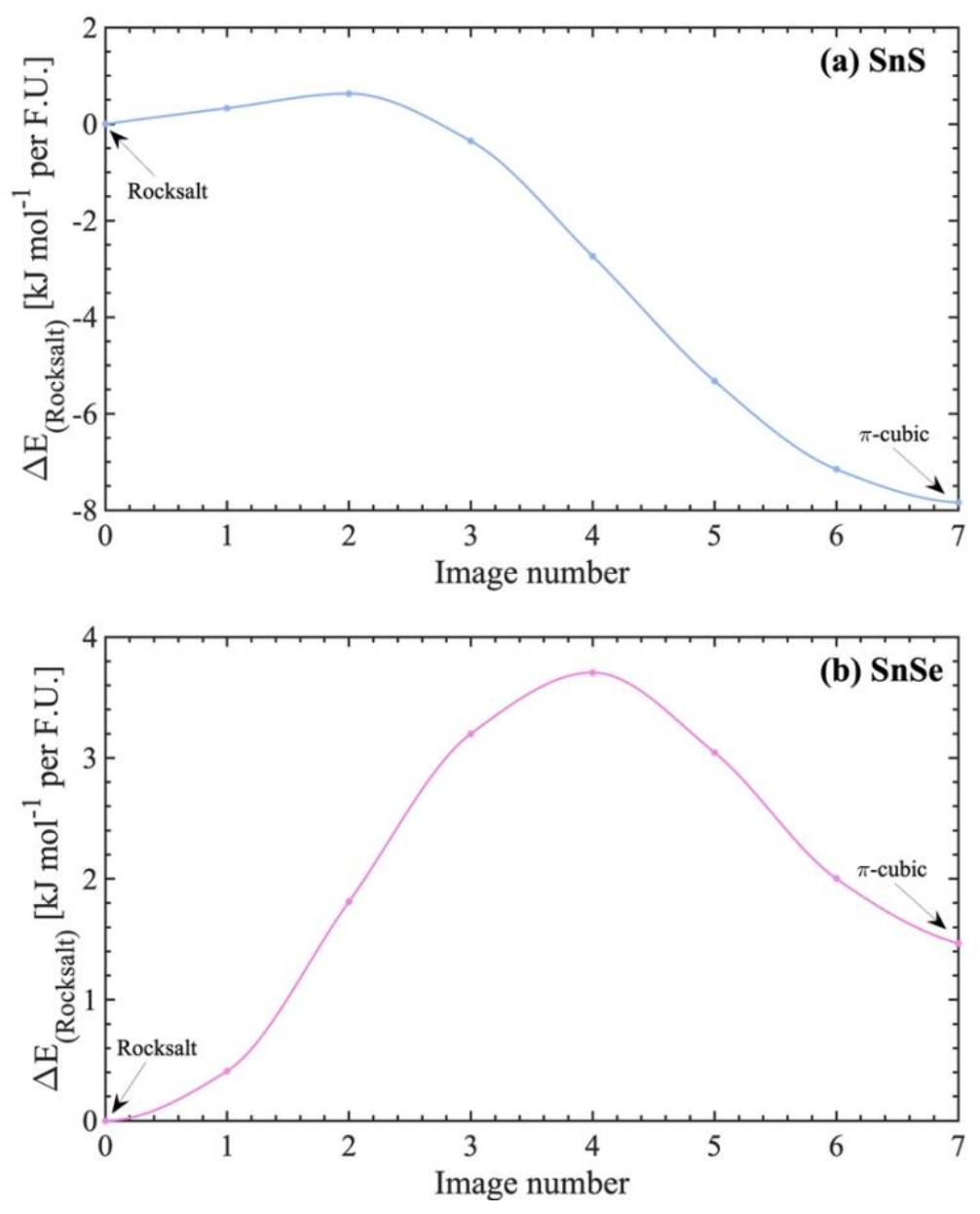

Figure 11. Difference in energy relative to the rocksalt phase as a function of reaction coordinate (“image number") between a 64-atom rocksalt supercell (Image 0) and the 64-atom $\pi$-cubic phase (Image 7) for (a) SnS and (b) SnSe. These profiles were obtained using the climbing-image nudged elastic band (CI-NEB) method to locate the transition states between the two phases.

The two sets of energy profiles show that there is a small activation barrier between the rocksalt and $\pi$-cubic phases in both $\mathrm{SnS}$ and $\mathrm{SnSe}$. From the rocksalt phase, the barriers are 0.63 and $3.71 \mathrm{~kJ} \mathrm{~mol}^{-1}$ per F.U. in $\mathrm{SnS}$ and SnSe, respectively, while the barriers from the $\pi$-cubic phases are 8.47 and $2.24 \mathrm{~kJ} \mathrm{~mol}^{-1}$ per F.U. respectively. If the transition to the orthorhombic phase 
proceeds via the rocksalt phase, the large barrier in $\pi$-cubic $\mathrm{SnS}$ may explain why this phase is kinetically stable once formed. ${ }^{21}$ Compared to $\mathrm{SnS}$, SnSe has a smaller activation barrier from the $\pi$-cubic phase and a larger barrier from the rocksalt phase. This is consistent with the previous findings on the relative stability of the rocksalt phase, i.e. that the rocksalt phase of SnSe is more stable than the rocksalt phase of SnS. In line with this, comparing the energy profiles shows that transition state (highest energy structure) occurs at the second image for SnS but at the fourth image for SnSe. The transition state for $\mathrm{SnS}$ is therefore more "rocksalt-like", whereas that for SnSe is more similar to the $\pi$-cubic phase.

\section{CONCLUSIONS}

In conclusion, we have performed a detailed first-principles investigation of the thermodynamic and dynamical stability of the four proposed phases of the tin monochalcogenides $\mathrm{SnS}$ and SnSe.

Assessment of the dynamical stability from the phonon DoS and dispersion curves shows that the Pnma and $\pi$-cubic phases of both systems are energy minima. The $\mathrm{Cmcm}$ phase is dynamically unstable, but becomes stable under a $\sim 12 \%$ compression of the lattice volume. Whereas rocksalt SnS shows an imaginary mode at the $X$ wavevector, which also gradually hardens and disappears under compression, the corresponding selenide phase is found to be dynamically stable.

Comparison of the Helmholtz and Gibbs free energies at ambient pressure, computed within the harmonic and quasi-harmonic approximations, correctly predicts the thermal phase transition between the Pnma and $\mathrm{Cmcm}$ phases, albeit at lower temperatures than those reported experimentally. The $\pi$-cubic phase of both $\mathrm{SnS}$ and SnSe remains metastable up to $1000 \mathrm{~K}$. 
Rocksalt $\mathrm{SnSe}$ is predicted to be significantly more stable than rocksalt $\mathrm{SnS}$ and is closer to the Pnma phase than the $\pi$-cubic structure. Based on the Gibbs free energy, rocksalt SnSe becomes lower in energy than the Pnma phase at elevated temperature, and may therefore be observed at higher temperatures. 2D temperature-pressure phase diagrams computed for temperature ranges of 0-1000 K and pressure ranges of 0-15 GPa identify only the orthorhombic Pnma and Cmcm structures as the phases with the lowest Gibbs free energies. For both $\mathrm{SnS}$ and SnSe the transition temperature from the Pnma to the $\mathrm{Cmcm}$ phase is predicted to decrease under pressure, and above $11 \mathrm{GPa}$ and $7 \mathrm{GPa}$ for $\mathrm{SnS}$ and $\mathrm{SnSe}$, respectively, the $\mathrm{Cmcm}$ structure becomes the most thermodynamically stable phase from $0-1000 \mathrm{~K}$. The $\mathrm{Cmcm}$ phase is predicted to be both dynamically and thermodynamically stable above $11 \mathrm{GPa}$ and $9 \mathrm{GPa}$ for $\mathrm{SnS}$ and SnSe. At temperatures between $\sim 700-1000 \mathrm{~K}$ and pressures of $0-2 \mathrm{GPa}$, rocksalt $\mathrm{SnSe}$ is more thermodynamically stable than Pnma SnSe and the Cmcm phase is dynamically unstable, which suggests the rocksalt phase may be observed as a local structure. The QHA calculations also predict that the $\pi$-cubic phase does not appear on either phase diagram, which suggests that it is formed by virtue of being kinetically stabilized under the reaction conditions.

First-principles phonon mode mapping and transition-state searches using the climbingimage nudged elastic band method were used to find the structural links between the orthorhombic and cubic phases. The two principal soft modes in the $\mathrm{Cmcm}$ phase are both associated with a double-well PES and identify the $\mathrm{Cmcm}$ as a hilltop connecting two Pnma energy minima. The energy barrier is enlarged under expansion (temperature) and reduced under compression (pressure). The rocksalt and $\pi$-cubic phases of $\mathrm{SnS}$ and $\mathrm{SnSe}$ are separated by small activation barriers of $0.63 / 3.71 \mathrm{~kJ} \mathrm{~mol}^{-1}$ per F.U., respectively, from the $\pi$-cubic to rocksalt phases, and 
$8.47 / 2.24 \mathrm{~kJ} \mathrm{~mol}^{-1}$ per F.U. in the reverse direction. The large activation barrier from $\pi$-cubic $\mathrm{SnS}$ may explain why this phase is reasonably stable once formed.

Together, these results provide a comprehensive assessment of the relative stabilities of the monochalcogenide phases, and we hope they will serve as a useful reference point for experimental synthesis and characterization. They also highlight some interesting possibilities for using pressure, for example by materials engineering or epitaxial growth, to fine-tune the material properties. In particular, our calculations suggest that this may provide some level of control over the Pnma to $\mathrm{Cmcm}$ phase transition and may allow rocksalt $\mathrm{SnS}$ to be stabilized. Given that the high thermoelectric performance of bulk $\mathrm{SnSe}$ is associated with the inherent anharmonicity and favorable electrical properties of the $\mathrm{Cmcm}$ phase, and that the rocksalt phases have been identified as interesting optoelectronic materials in their own right, this may provide an interesting route to further applications of these systems.

\section{ASSOCIATED CONTENT}

\section{Supporting Information}

The Supporting Information is available free of charge on the ACS Publications website at DOI:

xxx.yy/ZZZZ

Images of the optimized structures of $\mathrm{SnS}$; $\boldsymbol{k}$-point convergence tests for phonon calculations on the $\pi$-cubic phases of $\mathrm{SnS}$ and $\mathrm{SnSe}$; calculated electronic atom- and orbital-projected density of states (DoS) curves for the rocksalt phases of SnS and SnSe; calculated electronic bandgaps of the four phases of $\mathrm{SnS}$ and $\mathrm{SnSe}$; effective renormalised frequencies of the two imaginary modes of $\mathrm{SnS}$ and $\mathrm{SnSe} C \mathrm{mcm}$ as a function of 
temperature; anharmonic double-well potentials along the two imaginary harmonic modes of $\mathrm{Cmcm} \mathrm{SnS}$ and $\mathrm{SnSe}$ as a function of the corresponding normal-mode coordinates showing the eigenvalues obtained by solving a 1D Schrödinger equation for the potential; phonon dispersion and density of states curves for $\mathrm{SnS}$ and $\mathrm{SnSe}$ at a series of volumes from $27 \%$ compressions to $15 \%$ expansions; Gibbs energy differences between the Pnma and $\mathrm{Cmcm}$ phases of $\mathrm{SnS}$ and $\mathrm{SnSe}$ before and after renormalization; optimized structures of Pnma and Cmcm SnS and SnSe under pressure; calculated potential-energy surfaces associated with the principal imaginary modes in $\mathrm{Cmcm} \mathrm{SnS}$ and $\mathrm{SnSe}$, computed for a series of volumes from $6 \%$ compressions to $15 \%$ expansions; structures of SnSe obtained by mapping the $\Gamma$-point imaginary modes; structures of $\mathrm{SnS}$ and SnSe obtained from the transition-state searches using the CI-NEB method; plots of the cell volume, bulk modulus and thermodynamic Grüneisen parameters as a function of temperature and at ambient pressure obtained using the quasi-harmonic approximation (PDF).

\section{Data Repository}

Raw data from this study including optimized structures and output from the quasi-harmonic lattice dynamics calculations is available to download for free from an online repository at xxx.yy/ZZZZ

\section{AUTHOR INFORMATION}

\section{Corresponding Author}

*E-mail: jonathan.skelton@manchester.ac.uk 


\section{ORCID}

Ioanna Pallikara: 0000-0003-4123-6135

Jonathan M. Skelton: 0000-0002-0395-1202

\section{Funding Sources}

JS is supported by a UK Research and Innovation Future Leaders Fellowship (MR/T043121/1). Our work made use of the UK Archer HPC facility via the UK Materials Chemistry Consortium, which is funded by the UK Engineering and Physical Sciences Research Council (EPSRC; grant nos. EP/L000202, EP/R029431, EP/T022213), and the Beskow service at the KTH PDC facility in Sweden via the PRACE award 15DECI0404/QTProp.

\section{ACKNOWLEDGMENTS}

IP is grateful to the University of Manchester for the support of a PhD studentship. JS is supported by a UK Research and Innovation Future Leaders Fellowship (MR/T043121/1) and previously held a University of Manchester Presidential Fellowship. The calculations reported in this work made use of three high-performance computing (HPC) systems. We used the UK Archer HPC facility via our membership of the UK Materials Chemistry Consortium, which is funded by the UK Engineering and Physical Sciences Research Council (EPSRC; grant nos. EP/L000202, EP/R029431 and EP/T022213). We also made use of the Beskow HPC service at the KTH PDC facility in Sweden via the PRACE award 15DECI0404/QTProp. Some of our calculations were also performed on the University of Manchester Computational Shared Facility, which is maintained by UoM Research IT. 


\section{ABBREVIATIONS}

PV - photovoltaic; DFT - density-functional theory; SIBs - sodium-ion batteries; LIBs - lithiumion batteries; TCIs - topological crystalline insulators; DoS - (phonon) density of states; EoS equation of state; VASP - Vienna Ab initio Simulation Package; PAW - projector augmented-wave (method); CI-NEB - climbing-image nudged elastic band (method).

\section{REFERENCES}

(1) Shi, W.; Gao, M.; Wei, J.; Gao, J.; Fan, C.; Ashalley, E.; Li, H.; Wang, Z. Tin Selenide (SnSe): Growth, Properties, and Applications. Adv. Sci. 2018, 5 (4), 1-22. https://doi.org/10.1002/advs.201700602.

(2) Koteeswara Reddy, N.; Devika, M.; Gopal, E. S. R. Review on Tin (II) Sulfide (SnS) Material: Synthesis, Properties, and Applications. Crit. Rev. Solid State Mater. Sci. 2015, 40 (6), 359-398. https://doi.org/10.1080/10408436.2015.1053601.

(3) Xu, Y.; Al-Salim, N.; Hodgkiss, J. M.; Tilley, R. D. Solution Synthesis and Optical Properties of SNTE Nanocrystals. Cryst. Growth Des. 2011, 11 (7), 2721-2723. https://doi.org/10.1021/cg200660y.

(4) Wang, C.; Li, Y. D.; Zhang, G. H.; Zhuang, J.; Shen, G. Q. Synthesis of SnSe in Various Alkaline Media under Mild Conditions. Inorg. Chem. 2000, 39 (19), 4237-4239. https://doi.org/10.1021/ic991332b.

(5) Parenteau, M.; Carlone, C. Influence of Temperature and Pressure on the Electronic Transitions in SnS and SnSe Semiconductors. Phys. Rev. B 1990, 41 (8), 5227-5234. https://doi.org/10.1103/PhysRevB.41.5227.

(6) Wang, M. X.; Yue, G. H.; Lin, Y. D.; Wen, X.; Peng, D. L.; Geng, Z. R. Optical Properties and Photovoltaic Application of the SnS Quasi-One-Dimensional Nanostructures. Nano- 
Micro Lett. 2013, 5 (1), 1-6. https://doi.org/10.3786/nml.v5i1.p1-6.

(7) Zhou, X.; Zhang, Q.; Gan, L.; Li, H.; Xiong, J.; Zhai, T. Booming Development of Group IV-VI Semiconductors: Fresh Blood of 2D Family. Adv. Sci. 2016, 3 (12). https://doi.org/10.1002/advs.201600177.

(8) Minnam Reddy, V. R.; Gedi, S.; Pejjai, B.; Park, C. Perspectives on SnSe-Based Thin Film Solar Cells: A Comprehensive Review. J. Mater. Sci. Mater. Electron. 2016, 27 (6), 54915508. https://doi.org/10.1007/s10854-016-4563-9.

(9) Walsh, A.; Watson, G. W. Influence of the Anion on Lone Pair Formation in $\mathrm{Sn}(\mathrm{II})$ Monochalcogenides: A DFT Study. J. Phys. Chem. B 2005, 109 (40), 18868-18875. https://doi.org/10.1021/jp051822r.

(10) An, C.; Tang, K.; Jin, Y.; Liu, Q.; Chen, X.; Qian, Y. Shape-Selected Synthesis of Nanocrystalline SnS in Different Alkaline Media. J. Cryst. Growth 2003, 252 (4), 581-586. https://doi.org/10.1016/S0022-0248(03)00961-8.

(11) Timofeev, Y. A.; Vinogradov, B. V; Begoulev, V. B. Superconductivity of Tin Selenide at Pressures up to 70 GPa. Phys. Solid State 1997, 39 (2), 207. https://doi.org/10.1134/1.1130136.

(12) Odani, A.; Nimberger, A.; Markovsky, B.; Sominski, E.; Levi, E.; Kumar, V. G.; Motiei, M.; Gedanken, A.; Dan, P.; Aurbach, D. Development and Testing of Nanomaterials for Rechargeable Lithium Batteries. J. Power Sources 2003, 119-121, 517-521. https://doi.org/10.1016/S0378-7753(03)00276-3.

(13) Takeuchi, K.; Ichimura, M.; Arai, E.; Yamazaki, Y. SnS Thin Films Fabricated by Pulsed and Normal Electrochemical Deposition. Sol. Energy Mater. Sol. Cells 2003, 75 (3-4), 427432. https://doi.org/10.1016/S0927-0248(02)00192-7. 
(14) Burton, L. A.; Walsh, A. Phase Stability of the Earth-Abundant Tin Sulfides SnS, SnS2, and Sn2S3. J. Phys. Chem. C 2012, $116 \quad$ (45), 24262-24267. https://doi.org/10.1021/jp309154s.

(15) Peter, L. M. Towards Sustainable Photovoltaics: The Search for New Materials. Philos. Trans. R. Soc. A Math. Phys. Eng. Sci. 2011, 369 (1942), 1840-1856. https://doi.org/10.1098/rsta.2010.0348.

(16) Avellaneda, D.; Nair, M. T. S.; Nair, P. K. Polymorphic Tin Sulfide Thin Films of Zinc Blende and Orthorhombic Structures by Chemical Deposition. J. Electrochem. Soc. 2008, 155 (7), D517. https://doi.org/10.1149/1.2917198.

(17) Abutbul, R. E.; Segev, E.; Samuha, S.; Zeiri, L.; Ezersky, V.; Makov, G.; Golan, Y. A New Nanocrystalline Binary Phase: Synthesis and Properties of Cubic Tin Monoselenide. CrystEngComm 2016, 18 (11), 1918-1923. https://doi.org/10.1039/c5ce02437d.

(18) Abutbul, R. E.; Garcia-Angelmo, A. R.; Burshtein, Z.; Nair, M. T. S.; Nair, P. K.; Golan, Y. Crystal Structure of a Large Cubic Tin Monosulfide Polymorph: An Unraveled Puzzle. CrystEngComm 2016, 18 (27), 5188-5194. https://doi.org/10.1039/c6ce00647g.

(19) Rabkin, A.; Samuha, S.; Abutbul, R. E.; Ezersky, V.; Meshi, L.; Golan, Y. New Nanocrystalline Materials: A Previously Unknown Simple Cubic Phase in the SnS Binary System. Nano Lett. 2015, 15 (3), 2174-2179. https://doi.org/10.1021/acs.nanolett.5b00209.

(20) Skelton, J. M.; Burton, L. A.; Oba, F.; Walsh, A. Chemical and Lattice Stability of the Tin Sulfides. J. Phys. Chem. C 2017, $121 \quad$ (12), 6446-6454. https://doi.org/10.1021/acs.jpcc.6b12581.

(21) Skelton, J. M.; Burton, L. A.; Oba, F.; Walsh, A. Metastable Cubic Tin Sulfide: A Novel Phonon-Stable Chiral Semiconductor. APL Mater. 2017, 5 
https://doi.org/10.1063/1.4977868.

(22) Mariano, A. N.; Chopra, K. L. Polymorphism in Some IV-VI Compounds Induced by High Pressure and Thin-Film Epitaxial Growth. Appl. Phys. Lett. 1967, 10, 282-284.

(23) Wang, D.; He, W.; Chang, C.; Wang, G.; Wang, J.; Zhao, L. D. Thermoelectric Transport Properties of Rock-Salt SnSe: First-Principles Investigation. J. Mater. Chem. C 2018, 6 (44), 12016-12022. https://doi.org/10.1039/c8tc01314d.

(24) Ramakrishna Reddy, K. T.; Koteswara Reddy, N.; Miles, R. W. Photovoltaic Properties of SnS Based Solar Cells. Sol. Energy Mater. Sol. Cells 2006, 90 (18-19), 3041-3046. https://doi.org/10.1016/j.solmat.2006.06.012.

(25) Banai, R. E.; Burton, L. A.; Choi, S. G.; Hofherr, F.; Sorgenfrei, T.; Walsh, A.; To, B.; Cröll, A.; Brownson, J. R. S. Ellipsometric Characterization and Density-Functional Theory Analysis of Anisotropic Optical Properties of Single-Crystal $\alpha$-SnS. J. Appl. Phys. 2014, 116 (1), 013511. https://doi.org/10.1063/1.4886915.

(26) Scragg, J. J.; Wätjen, J. T.; Edoff, M.; Ericson, T.; Kubart, T.; Platzer-Björkman, C. A Detrimental Reaction at the Molybdenum Back Contact in $\mathrm{Cu} 2 \mathrm{ZnSn}(\mathrm{S}, \mathrm{Se}) 4$ Thin-Film Solar Cells. J. Am. Chem. Soc. 2012, 134 (47), 19330-19333. https://doi.org/10.1021/ja308862n.

(27) Nair, M. T. S.; Barrios-Salgado, E.; García, A. R.; Aragón-Silva, M. R.; Campos, J.; Nair, P. K. Chemically Deposited Tin Chalcogenides as Absorbers in Thin Film Solar Cells. ECS Trans. 2019, 41 (4), 177-183. https://doi.org/10.1149/1.3628623.

(28) Momma, K.; Izumi, F. VESTA3 for Three-Dimensional Visualization of Crystal, Volumetric and Morphology Data. J. Appl. Crystallogr. 2011, 44 (6), 1272-1276. https://doi.org/10.1107/S0021889811038970. 
(29) Nair, P. K.; Garcia-Angelmo, A. R.; Nair, M. T. S. Cubic and Orthorhombic SnS Thin-Film Absorbers for Tin Sulfide Solar Cells. Phys. status solidi 2016, 213 (1), 170-177. https://doi.org/10.1002/pssa.201532426.

(30) Sist, M.; Zhang, J.; Brummerstedt Iversen, B. Crystal Structure and Phase Transition of Thermoelectric SnSe. Acta Crystallogr. Sect. B Struct. Sci. Cryst. Eng. Mater. 2016, 72 (3), 310-316. https://doi.org/10.1107/S2052520616003334.

(31) Skipidarov, S.; Nikitin, M. Novel Thermoelectric Materials and Device Design Concepts; Skipidarov, S., Nikitin, M., Eds.; Springer International Publishing: Switzerland, 2019. https://doi.org/10.1007/978-3-030-12057-3.

(32) Tan, Q.; Zhao, L. D.; Li, J. F.; Wu, C. F.; Wei, T. R.; Xing, Z. B.; Kanatzidis, M. G. Thermoelectrics with Earth Abundant Elements: Low Thermal Conductivity and High Thermopower in Doped SnS. J. Mater. Chem. A 2014, 2 (41), 17302-17306. https://doi.org/10.1039/c4ta04462b.

(33) Sun, B. Z.; Ma, Z.; He, C.; Wu, K. Enhanced Thermoelectric Performance of Layered SnS Crystals: The Synergetic Effect of Temperature and Carrier Concentration. RSC Adv. 2015, 5 (69), 56382-56390. https://doi.org/10.1039/c5ra06134b.

(34) Xie, Y.; Zhou, Y.; Gong, X. G. The Intrinsic Low Lattice Thermal Conductivity in the Rock Salt SnSe. Comput. Mater. Sci. $\quad$ 2018, $\quad$ 148, 54-59. https://doi.org/10.1016/j.commatsci.2018.01.045.

(35) Jia, H.; Dirican, M.; Sun, N.; Chen, C.; Zhu, P.; Yan, C.; Dong, X.; Du, Z.; Guo, J.; Karaduman, Y.; Wang, J.; Tang, F.; Tao, J.; Zhang, X. SnS Hollow Nanofibers as Anode Materials for Sodium-Ion Batteries with High Capacity and Ultra-Long Cycling Stability. Chem. Commun. 2019, 55 (4), 505-508. https://doi.org/10.1039/C8CC07332E. 
(36) Balli, B.; Şavk, A.; Şen, F. 5 - Graphene and Polymer Composites for Supercapacitor Applications. In Woodhead Publishing Series in Composites Science and Engineering;

Khan, A., Jawaid, M., Inamuddin, Asiri, A. M., Eds.; Woodhead Publishing Series in Composites Science and Engineering; Woodhead Publishing, 2019; pp 123-151. https://doi.org/https://doi.org/10.1016/B978-0-08-102509-3.00005-5.

(37) Wang, X.; Liu, B.; Xiang, Q.; Wang, Q.; Hou, X.; Chen, D.; Shen, G. Spray-Painted BinderFree SnSe Electrodes for High-Performance Energy-Storage Devices. ChemSusChem 2014, 7 (1), 308-313. https://doi.org/10.1002/cssc.201300241.

(38) Patil, A. M. Electrochemical Supercapacitor Properties of SnS Thin Films Deposited by Low-Cost Chemical Bath Deposition Route. Int. J. Eng. Res. Sci. Technol. 2017, 10 (1), 914-922.

(39) Fu, L. Topological Crystalline Insulators. Phys. Rev. Lett. 2011, 106 (10), 1-4. https://doi.org/10.1103/PhysRevLett.106.106802.

(40) Sun, Y.; Zhong, Z.; Shirakawa, T.; Franchini, C.; Li, D.; Li, Y.; Yunoki, S.; Chen, X. Q. Rocksalt SnS and SnSe: Native Topological Crystalline Insulators. Phys. Rev. B - Condens. Matter Mater. $\quad$ Phys. $\quad$ 2013, $\quad 88 \quad$ (23), $\quad$ 235122-235122. https://doi.org/10.1103/PhysRevB.88.235122.

(41) Li, C. W.; Hong, J.; May, A. F.; Bansal, D.; Chi, S.; Hong, T.; Ehlers, G.; Delaire, O. Orbitally Driven Giant Phonon Anharmonicity in SnSe. Nat. Phys. 2015, 11 (12), 10631069. https://doi.org/10.1038/nphys3492.

(42) Aseginolaza, U.; Bianco, R.; Monacelli, L.; Paulatto, L.; Calandra, M.; Mauri, F.; Bergara, A.; Errea, I. Phonon Collapse and Second-Order Phase Transition in Thermoelectric SnSe. Phys. Rev. Lett. 2019, 122 (7), 1-6. https://doi.org/10.1103/PhysRevLett.122.075901. 
(43) Aseginolaza, U.; Bianco, R.; Monacelli, L.; Paulatto, L.; Calandra, M.; Mauri, F.; Bergara, A.; Errea, I. Strong Anharmonicity and High Thermoelectric Efficiency in HighTemperature SnS from First Principles. Phys. Rev. B 2019, 100 (21), 214307. https://doi.org/10.1103/PhysRevB.100.214307.

(44) Yang, L. T.; Ding, L. P.; Shao, P.; Tiandong, Y. H.; Zhao, Z. L.; Zhang, F. H.; Lu, C. Influence of Pressure on Phase Transition, Electronic and Thermoelectric Properties of SnSe. J. Alloys Compd. 2021, 853, 157362. https://doi.org/10.1016/j.jallcom.2020.157362.

Pal, S.; Arora, R.; Roychowdhury, S.; Harnagea, L.; Saurabh, K.; Shenoy, S.; Muthu, D. V. S.; Biswas, K.; Waghmare, U. V.; Sood, A. K. Pressure-Induced Phase Transitions in the Topological Crystalline Insulator SnTe and Its Comparison with Semiconducting SnSe: Raman and First-Principles Studies. Phys. Rev. B 2020, 101 (15), 1-10. https://doi.org/10.1103/PhysRevB.101.155202.

(46) Liu, C. M.; Xu, C.; Duan, M. Y. Structural, Thermodynamic, Elastic, and Electronic Properties of $\alpha-S n S$ at High Pressure from First-Principles Investigations. Zeitschrift fur Naturforsch. - Sect. A J. Phys. Sci. 2015, 70 (11), 949-960. https://doi.org/10.1515/zna2015-0293.

(47) Togo, A.; Tanaka, I. First Principles Phonon Calculations in Materials Science. Scr. Mater. 2015, 108, 1-5. https://doi.org/10.1016/j.scriptamat.2015.07.021.

(48) Venkataraman, G.; Feldkamp, L. A.; Sahni, V. C. Dynamics of Perfect Crystals; MIT press: USA, 1975.

(49) Nasrollahi, S. H.; Vvedensky, D. D. Local Normal Modes and Lattice Dynamics. J. Appl. Phys. 2018, 124 (4), 045102. https://doi.org/10.1063/1.5034437.

(50) Dove, M. T. Structure and Dynamics — An Atomic View of Materials. Mater. Today 2003, 
6 (6), 59. https://doi.org/10.1016/s1369-7021(03)00639-4.

(51) Birch, F. Finite Elastic Strain of Cubic Crystals. Phys. Rev. 1947, 71 (11), 809-824. https://doi.org/10.1103/PhysRev.71.809.

(52) Kresse, G. Ab Initio Molecular Dynamics for Liquid Metals. J. Non. Cryst. Solids 1995, 192-193, 222-229. https://doi.org/10.1016/0022-3093(95)00355-X.

(53) Perdew, J. P.; Ruzsinszky, A.; Csonka, G. I.; Vydrov, O. A.; Scuseria, G. E.; Constantin, L. A.; Zhou, X.; Burke, K. Restoring the Density-Gradient Expansion for Exchange in Solids and Surfaces. Phys. Rev. Lett. 2008, $100 \quad$ (13), 136406. https://doi.org/10.1103/PhysRevLett.100.136406.

(54) Grimme, S.; Antony, J.; Ehrlich, S.; Krieg, H. A Consistent and Accurate Ab Initio Parametrization of Density Functional Dispersion Correction (DFT-D) for the 94 Elements H-Pu. J. Chem. Phys. 2010, 132 (15), 154104-undefined. https://doi.org/10.1063/1.3382344.

(55) Liu, H.; Chang, L. L. Y. Phase Relations in Systems of Tin Chalcogenides. J. Alloys Compd. 1992, 185 (1), 183-190. https://doi.org/10.1016/0925-8388(92)90566-R.

(56) Bilenkii, B. F.; Mikolaichuk, A. G.; Freik, D. M. Struktur Und Optische Eigenschaften von Epitaxialen SnTe-, SnSe- Und SnS-Schichten. Phys. status solidi 1968, 28 (1), K5-K7. https://doi.org/10.1002/pssb.19680280146.

(57) Jain, A.; Ong, S. P.; Hautier, G.; Chen, W.; Richards, W. D.; Dacek, S.; Cholia, S.; Gunter, D.; Skinner, D.; Ceder, G.; Persson, K. A. Commentary: The Materials Project: A Materials Genome Approach to Accelerating Materials Innovation. APL Mater. 2013, 1 (1), 011002. https://doi.org/10.1063/1.4812323.

(58) Kresse, G.; Joubert, D. From Ultrasoft Pseudopotentials to the Projector Augmented-Wave 
Method. Phys. Rev. B Condens. Matter Mater. Phys. 1999, 59, 1758-1775.

(59) Wang, V.; Xu, N.; Liu, J. C.; Tang, G.; Geng, W.-T. VASPKIT: A User-Friendly Interface Facilitating High-Throughput Computing and Analysis Using VASP Code. 2019.

(60) M Ganose, A.; J Jackson, A.; O Scanlon, D. Sumo: Command-Line Tools for Plotting and Analysis of Periodic Ab Initio Calculations. J. Open Source Softw. 2018, 3 (28), 717. https://doi.org/10.21105/joss.00717.

(61) Parlinski, K.; Li, Z. Q.; Kawazoe, Y. First-Principles Determination of the Soft Mode in Cubic ZrO2. Phys. Rev. Lett. 1997, 78, 4063-4066.

(62) Monkhorst, H. J.; Pack, J. D. Special Points for Brillouin-Zone Integrations. Phys. Rev. B 1976, 13 (12), 5188-5192. https://doi.org/10.1103/PhysRevB.13.5188.

(63) Skelton, J. M.; Burton, L. A.; Parker, S. C.; Walsh, A.; Kim, C.-E.; Soon, A.; Buckeridge, J.; Sokol, A. A.; Catlow, C. R. A.; Togo, A.; Tanaka, I. Anharmonicity in the HighTemperature Cmcm Phase of SnSe: Soft Modes and Three-Phonon Interactions. Phys. Rev. Lett. 2016, 117 (7), 075502. https://doi.org/10.1103/PhysRevLett.117.075502.

(64) Buckeridge, J.; Fahy, S. Mobility in Gated GaNxAs1-x Heterostructures as a Probe of Nitrogen-Related Electronic States. Phys. Rev. B - Condens. Matter Mater. Phys. 2011, 84 (14), 1-19. https://doi.org/10.1103/PhysRevB.84.144120.

(65) Henkelman, G.; Uberuaga, B. P.; Jónsson, H. Climbing Image Nudged Elastic Band Method for Finding Saddle Points and Minimum Energy Paths. J. Chem. Phys. 2000, 113 (22), 9901-9904. https://doi.org/10.1063/1.1329672.

(66) Zhao, L. D.; Lo, S. H.; Zhang, Y.; Sun, H.; Tan, G.; Uher, C.; Wolverton, C.; Dravid, V. P.; Kanatzidis, M. G. Ultralow Thermal Conductivity and High Thermoelectric Figure of Merit in SnSe Crystals. Nature 2014, 508 (7496), 373-377. https://doi.org/10.1038/nature13184. 
(67) Hofmann, W. Ergebnisse Der Strukturbestimmung Komplexer Sulfide. Zeitschrift für Krist. - Cryst. Mater. 2014, 92 (1-6), 161-undefined. https://doi.org/10.1524/zkri.1935.92.1.161.

(68) Skelton, J. M.; Tiana, D.; Parker, S. C.; Togo, A.; Tanaka, I.; Walsh, A. Influence of the Exchange-Correlation Functional on the Quasi-Harmonic Lattice Dynamics of II-VI Semiconductors. J. Chem. Phys. 2015, 143 (6). https://doi.org/10.1063/1.4928058.

(69) Alptekin, S.; Durandurdu, M. Formation of a Cmcm Phase in SnS at High Pressure; an Ab Initio Constant Pressure Study. Solid State Commun. 2010, 150 (17-18), 870-874. https://doi.org/10.1016/j.ssc.2010.02.002.

(70) Wiedemeier, H.; Schnering, H. G. Von. Refinement of the Structures of GeS, GeSe, SnS and SnSe. Zeitschrift fur Krist. - New Cryst. Struct. 1978, 148 (3-4), 295-303. https://doi.org/10.1524/zkri.1978.148.3-4.295.

(71) Ahmet, I. Y.; Hill, M. S.; Johnson, A. L.; Peter, L. M. Polymorph-Selective Deposition of High Purity SnS Thin Films from a Single Source Precursor. Chem. Mater. 2015, 27 (22), 7680-7688. https://doi.org/10.1021/acs.chemmater.5b03220.

(72) von Schnering, H. G.; Wiedemeier, H. The High Temperature Structure of $\beta$-SnS and $\beta$ SnSe and the B16-to-B33 Type A-Transition Path. Zeitschrift fur Krist. - New Cryst. Struct. 1981, 156(1-2), 143-150. https://doi.org/10.1524/zkri.1981.156.1-2.143.

(73) Lu, Y.; Zheng, F. W.; Yang, Y.; Zhang, P.; Zhang, D. B. Phase Stabilities of Cmcm and Pnma SnSe Studied by Phonon Quasiparticle Approach. Phys. Rev. B 2019, 100 (5). https://doi.org/10.1103/PhysRevB.100.054304.

(74) Rehman, S. U.; Butt, F. K.; Hayat, F.; Ul Haq, B.; Tariq, Z.; Aleem, F.; Li, C. An Insight into a Novel Cubic Phase SnSe for Prospective Applications in Optoelectronics and Clean Energy Devices. J. Alloys Compd. 2018, 733, 22-32. 
https://doi.org/10.1016/j.jallcom.2017.10.192.

(75) Rehman, S. ur; Butt, F. K.; Tariq, Z.; Hayat, F.; Gilani, R.; Aleem, F. Pressure Induced Structural and Optical Properties of Cubic Phase SnSe: An Investigation for the Infrared/Mid-Infrared Optoelectronic Devices. J. Alloys Compd. 2017, 695, 194-201. https://doi.org/10.1016/j.jallcom.2016.10.120.

(76) Jones, L. A. H.; Linhart, W. M.; Fleck, N.; Swallow, J. E. N.; Murgatroyd, P. A. E.; Shiel, H.; Featherstone, T. J.; Smiles, M. J.; Thakur, P. K.; Lee, T. L.; Hardwick, L. J.; Alaria, J.; Jäckel, F.; Kudrawiec, R.; Burton, L. A.; Walsh, A.; Skelton, J. M.; Veal, T. D.; Dhanak, V. R. Sn 5s2 Lone Pairs and the Electronic Structure of Tin Sulphides: A Photoreflectance, High-Energy Photoemission, and Theoretical Investigation. Phys. Rev. Mater. 2020, 4 (7), 74602. https://doi.org/10.1103/PhysRevMaterials.4.074602.

(77) Shaposhnikov, V. L.; Krivosheeva, A. V; Borisenko, V. E.; Lazzari, J. Structure, Electronic and Optical Properties of Tin Sulfide. ScienceJet 2012, No. Table 1, 1-4.

(78) Valiukonis, G.; Guseinova, D. A.; Krivaite, G.; Sileika, A. Optical Spectra and Energy Band Structure of Layer Type A(IV)B(VI) Compounds. Phys. Status Solidi 1986, 135 (2), 299 307. https://doi.org/https://doi.org/10.1002/pssb.2221350130.

(79) Abutbul, R. E.; Segev, E.; Zeiri, L.; Ezersky, V.; Makov, G.; Golan, Y. Synthesis and Properties of Nanocrystalline $\pi$-SnS - a New Cubic Phase of Tin Sulphide. RSC Adv. 2016, 6 (7), 5848-5855. https://doi.org/10.1039/C5RA23092F.

(80) Murnaghan, F. D. The Compressibility of Media under Extreme Pressures. Proc. N. A. S. 1944, 30, 244-247.

(81) Walsh, A.; Payne, D. J.; Egdell, R. G.; Watson, G. W. Stereochemistry of Post-Transition Metal Oxides: Revision of the Classical Lone Pair Model. Chem. Soc. Rev. 2011, 40 (9), 
4455-4463. https://doi.org/10.1039/c1cs15098g.

(82) Skelton, J. M.; Skelton, J. M.; Gunn, D. S. D.; Metz, S.; Metz, S.; Parker, S. C. Accuracy of Hybrid Functionals with Non-Self-Consistent Kohn-Sham Orbitals for Predicting the Properties of Semiconductors. J. Chem. Theory Comput. 2020, 16 (6), 3543-3557. https://doi.org/10.1021/acs.jctc.9b01218.

(83) Whittles, T. J.; Burton, L. A.; Skelton, J. M.; Walsh, A.; Veal, T. D.; Dhanak, V. R. Band Alignments, Valence Bands, and Core Levels in the Tin Sulfides SnS, SnS2, and Sn2S3: Experiment and Theory. Chem. Mater. 2016, 28 (11), 3718-3726. https://doi.org/10.1021/acs.chemmater.6b00397.

(84) Argaman, U.; Abutbul, R. E.; Golan, Y.; Makov, G. Phonon Band Gaps in the IV-VI $\begin{array}{llllllll}\text { Monochalcogenides. } & \text { Phys. } & \text { Rev. } & \text { B 2019, } & 100 & \text { (5), } & 1-12 .\end{array}$ https://doi.org/10.1103/PhysRevB.100.054104.

(85) Lanigan-Atkins, T.; Yang, S.; Niedziela, J. L.; Bansal, D.; May, A. F.; Puretzky, A. A.; Lin, J. Y. Y.; Pajerowski, D. M.; Hong, T.; Chi, S.; Ehlers, G.; Delaire, O. Extended Anharmonic Collapse of Phonon Dispersions in SnS and SnSe. Nat. Commun. 2020, 11 (1), 1-9. https://doi.org/10.1038/s41467-020-18121-4.

(86) Hellman, O.; Steneteg, P.; Abrikosov, I. A.; Simak, S. I. Temperature Dependent Effective Potential Method for Accurate Free Energy Calculations of Solids. Phys. Rev. B 2013, 87 (10), 104111. https://doi.org/10.1103/PhysRevB.87.104111.

(87) Monacelli, L.; Bianco, R.; Cherubini, M.; Calandra, M.; Errea, I.; Mauri, F. The Stochastic Self-Consistent Harmonic Approximation: Calculating Vibrational Properties of Materials with Full Quantum and Anharmonic Effects. 2021, 1-36.

(88) Souvatzis, P.; Eriksson, O.; Katsnelson, M. I.; Rudin, S. P. The Self-Consistent Ab Initio 
Lattice Dynamical Method. Comput. Mater. Sci. 2009, 44 (3), 888-894. https://doi.org/10.1016/j.commatsci.2008.06.016.

(89) Loa, I.; Husband, R. J.; Downie, R. A.; Popuri, S. R.; Bos, J. W. G. Structural Changes in Thermoelectric SnSe at High Pressures. J. Phys. Condens. Matter 2015, 27 (7). https://doi.org/10.1088/0953-8984/27/7/072202.

(90) Alptekin, S. Structural Phase Transition of SnSe under Uniaxial Stress and Hydrostatic Pressure: An Ab Initio Study. J. Mol. Model. 2011, 17 (11), 2989-2994. https://doi.org/10.1007/s00894-011-1019-2.

(91) Yu, H.; Dai, S.; Chen, Y. Enhanced Power Factor via the Control of Structural Phase Transition in SnSe. Sci. Rep. 2016, 6, 1-12. https://doi.org/10.1038/srep26193.

(92) Gashimzade, F. M.; Guseinova, D. A.; Jahangirli, Z. A.; Mekhtiev, B. G. Second-Order Phase Transition in a SnS Orthorhombic Crystal under Pressure. Phys. Solid State 2015, 57 (2), 378-380. https://doi.org/10.1134/S1063783415020110.

(93) Kantorovlch, L. N. Thermoelastic Properties. Phys. Rev. B 1995, 51 (6), 3520.

(94) Kantorovich, L. N. Thermoelastic Properties of Perfect Crystals with Nonprimitive Lattices. II. Application to $\mathrm{KCl}$ and $\mathrm{NaCl}$. Phys. Rev. $B$ 1995, 51 (6), 3535-3548. https://doi.org/10.1103/PhysRevB.51.3535.

(95) Blanco, M. A.; Francisco, E.; Luaña, V. GIBBS: Isothermal-Isobaric Thermodynamics of Solids from Energy Curves Using a Quasi-Harmonic Debye Model. Comput. Phys. Commun. 2004, 158 (1), 57-72. https://doi.org/10.1016/j.comphy.2003.12.001.

(96) Herbol, H. C.; Stevenson, J.; Clancy, P. Computational Implementation of Nudged Elastic Band, Rigid Rotation, and Corresponding Force Optimization. J. Chem. Theory Comput. 2017, 13 (7), 3250-3259. https://doi.org/10.1021/acs.jctc.7b00360. 
TOC Graphic:

We use first-principles quasi-harmonic lattice dynamics calculations to explore the phase space of the tin monochalcogenides $\mathrm{SnS}$ and $\mathrm{SnSe}$ as a function of temperature and pressure.

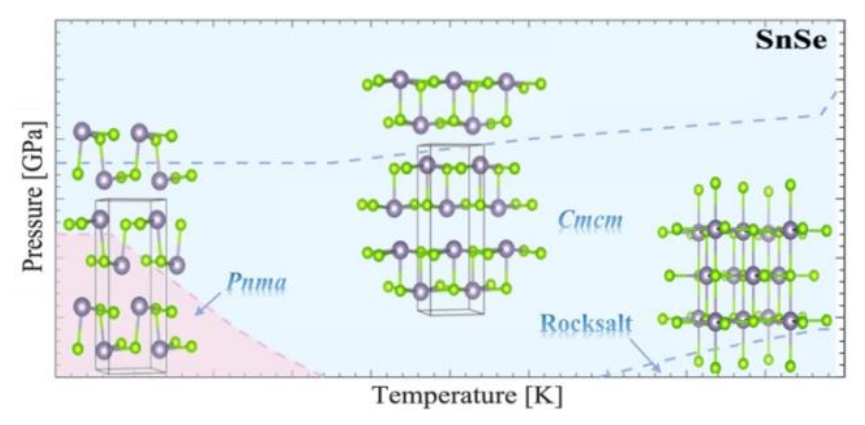




\title{
Phase Stability of the Tin Monochalcogenides SnS
}

\section{and SnSe: a Quasi-Harmonic Lattice-Dynamics}

\author{
Study
}

\section{Supporting Information}

\author{
Ioanna Pallikara $^{1}$ and Jonathan M. Skelton ${ }^{1 *}$
}

${ }^{1}$ Department of Chemistry, University of Manchester, Oxford Road, Manchester M13 9PL, UK

*E-Mail: jonathan.skelton@manchester.ac.uk 
(a) $\mathrm{SnS}$ (Pnma)

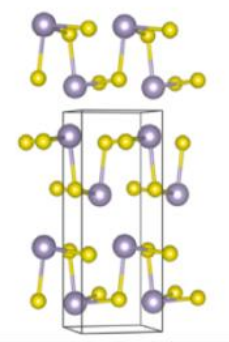

(b) $\mathrm{SnS}(\mathrm{Cmcm})$

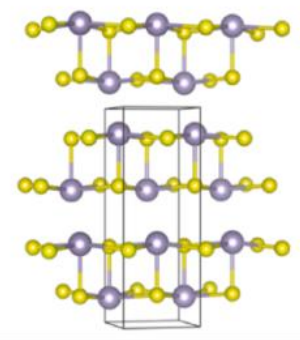

(c) $\mathrm{SnS}$ (Rocksalt)

(d) $\mathrm{SnS}$ ( $\pi$-cubic)

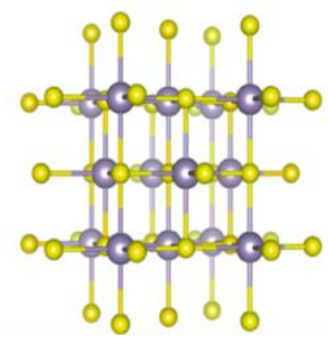

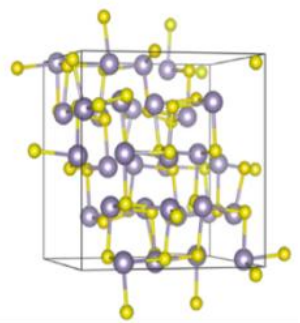

Figure S1. Optimised structures of Pnma, Cmcm, rocksalt and $\pi$-cubic SnS. These images were produced using the VESTA software. ${ }^{1}$
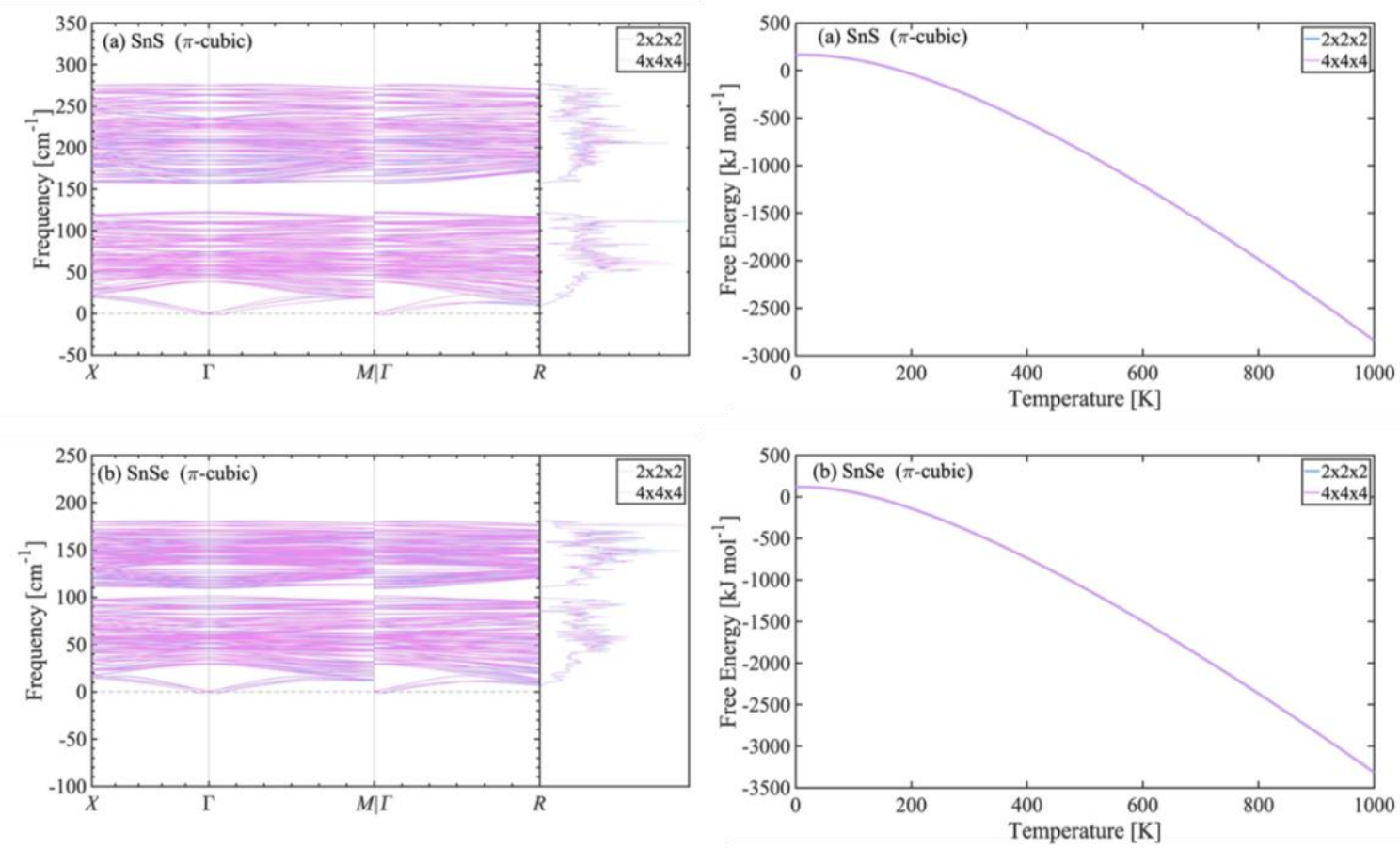

Figure S2. Phonon dispersion and density of states curves and vibrational Helmholtz free energy of the $\pi$-cubic phases of $\mathrm{SnS}$ (a) and $\mathrm{SnSe}$ (b) computed using two $\boldsymbol{k}$-point meshes with different numbers of subdivisions. Both calculations were performed on the equilibrium structure using the 64 -atom unit cell, so the equivalent $\boldsymbol{k}$-point meshes for the $2 \times 2 \times 2$ supercell used in the production calculations would be meshes with $1 \times 1 \times 1$ (i.e. the $\Gamma$ point) and $2 \times 2 \times 2$ subdivisions. 

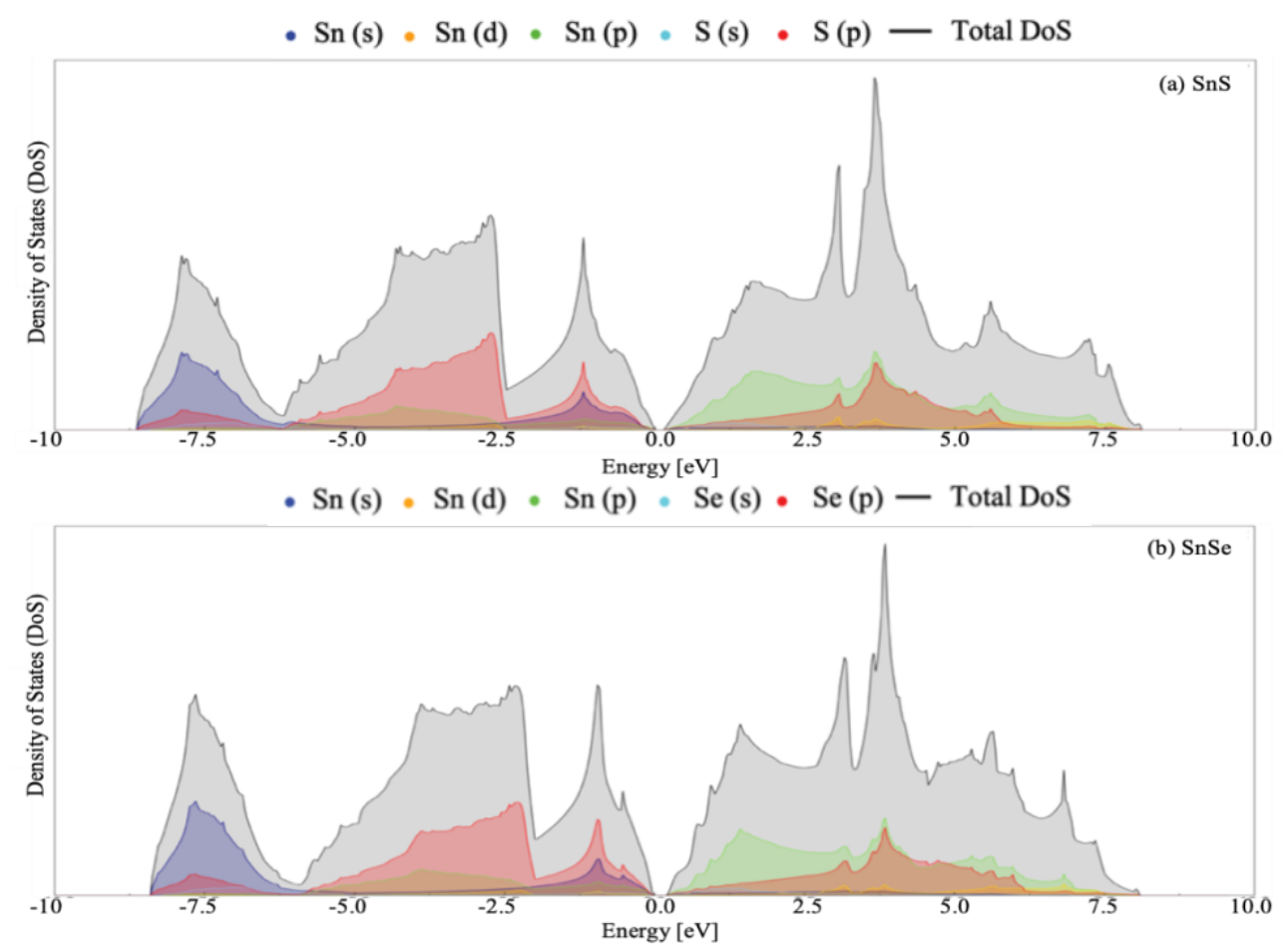

Figure S3. Calculated electronic atom- and orbital-projected density of states (DoS) curves for the rocksalt phases of (a) $\mathrm{SnS}$ and (b) SnSe. The states are colored as follows: $\mathrm{Sn}(\mathrm{s})$ - blue, $\mathrm{Sn}(\mathrm{d})$ - yellow, $\mathrm{Sn}(\mathrm{p})$ - green, $\mathrm{S} / \mathrm{Se}(\mathrm{s})$ - cyan, $\mathrm{S} / \mathrm{Se}(\mathrm{p})$ - red and total DoS - black. 
Table S1. Calculated electronic bandgaps of the four phases of SnS (top) and SnSe (bottom) obtained using the PBEsol + D3 functional, with experimental and theoretical data for comparison where available.

\begin{tabular}{|c|c|c|c|}
\hline \multicolumn{4}{|c|}{ Bandgaps: SnS [eV] } \\
\hline Phase & $\begin{array}{l}\text { PBEsol + D3 } \\
\text { (this work) }\end{array}$ & Experiments & Other DFT studies \\
\hline Rocksalt & 0.150 & - & $0.520^{2}$ \\
\hline Pnma & 0.579 & $1.330-1.600^{3}$ & $1.352^{4}, 1.800^{5}$ \\
\hline $\mathrm{Cmcm}$ & 0.549 & - & $1.400^{5}$ \\
\hline$\pi$-cubic & 1.130 (indirect) & $1.53^{6}$ & $1.740^{7}$ \\
\hline \multicolumn{4}{|c|}{ Bandgaps: SnSe [eV] } \\
\hline Phase & $\begin{array}{l}\text { PBEsol + D3 } \\
\text { (this work) }\end{array}$ & Experiments & Other DFT studies \\
\hline Rocksalt & 0.253 & - & $0.580^{2}$ \\
\hline Pnma & 0.423 & $0.890^{8}, 1.000-1.200^{9}$ & $0.790^{10}, 1.108,{ }^{4} 0.400^{11}$ \\
\hline $\mathrm{Cmcm}$ & 0.234 & $0.390^{8}$ & $0.050^{11}$ \\
\hline$\pi$-cubic & 0.856 (indirect) & $1.280^{12}$ & $0.799 / 1.011(\mathrm{LDA} / \mathrm{GGA})^{13}$ \\
\hline
\end{tabular}



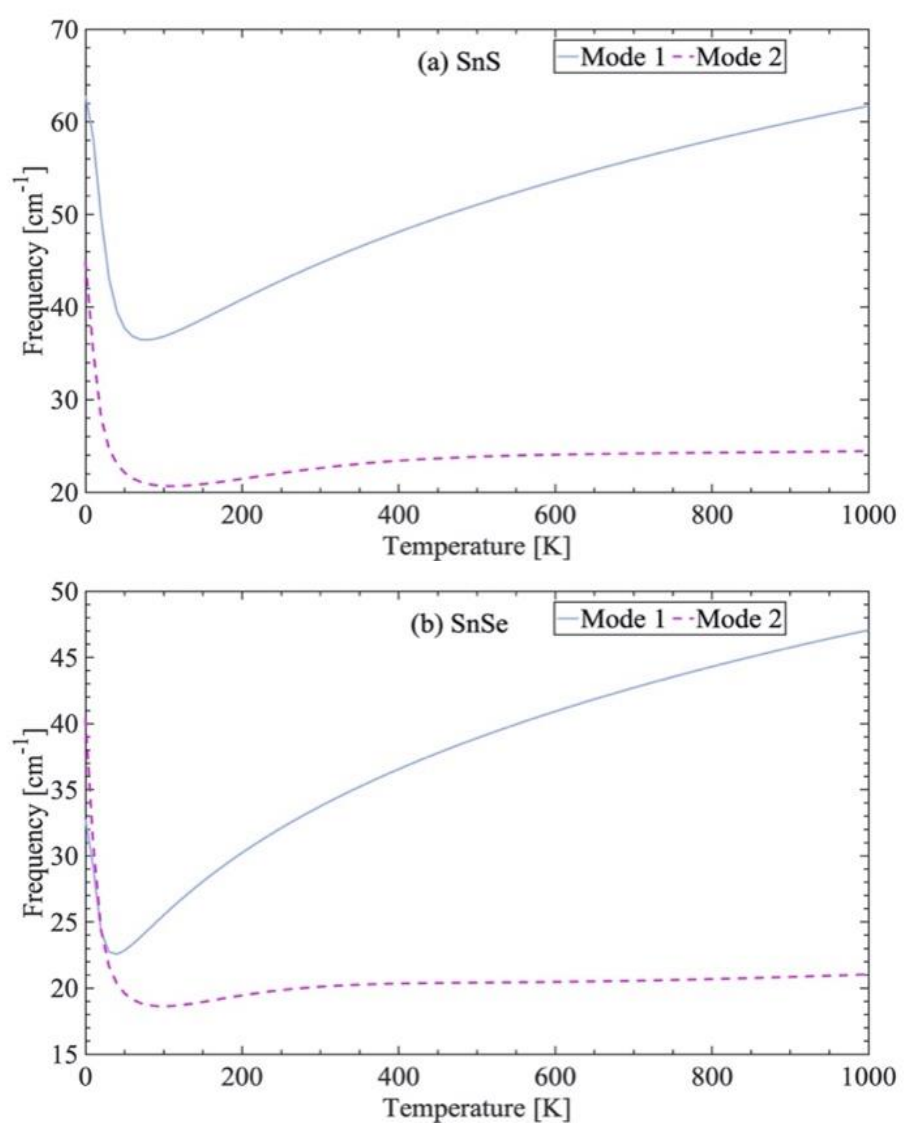

Figure S4. Effective renormalised frequencies of the two imaginary modes of $\mathrm{Cmcm} \mathrm{SnS} \mathrm{(a)}$ and $\mathrm{SnSe}(\mathrm{b})$ at the equilibrium volume, as a function of temperature, obtained using the method in Ref. ${ }^{14}$. 

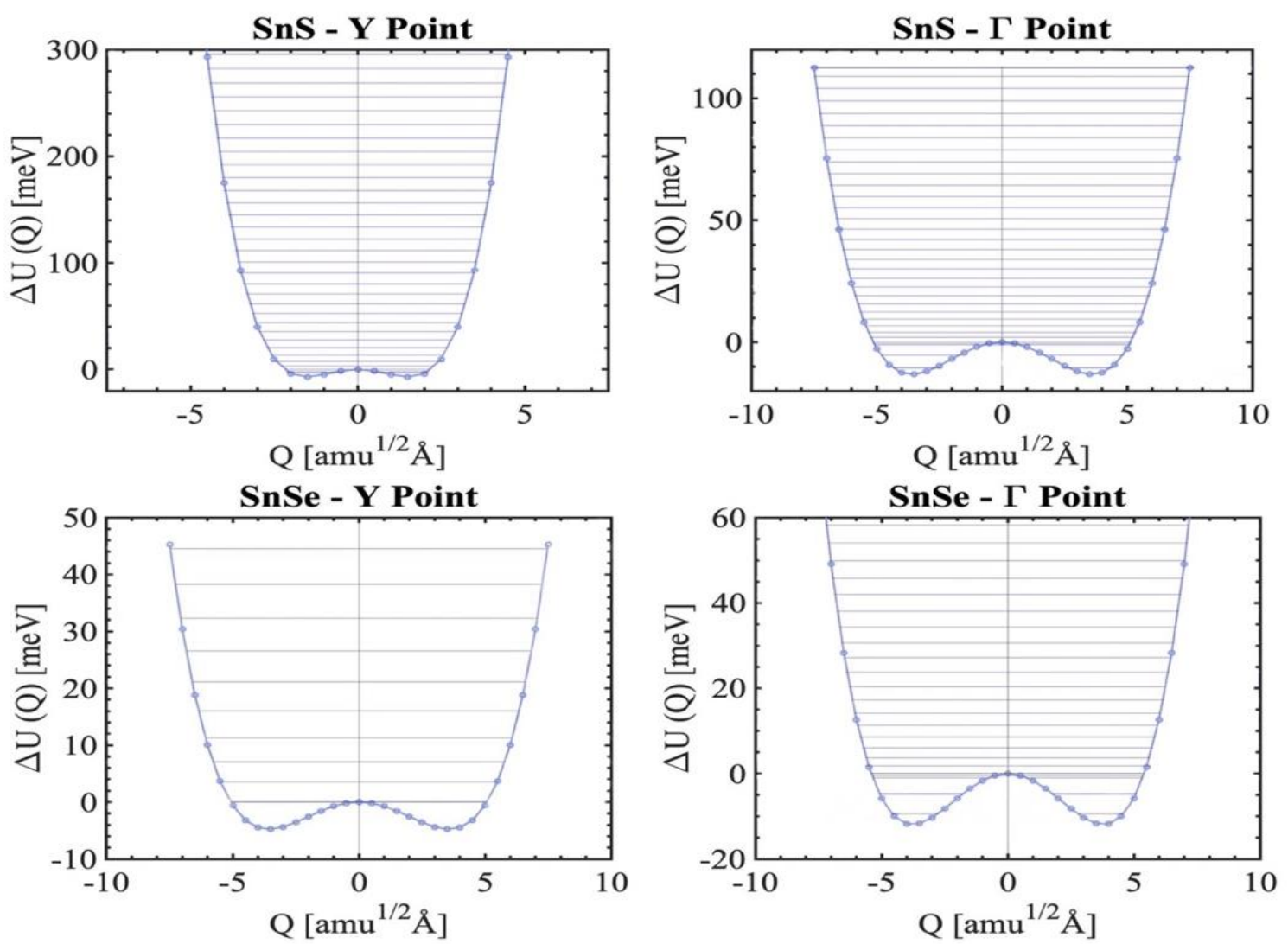

Figure S5. Anharmonic double-well potentials along the two principal imaginary modes at the $Y$ and $\Gamma$ wavevectors of $\mathrm{Cmcm} \mathrm{SnS}$ (top) and $\mathrm{SnSe}$ (bottom) at the equilibrium volume. The black lines inside the potentials show the eigenvalues obtained by solving a 1D Schrödinger equation for the potential. 

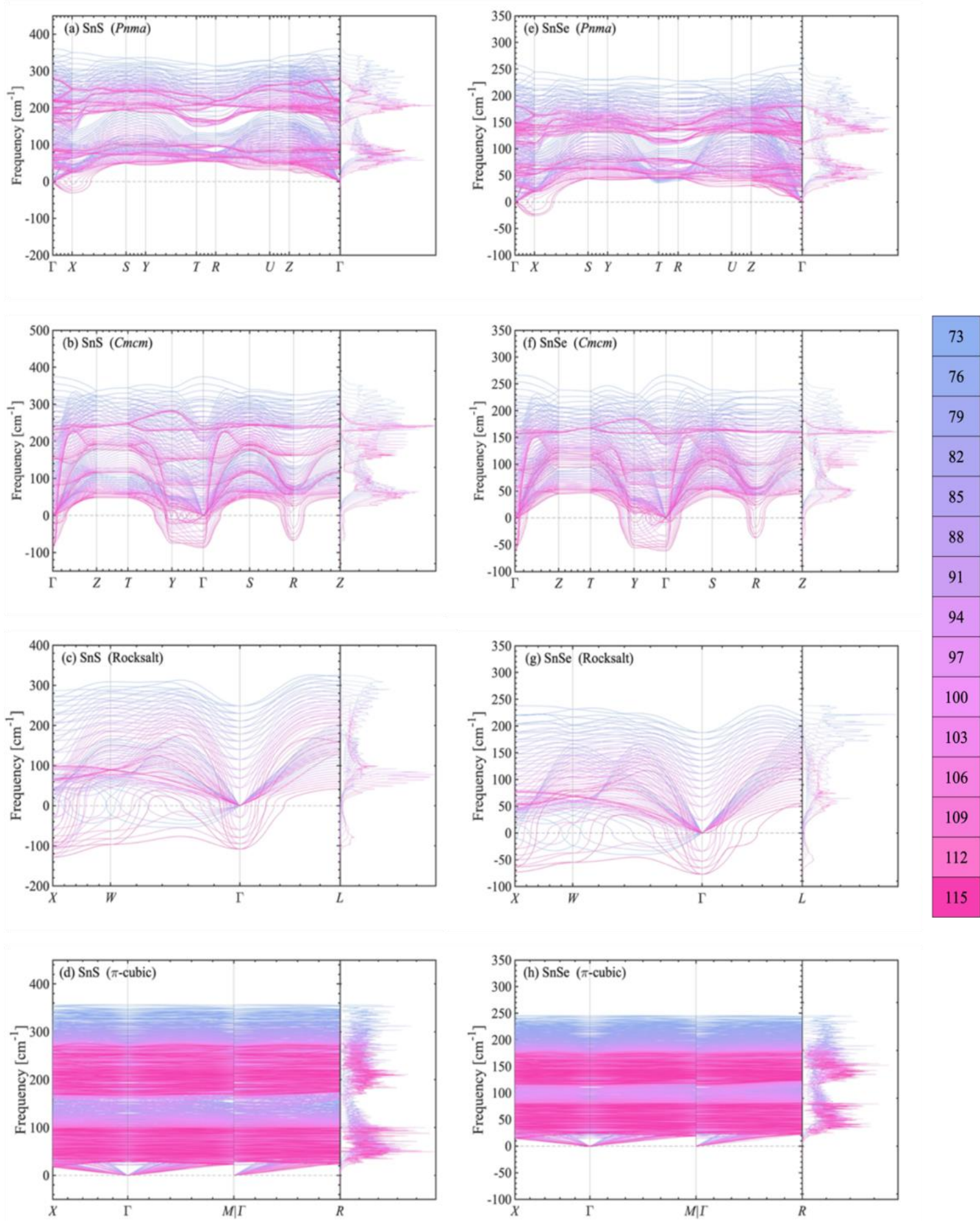

Figure S6. Phonon dispersion and density of states curves of SnS (left, (a)-(d)) and SnSe (right, (e)-(h)) under up to $27 \%$ volume compression and $15 \%$ expansion: (a)/(e) Pnma, (b)/(f) Cmcm, $(\mathrm{c}) /(\mathrm{g})$ rocksalt and $(\mathrm{d}) /(\mathrm{h}) \pi$-cubic. 

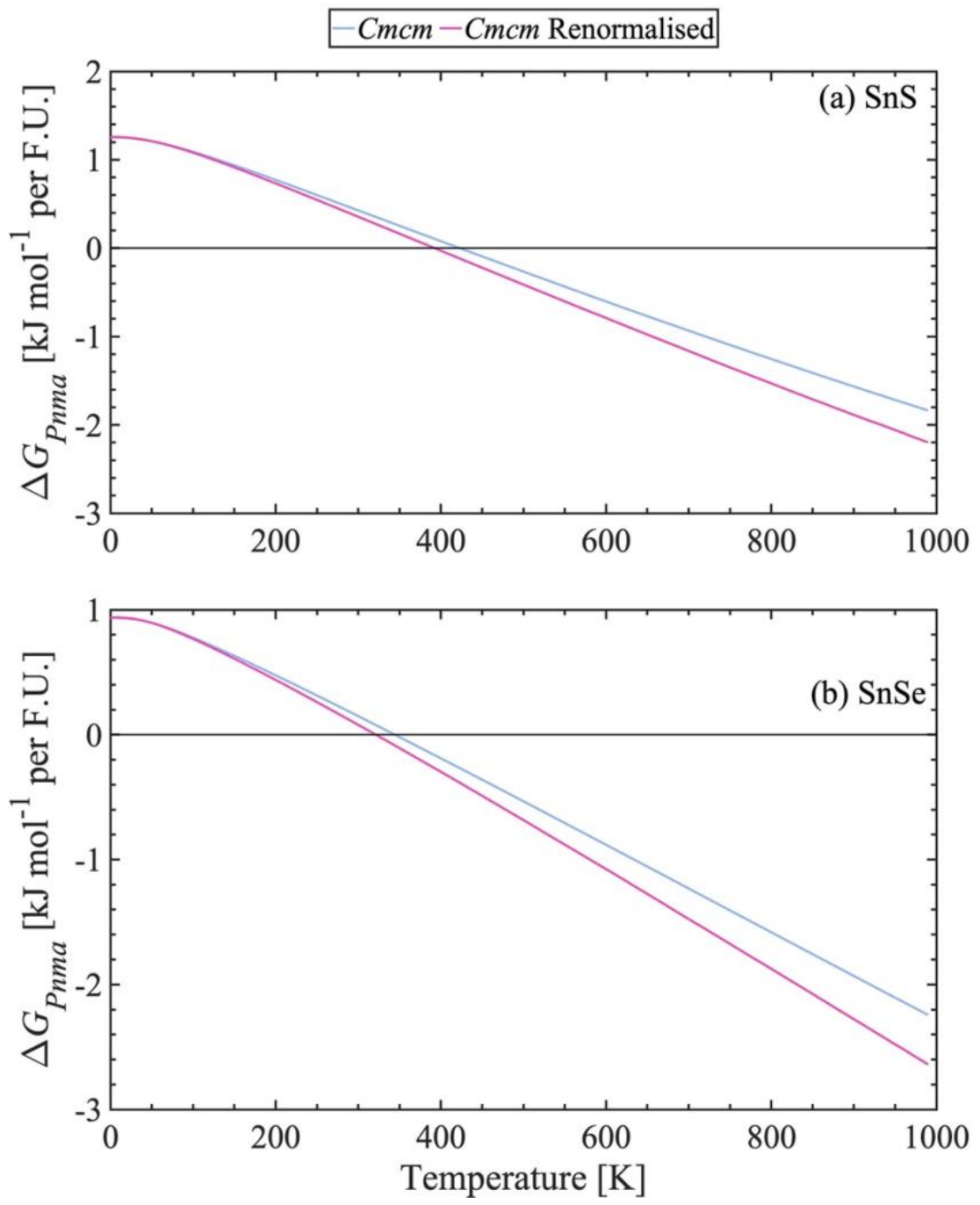

Figure S7. Gibbs energy differences $\Delta G_{P n m a}$ between the Pnma and Cmcm phases of (a) SnS and (b) SnSe before (blue) and after renormalization of the imaginary harmonic modes (pink). 
(a) $\mathrm{SnS}$

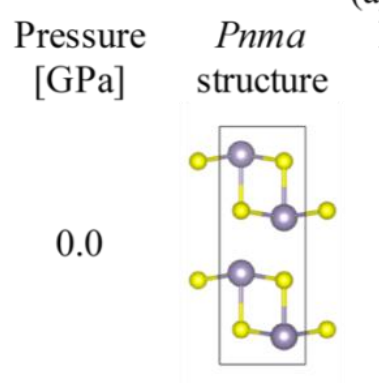

0.0

[GPa]

$\mathrm{Cmcm}$ structure

Pressure

[GPa]

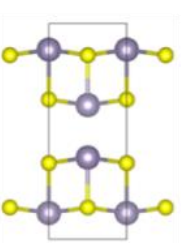

0.0
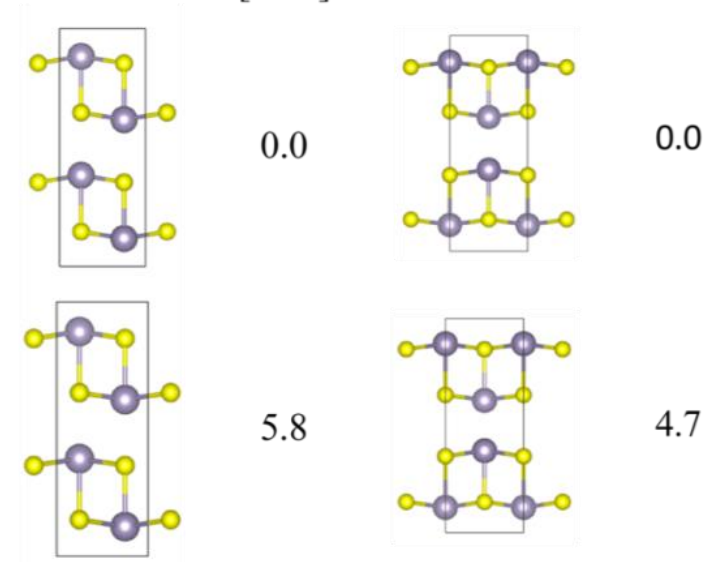

5.8

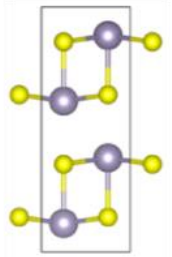

11.6

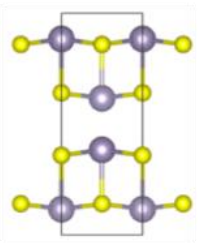

4.7

Pnma
structure

(b) $\mathrm{SnSe}$

Pressure

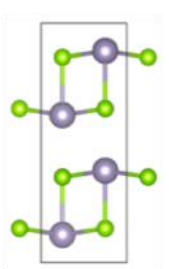

0.0

[GPa]

$\mathrm{Cmcm}$

structure

9.9

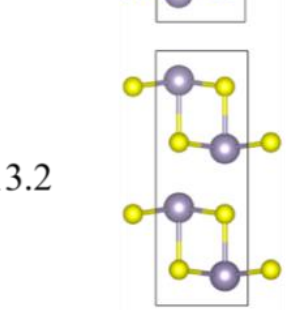

13.2
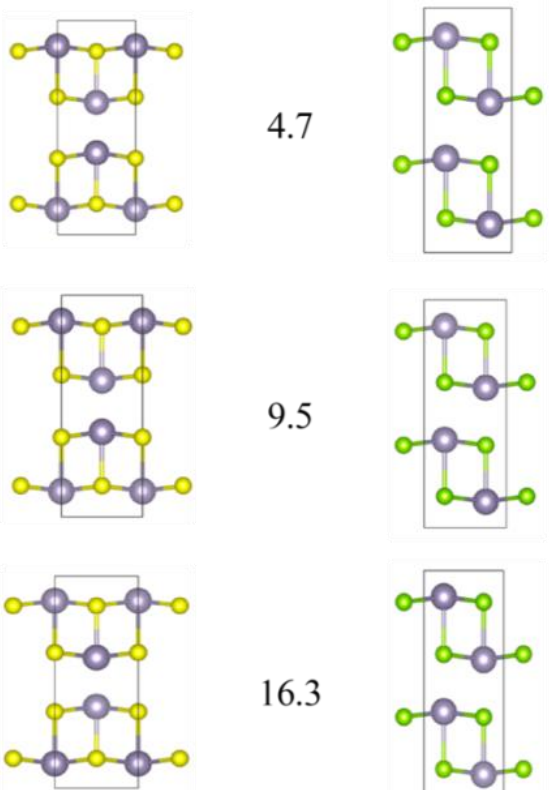

\section{4}

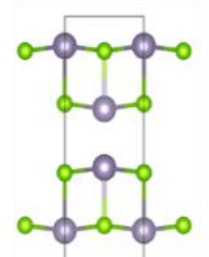

9.5
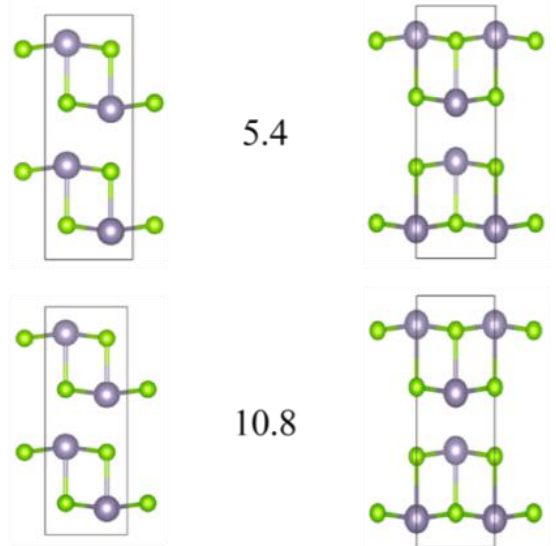

16.3

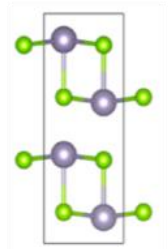

10.8

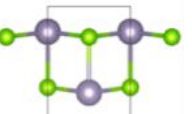

16.3

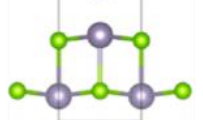

Figure S8. Optimized structures of Pnma and $\mathrm{Cmcm} \mathrm{SnS} \mathrm{(a)} \mathrm{and} \mathrm{SnSe}(\mathrm{b})$ at a range of applied pressures. The images were produced using the VESTA software. ${ }^{1}$ 
(a)

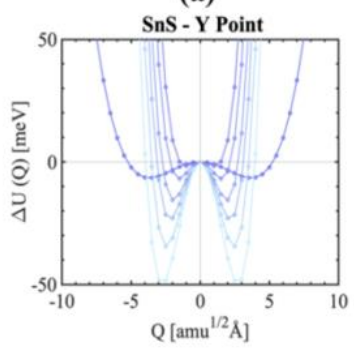

(c)

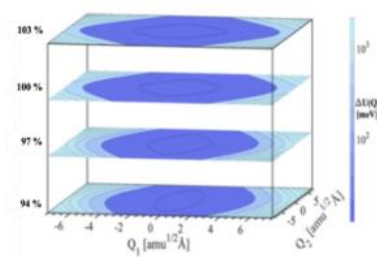

(b)

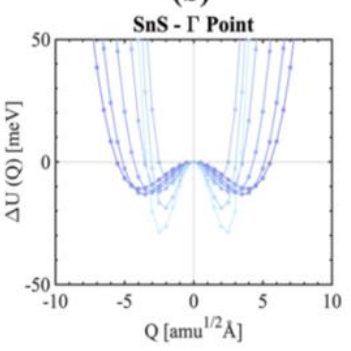

(d) (e)

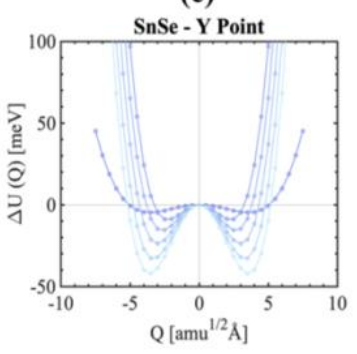

(g)

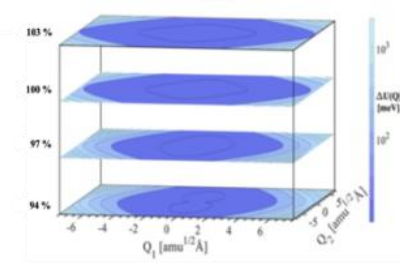

(f)

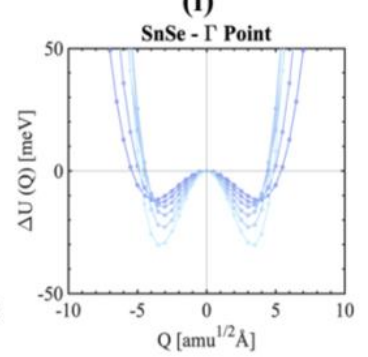

(h)
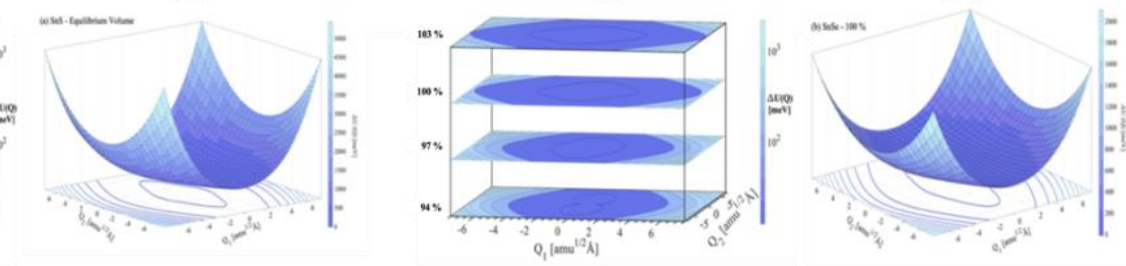

Figure S9. Potential-energy surface (PES) along the two principal imaginary modes at the $Y$ (a)/(e) and $\Gamma$ wavevectors (b)/(f) of (a)/(b) $\mathrm{Cmcm} \mathrm{SnS} \mathrm{and} \mathrm{(e)/(f)} \mathrm{Cmcm}$ SnSe, evaluated for a series of volumes including up to $6 \%$ compressions and $15 \%$ expansions. (c)/(g) Twodimensional PES spanned by both imaginary modes at four volumes ranging from $6 \%$ compression to $3 \%$ expansion. (d)/(h) Surface plot of the (PES) along the two principal imaginary modes in the equilibrium structures. 


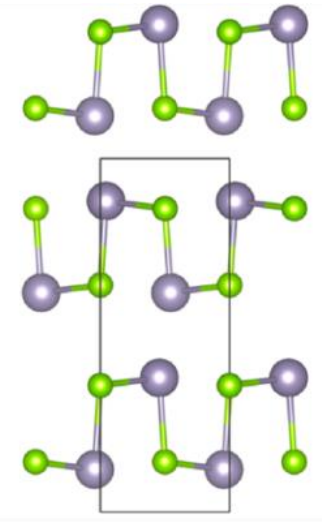

(a)
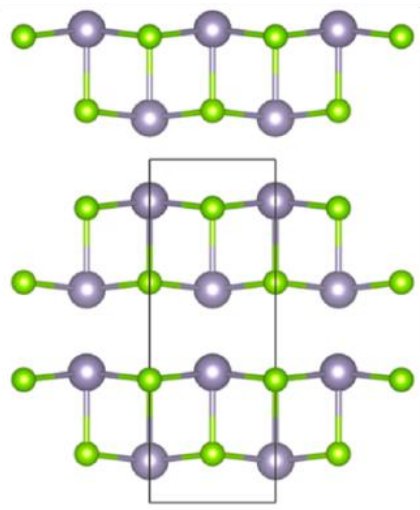

(b)

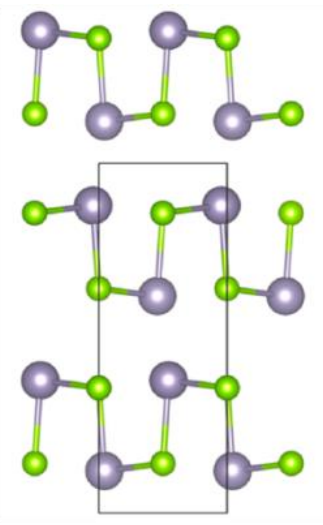

(c)

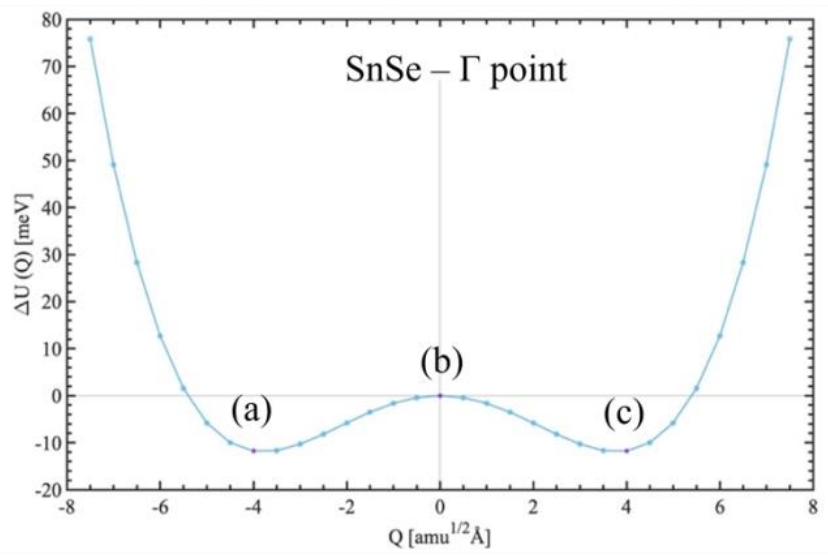

Figure S10. Structures of SnSe associated with the minima in the 1D potential-energy surface (PES) obtained by mapping the $\Gamma$-point imaginary mode in the equilibrium structure. (a) Minimum at $Q=-4 \mathrm{amu}^{1 / 2} \AA$. (b) Maximum at $Q=0$. (c) Minimum at $Q=+4 \mathrm{amu}^{1 / 2} \AA$. (a) and (c) correspond to the distorted Pnma phase, while (b) corresponds to the Cmcm average structure. 
(a) $\mathrm{SnS}$
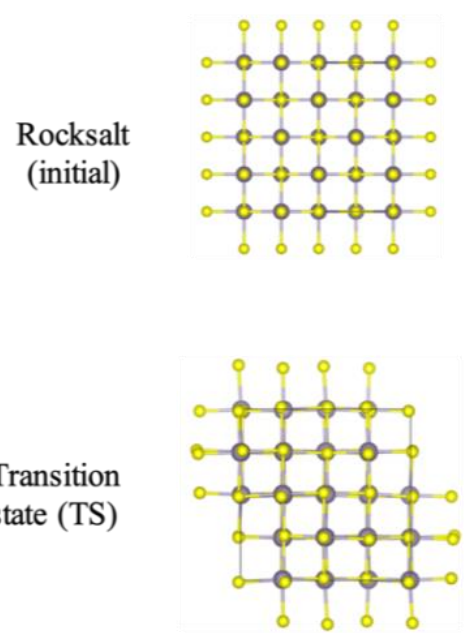

Transition state (TS)

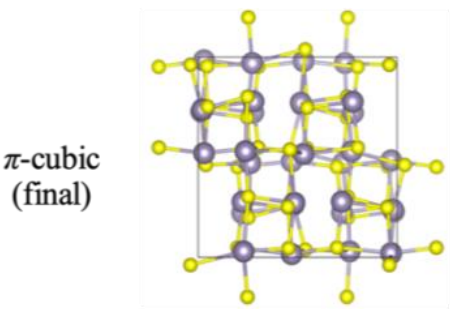

(b) $\mathrm{SnSe}$
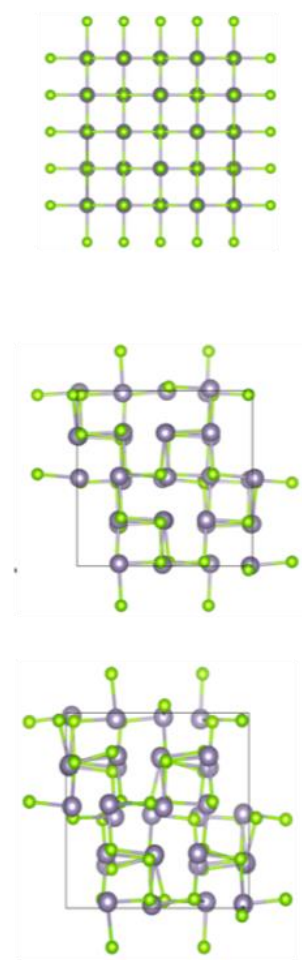

Figure S11. Structures of the initial (rocksalt), transition state (TS), and final ( $\pi$-cubic) structures of $\mathrm{SnS}$ (a) and $\mathrm{SnSe}(\mathrm{b})$ obtained from the climbing image nudged elastic band (CINEB) calculations. These images were produced by the VESTA software. ${ }^{1}$ 

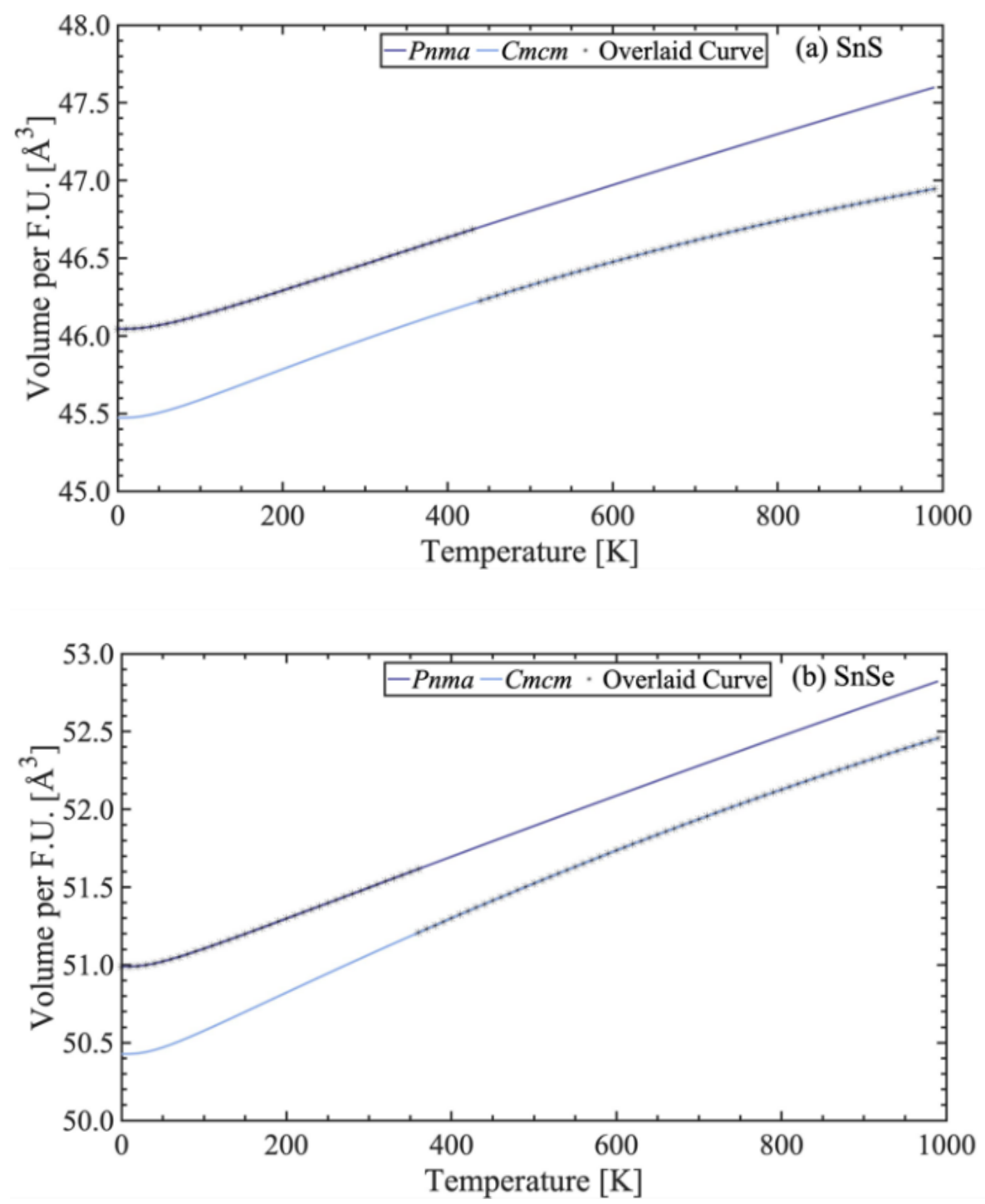

Figure S12. Calculated volume per formula unit as a function of temperature for the Pnma (purple) and Cmcm (light blue) phases of (a) SnS and (b) SnSe. The black stars track the volumes of the phases with the lowest-energy Gibbs free energy to highlight expected discontinuities in the volume at the phase transition. 

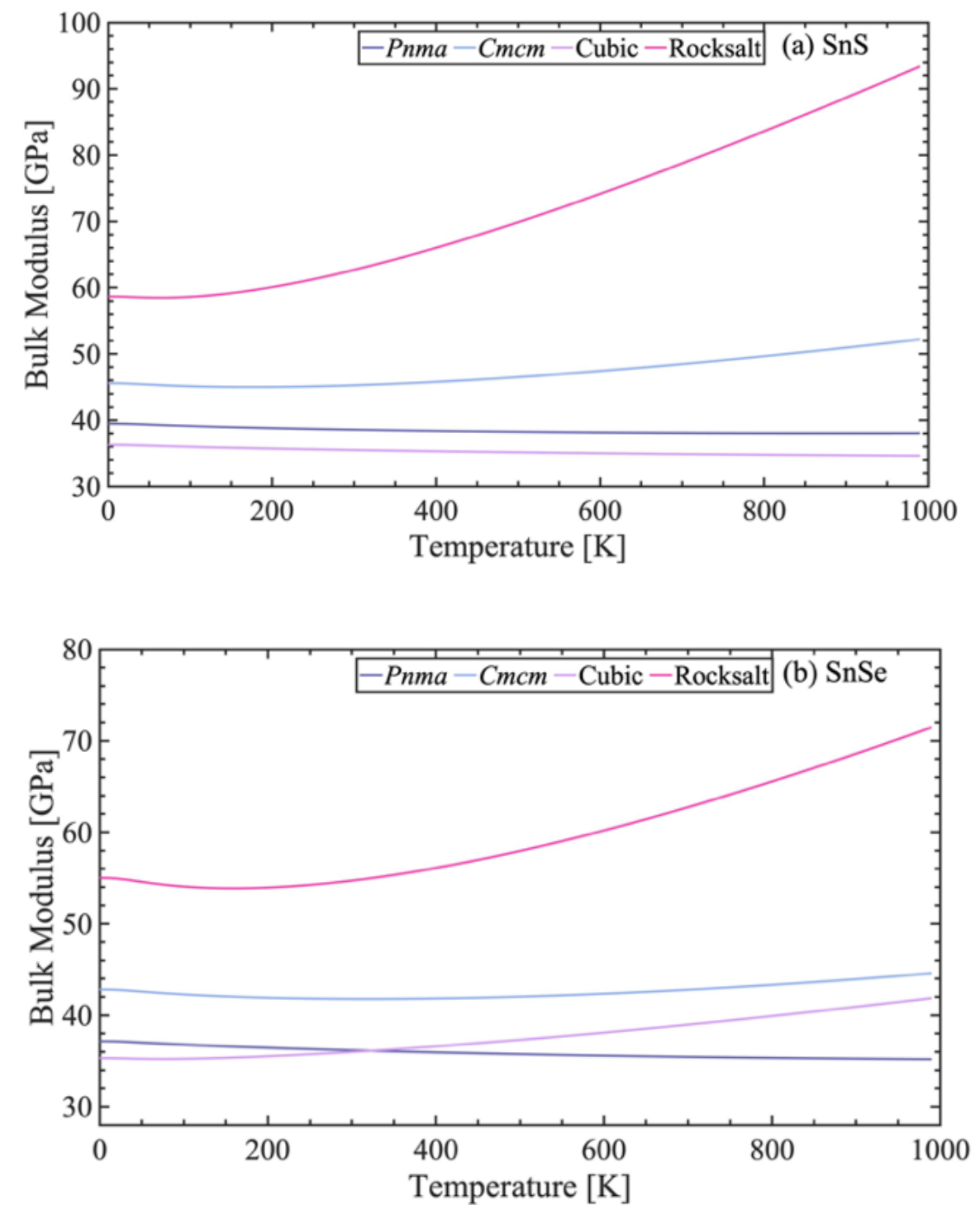

Figure S13. Calculated bulk modulus as a function of temperature for the Pnma (purple), Cmcm (light blue), $\pi$-cubic (purple) and rocksalt (pink) phases (a) SnS and (b) SnSe. 

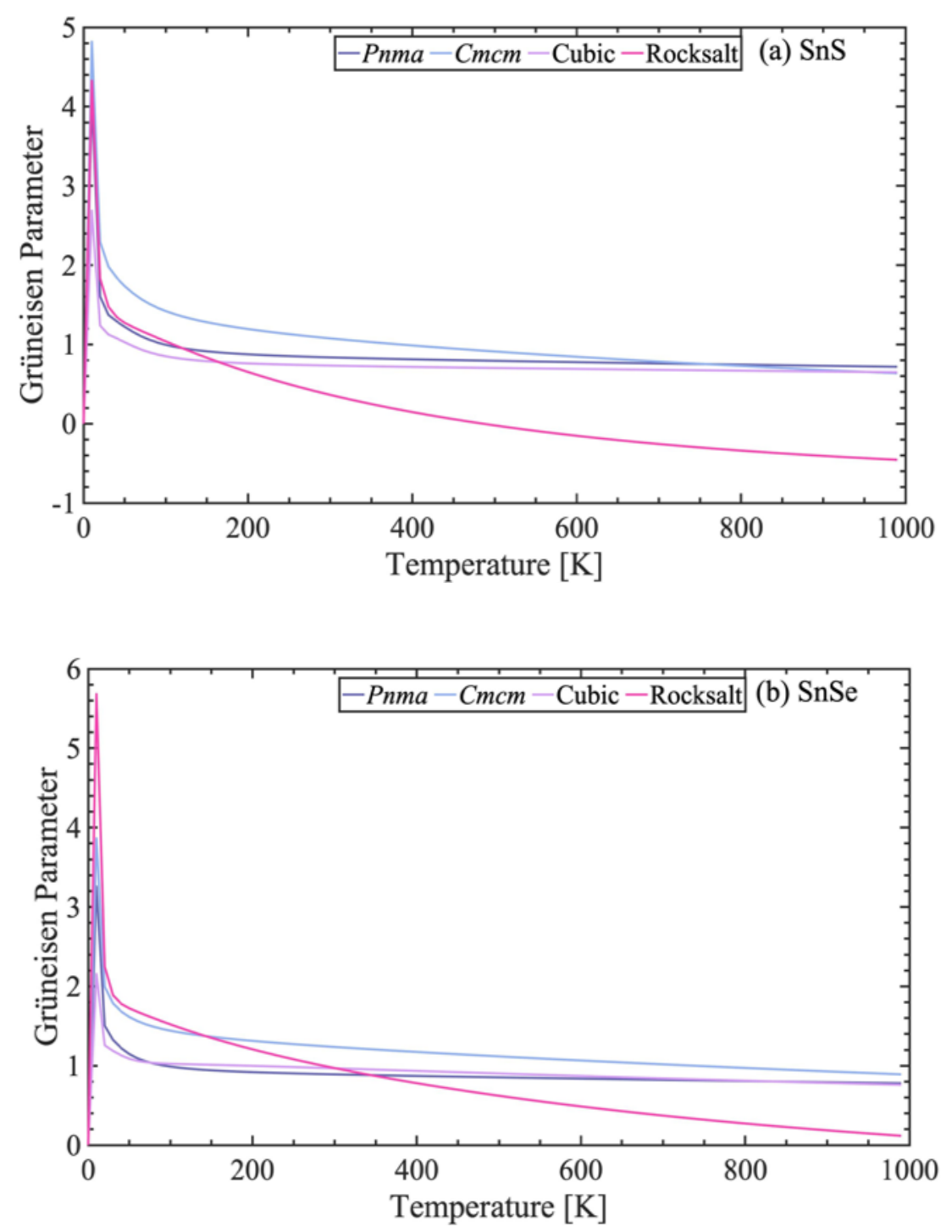

Figure S14. Calculated Grüneisen parameter as a function of temperature for the Pnma (purple), Cmcm (light blue), $\pi$-cubic (purple) and rocksalt (pink) phases (a) SnS and (b) SnSe. 


\section{REFERENCES:}

(1) Momma, K.; Izumi, F. VESTA3 for Three-Dimensional Visualization of Crystal, Volumetric and Morphology Data. J. Appl. Crystallogr. 2011, 44 (6), 1272-1276. https://doi.org/10.1107/S0021889811038970.

(2) Sun, Y.; Zhong, Z.; Shirakawa, T.; Franchini, C.; Li, D.; Li, Y.; Yunoki, S.; Chen, X. Q. Rocksalt SnS and SnSe: Native Topological Crystalline Insulators. Phys. Rev. B Condens. Matter Mater. Phys. 2013, $88 \quad$ (23), 235122-235122. https://doi.org/10.1103/PhysRevB.88.235122.

(3) Valiukonis, G.; Guseinova, D. A.; Krivaite, G.; Sileika, A. Optical Spectra and Energy Band Structure of Layer Type A(IV)B(VI) Compounds. Phys. Status Solidi 1986, 135 (2), 299-307. https://doi.org/https://doi.org/10.1002/pssb.2221350130.

(4) Walsh, A.; Watson, G. W. Influence of the Anion on Lone Pair Formation in Sn(II) Monochalcogenides: A DFT Study. J. Phys. Chem. B 2005, 109 (40), 18868-18875. https://doi.org/10.1021/jp051822r.

(5) Koteeswara Reddy, N.; Devika, M.; Gopal, E. S. R. Review on Tin (II) Sulfide (SnS) Material: Synthesis, Properties, and Applications. Crit. Rev. Solid State Mater. Sci. 2015, 40 (6), 359-398. https://doi.org/10.1080/10408436.2015.1053601.

(6) Abutbul, R. E.; Segev, E.; Zeiri, L.; Ezersky, V.; Makov, G.; Golan, Y. Synthesis and Properties of Nanocrystalline $\pi-\mathrm{SnS}$ - a New Cubic Phase of Tin Sulphide. RSC Adv. 2016, 6 (7), 5848-5855. https://doi.org/10.1039/C5RA23092F.

(7) Skelton, J. M.; Burton, L. A.; Oba, F.; Walsh, A. Metastable Cubic Tin Sulfide: A Novel Phonon-Stable Chiral Semiconductor. APL Mater. 2017, 5 (3). https://doi.org/10.1063/1.4977868.

(8) Zhao, L. D.; Lo, S. H.; Zhang, Y.; Sun, H.; Tan, G.; Uher, C.; Wolverton, C.; Dravid, V. P.; Kanatzidis, M. G. Ultralow Thermal Conductivity and High Thermoelectric Figure of Merit in SnSe Crystals. Nature 2014, 508 (7496), 373-377. 
https://doi.org/10.1038/nature13184.

(9) Murnaghan, F. D. The Compressibility of Media under Extreme Pressures. Proc. N. A. S. 1944, 30, 244-247.

(10) Yang, L. T.; Ding, L. P.; Shao, P.; Tiandong, Y. H.; Zhao, Z. L.; Zhang, F. H.; Lu, C. Influence of Pressure on Phase Transition, Electronic and Thermoelectric Properties of $\begin{array}{llllll}\text { SnSe. } & \text { J. } & \text { Alloys } & \text { Compd. } & \text { 2021, } & 853,\end{array}$ https://doi.org/10.1016/j.jallcom.2020.157362.

(11) Lu, Y.; Zheng, F. W.; Yang, Y.; Zhang, P.; Zhang, D. B. Phase Stabilities of Cmcm and Pnma SnSe Studied by Phonon Quasiparticle Approach. Phys. Rev. B 2019, 100 (5). https://doi.org/10.1103/PhysRevB.100.054304.

(12) Abutbul, R. E.; Segev, E.; Samuha, S.; Zeiri, L.; Ezersky, V.; Makov, G.; Golan, Y. A New Nanocrystalline Binary Phase: Synthesis and Properties of Cubic Tin Monoselenide. CrystEngComm 2016, $18 \quad$ (11), 1918-1923. https://doi.org/10.1039/c5ce02437d.

(13) Rehman, S. U.; Butt, F. K.; Hayat, F.; Ul Haq, B.; Tariq, Z.; Aleem, F.; Li, C. An Insight into a Novel Cubic Phase SnSe for Prospective Applications in Optoelectronics and Clean Energy Devices. J. Alloys Compd. 2018, 733, 22-32. https://doi.org/10.1016/j.jallcom.2017.10.192.

(14) Skelton, J. M.; Burton, L. A.; Parker, S. C.; Walsh, A.; Kim, C.-E.; Soon, A.; Buckeridge, J.; Sokol, A. A.; Catlow, C. R. A.; Togo, A.; Tanaka, I. Anharmonicity in the High-Temperature Cmcm Phase of SnSe: Soft Modes and Three-Phonon $\begin{array}{lllllll}\text { Interactions. } & \text { Phys. } & \text { Rev. Lett. 2016, } & 117 & \text { (7), } & 075502 .\end{array}$ https://doi.org/10.1103/PhysRevLett.117.075502. 